\title{
Stereoselective Olefin Isomerization Leading to Asymmetric Quaternary Carbon Construction
}

\author{
Kan Wang, Christopher J. Bungard, and Scott G. Nelson* \\ Department of Chemistry, University of Pittsburgh, Pittsburgh, Pennsylvania 15260 \\ e-mail:sgnelson@pitt.edu
}

\section{TABLE OF CONTENTS}

General Procedures A and B: Preparation of di(allyl) ethers 7a-h, 14, and 16a/b

Compounds 7a-h, 14, 16a/b

General Procedure C: ICR reactions of di(allyl) ethers

Compounds 8a-h, 15, 17, 18a/b

$\mathrm{X}$-ray structure determination for $\mathbf{8 a}$-semicarbazide

${ }^{1} \mathrm{H}$ and ${ }^{13} \mathrm{C}$ Spectra for compounds 7, 8, 14-18
S 2

S 2-6

S 6

S 6-12

S 13

S $14-68$

General Information: Unless otherwise stated, all reactions were performed in dry glassware under an atmosphere of oxygen-free nitrogen using standard inert atmosphere techniques for the manipulation of solvents and reagents. Anhydrous solvents were obtained by passage through successive alumina- and Q5-packed columns on a solvent purification system. Acetone was distilled from Drierite and stored under nitrogen. Sodium hydride (60\% dispersion in mineral oil) was washed with hexanes $(3 \times)$ and dried under vacuum before use. 1,2-Dichloroethane (1,2-DCE), crotyl bromide, allyl bromide, and allyl acetate were distilled from $\mathrm{CaH}_{2}$. $\left[\left({ }^{C} \mathrm{C}_{8} \mathrm{H}_{14}\right)_{2} \mathrm{IrCl}\right]_{2}{ }^{1}$ and $\mathrm{PCy}_{3}$ were stored and weighed out in a nitrogen-filled glove box. The alkylating agents used in preparing di(allyl) ethers $\mathbf{7 a - e}, \mathbf{g}, \mathbf{h}, \mathbf{1 4}, \mathbf{1 6} \mathbf{a} / \mathbf{b}$ were prepared according to the literature procedures and ensuing alcohol $\rightarrow$ bromide conversion $\left(\mathrm{PBr}_{3}\right){ }^{2}$ Temperatures for the catalyzed Claisen rearrangements were controlled using an Ika ${ }^{\circledR}$ Werke hotplate/stirrer. Proton NMR spectra were recorded on a Bruker Avance-300 (300 $\mathrm{MHz}$ ) spectrometer with chemical shifts reported relative to residual $\mathrm{CHCl}_{3}(7.27 \mathrm{ppm})$ for ${ }^{1} \mathrm{H}$ and $\mathrm{CDCl}_{3}(77.00$ ppm) for ${ }^{13} \mathrm{C}$ NMR spectra. Mass spectra were obtained on a VG-7070 or Fisons Autospec high-resolution magnetic sector mass spectrometer. Analytical thin layer chromatography was performed on EM Reagent 0.25 $\mathrm{mm}$ silica gel $60-\mathrm{F}$ plates. Flash chromatography was performed as previously described on Iatrobeads 6RS-8060 (pH 7 silica gel), purchased from Shell-USA, or EM silica gel 60 (230-240 mesh). ${ }^{3} \quad$ Analytical high performance liquid chromatography (HPLC) was performed using a variable wavelength UV detector (deuterium lamp, 190-600 $\mathrm{nm})$, using Daicel Chiracel ${ }^{\mathrm{TM}}$ columns $(250 \times 4.6 \mathrm{~mm})$. HPLC-grade isopropanol and hexanes were used as the eluting solvents.

(1) Onderdelinden, A. L.; van der Ent, A. Inorg. Chim. Acta 1972, 6, 420.

(2) (a) Duboudin, J. G.; Jousseaume, B. J. Organomet. Chem. 1979, 168, 1-11. (b) Duboudin, J. G.; Jousseaume, B. J. Organomet. Chem. 1979, 168, 227-232.

(3) Still, W. C.; Kahn, M.; Mitra, A. J. Org. Chem. 1978, 43, 2923-2925. 


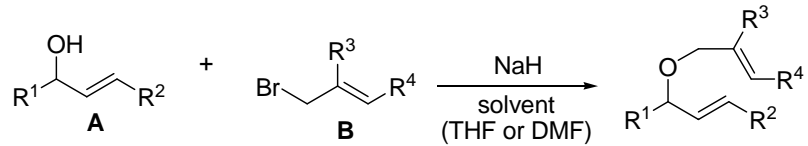

General Procedure A for preparing di(allyl) ethers 7a-h, 14, 16a/b (THF solvent): A solution of the allylic alcohol $\mathrm{A}$ (1.0 equiv) in anhydrous THF $(0.5 \mathrm{M})$ was added to a

suspension of pre-washed $\mathrm{NaH}(2.0$ equiv) in anhydrous THF $(1.0 \mathrm{M})$. The resulting suspension $(0.25 \mathrm{M})$ was heated at reflux for 30 min whereupon the allylic bromide $\mathbf{B}$ (1.0 equiv) was added and the resulting suspension heated at reflux for a further $2 \mathrm{~h}$. The reaction mixture was then cooled to ambient temperature and poured onto ice. The THF was evaporated under reduced pressure and the aqueous residue was extracted with $\mathrm{Et}_{2} \mathrm{O}(3 \times)$ and the combined organic extracts were dried over anhydrous $\mathrm{MgSO}_{4}$. The solvent was evaporated in vacuo and the residue was purified as indicated.

General Procedure A for preparing di(allyl) ethers (DMF solvent): Sodium hydride (2.5 equiv, 60\% suspension in mineral oil) was added to a $0{ }^{\circ} \mathrm{C}$ solution of alcohol $\mathbf{A}$ (1.0 equiv) in DMF (0.5 M) and the reaction was stirred 30 min. Allylic bromide $\mathbf{B}$ (1.0 equiv) was added and the resulting suspension was warmed to ambient temperature and stirred for $3 \mathrm{~h}$. Water was added slowly to quench the reaction and the resulting mixture was extracted with $\mathrm{Et}_{2} \mathrm{O}(3 \times)$. The combined organic extracts were dried over anhydrous $\mathrm{MgSO}_{4}$, the solvent was evaporated in vacuo, and the residue was purified as indicated.

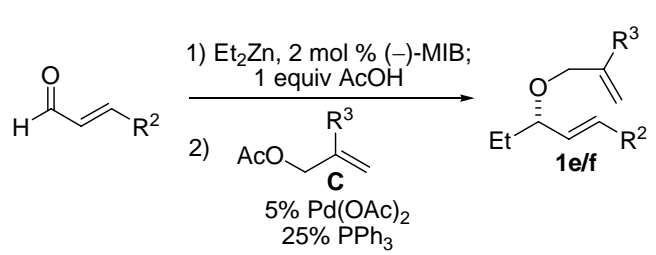

General Procedure B: One-pot preparation of di(allyl) ethers $(7 \mathrm{~d} / \mathrm{e})$ : To a $\quad 0 \quad{ }^{\circ} \mathrm{C} \quad$ solution of 1,7,7-trimethyl-3-morpholinobicyclo[2.2.1]heptan-2-ol [(-)-MIB] ${ }^{4}(2 \mathrm{~mol} \%, 0.02 \mathrm{mmol})$ in $2 \mathrm{~mL}$ of toluene ${ }^{5}$ was added $\mathrm{Et}_{2} \mathrm{Zn}(1.0 \mathrm{M}$ in hexanes, 1.05 equiv, $1.05 \mathrm{~mL})$ via syringe. A solution of aldehyde $(1.0 \mathrm{mmol})$ in $1 \mathrm{~mL}$ of toluene (or pentane) was added slowly ( $0.25 \mathrm{M}$ final concentration in aldehyde) and the reaction mixture was stirred at $0{ }^{\circ} \mathrm{C}$ until the reaction was complete as monitored by TLC $(\sim 6 \mathrm{~h})$. A solution of acetic acid (1.05 equiv) in $2 \mathrm{~mL}$ of THF was added dropwise and the resulting solution was stirred 10 min. A solution of $\mathrm{Pd}(\mathrm{OAc})_{2}(5 \mathrm{~mol} \%), \mathrm{PPh}_{3}$ (25 mol \%) and substituted allylic acetate C (1.0 equiv.) in $2 \mathrm{~mL}$ of THF (0.13 M final concentration) was added and the reaction was heated at $60{ }^{\circ} \mathrm{C}$ for $12 \mathrm{~h}$. The reaction mixture was cooled to ambient temperature, the solvent was evaporated, and the resulting crude product mixture was purified as indicated. Enantioselectivity for the $\mathrm{Et}_{2} \mathrm{Zn}$ addition was determined for the allylic alcohol obtained by quenching the $\mathrm{Et}_{2} \mathrm{Zn}$ addition reaction with saturated aqueous $\mathrm{NH}_{4} \mathrm{Cl}$ solution and extracting the resulting mixture with $\mathrm{Et}_{2} \mathrm{O}(3 \times)$. The combined organic extracts were dried $\left(\mathrm{MgSO}_{4}\right)$, concentrated, and purified as indicated.

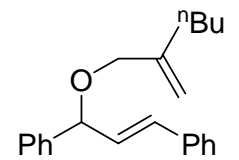

(E)-[3-(2-Methylenehexyloxy)prop-1-ene-1,3-diyl]dibenzene (7a): Two drops of concentrated $\mathrm{H}_{2} \mathrm{SO}_{4}$ were added to a solution of $0.489 \mathrm{~g}$ of (E)-1,3-diphenyl-2-propen-1-ol ${ }^{6}(2.33 \mathrm{mmol})$ and $0.474 \mathrm{~mL}$ of 2-methylenehexan-1-ol ${ }^{7}(3.49 \mathrm{mmol})$ in anhydrous THF $(1 \mathrm{~mL})$. The resulting solution was stirred for $4 \mathrm{~h}$ at ambient temperature whereupon $\mathrm{H}_{2} \mathrm{O}(10 \mathrm{~mL})$ was added and the resulting mixture was extracted with $\mathrm{Et}_{2} \mathrm{O}(3 \times)$. The combined organic extracts were washed with brine, dried $\left(\mathrm{MgSO}_{4}\right)$ and filtered. The solvent was evaporated and the product was purified by MPLC (0-5\% EtOAc in hexanes) to afford $0.490 \mathrm{~g}$

(4) Nugent, W. A. J. Chem. Soc., Chem. Commun. 1999, 1369-1370.

(5) Pentane was substituted for toluene as the reaction solvent as noted in the individual experimentals.

(6) Stoner, E, J.; Cothron, D. A.; Balmer, M. K.; Roden, B. A. Tetrahedron 1995, 51, 11043.

(7) Yadav, J. S.; Joshi, B.; Sahasrabubhe, A. Synth. Comm. 1985, 15, 797-805. 
(69\%) of the title compound as colorless oil. ${ }^{1} \mathrm{H} \mathrm{NMR}\left(300 \mathrm{MHz}, \mathrm{CDCl}_{3}\right): \delta 0.90(\mathrm{t}, \mathrm{J}=7.2 \mathrm{~Hz}, 3 \mathrm{H}), 1.26-1.29(\mathrm{~m}$, 4H), 2.09 (m, 2H), 3.97 (s, 2H), $4.93(\mathrm{~s}, 1 \mathrm{H}), 4.96(\mathrm{~d}, \mathrm{~J}=7.1 \mathrm{~Hz}, 1 \mathrm{H}), 5.06(\mathrm{~s}, 1 \mathrm{H}), 6.30$ (dd, J = 16, 7.0 Hz, 1H), $6.62(\mathrm{~d}, \mathrm{~J}=16 \mathrm{~Hz}, 1 \mathrm{H}), 7.20-7.45(\mathrm{~m}, 10 \mathrm{H}) ;{ }^{13} \mathrm{C} \mathrm{NMR}\left(75 \mathrm{MHz}, \mathrm{CDCl}_{3}\right): \delta 13.9,22.4,29.8,32.9,71.0,81.3,111.1$, 126.5, 126.8, 127.5, 127.6, 128.4, 128.5, 130.3, 131.2, 136.6, 141.2, 146.3; MS (EI, $70 \mathrm{eV}): \mathrm{m} / \mathrm{z} 306\left(\mathrm{M}^{+}\right), 288,262$, 206, 193, 181, 165, 158, 143, 129, 115, 105, 91, 77, 65; HRMS m/z calcd for $\mathrm{C}_{22} \mathrm{H}_{26} \mathrm{O}\left(\mathrm{M}^{+}\right)$: 306.1984; found: 306.1984 .

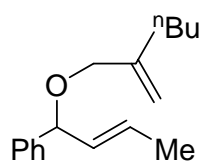

(E)-[1-(2-Methylenehexyloxy)but-2-enyl]benzene (7b): General Procedure A was followed employing $1.00 \mathrm{~g}$ of (E)-1-phenylbut-2-en-1-ol ${ }^{3}(6.75 \mathrm{mmol})$ in THF. Purifying the crude product mixture by flash chromatography (flushing with hexanes $(400 \mathrm{~mL})$ then eluting with $2 \%$ EtOAc/hexanes) afforded $1.57 \mathrm{~g}(95 \%)$ of the title compound as colorless oil. ${ }^{1} \mathrm{H}$ NMR $(300 \mathrm{MHz}$, $\left.\mathrm{CDCl}_{3}\right): \delta 0.89(\mathrm{~m}, 3 \mathrm{H}), 1.25-1.46(\mathrm{~m}, 4 \mathrm{H}), 1.71(\mathrm{~d}, \mathrm{~J}=5.7 \mathrm{~Hz}, 3 \mathrm{H}), 2.06(\mathrm{~m}, 2 \mathrm{H}), 3.88(\mathrm{~s}, 2 \mathrm{H}), 4.72(\mathrm{~d}, \mathrm{~J}=6.7 \mathrm{~Hz}$, $1 \mathrm{H}), 4.89(\mathrm{~s}, 1 \mathrm{H}), 5.01(\mathrm{~s}, 1 \mathrm{H}), 5.59(\mathrm{ddd}, \mathrm{J}=15,6.6,0.9 \mathrm{~Hz}, 1 \mathrm{H}), 5.69(\mathrm{dq}, \mathrm{J}=15,6.2 \mathrm{~Hz}, 1 \mathrm{H}), 7.22-7.35(\mathrm{~m}, 5 \mathrm{H})$; ${ }^{13} \mathrm{C}$ NMR $\left(75 \mathrm{MHz}, \mathrm{CDCl}_{3}\right): \delta 13.8,17.6,22.4,32.9,70.7,81.4,110.9,126.7,127.2,127.8,128.2,132.3,132.4$, 141.8, 146.4; MS (EI, $70 \mathrm{eV}): \mathrm{m} / \mathrm{z} 244\left(\mathrm{M}^{+}\right), 229,147,131,119,115,105,91,77,55 ;$ HRMS $\mathrm{m} / \mathrm{z}$ calcd for $\mathrm{C}_{17} \mathrm{H}_{24} \mathrm{O}$ $\left(\mathrm{M}^{+}\right)$: 244.1827; found: 244.1821 .

1-(2-\{[(E)-Oct-2-en-4-yloxy]methyl $\}$ allyl)benzene (7c): General Procedure A was followed
employing (E)-oct-2-en-4-ol $(256 \mathrm{mg}, 2.0 \mathrm{mmol})$ and 1-[2-(bromomethyl)allyl]benzene ${ }^{8}(630 \mathrm{mg}$, EtOAc in hexanes) to yield $397 \mathrm{mg}(77 \%)$ of the title compound as a light yellow oil. ${ }^{1} \mathrm{H} \mathrm{NMR}(300 \mathrm{MHz}$, $\left.\mathrm{CDCl}_{3}\right): \delta 7.18-7.53(\mathrm{~m}, 5 \mathrm{H}), 5.51(\mathrm{dq}, \mathrm{J}=15.2,6.5 \mathrm{~Hz}, 1 \mathrm{H}), 5.28(\mathrm{ddq}, \mathrm{J}=15.2,8.2,1.4 \mathrm{~Hz}, 1 \mathrm{H}), 5.09(\mathrm{~s}, 1 \mathrm{H})$, $4.89(\mathrm{~s}, 1 \mathrm{H}), 3.91(\mathrm{~d}, \mathrm{~J}=12.6 \mathrm{~Hz}, 1 \mathrm{H}), 3.70(\mathrm{~d}, \mathrm{~J}=12.6 \mathrm{~Hz}, 1 \mathrm{H}), 3.58(\mathrm{dt}, \mathrm{J}=8.2,6.2 \mathrm{~Hz}, 1 \mathrm{H}), 3.40(\mathrm{~s}, 2 \mathrm{H}), 1.69$ $(\mathrm{dd}, \mathrm{J}=6.5,1.4 \mathrm{~Hz}, 3 \mathrm{H}), 1.30-1.76(\mathrm{~m}, 6 \mathrm{H}), 0.92(\mathrm{t}, \mathrm{J}=7.4 \mathrm{~Hz}, 3 \mathrm{H}) \mathrm{ppm} ;{ }^{13} \mathrm{C} \mathrm{NMR}\left(75 \mathrm{MHz}, \mathrm{CDCl}_{3}\right): \delta 146.1$, 139.3, 132.2, 129.0, 128.3, 128.1, 126.0, 112.9, 79.9, 69.7, 40.0, 35.4, 27.7, 22.6, 17.6, 14.0 ppm; IR (KBr): 2932, 2859, 1651, 1495, 1453, 1073, 969, 902, 740, $699 \mathrm{~cm}^{-1}$; MS (EI, $\left.70 \mathrm{eV}\right): \mathrm{m} / \mathrm{z} 258\left(\mathrm{M}^{+}\right), 240,201,131,91,70$; HRMS $\mathrm{m} / \mathrm{z}$ calcd for $\mathrm{C}_{18} \mathrm{H}_{26} \mathrm{O}\left(\mathrm{M}^{+}\right): 258.1984$; found: 258.1988 .

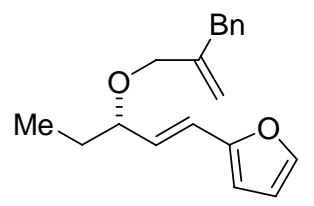

(S,E)-2-[3-(2-Benzylallyloxy)pent-1-enyl]furan (7d): General Procedure B was applied to (E)-3-(2-furyl)acrolein $(611 \mathrm{mg}, 5.0 \mathrm{mmol})$ and 2-benzylallyl acetate $(950 \mathrm{mg}, 5.0 \mathrm{mmol}){ }^{9}$ The crude product was purified by column chromatography on silica gel $(2 \%$ EtOAc in hexanes) to yield $765 \mathrm{mg}(54 \%)$ of the title compound as light yellow oil. $[\alpha]_{\mathrm{D}}=-89.7(\mathrm{c}$ 5.12, $\left.\mathrm{CHCl}_{3}\right) ;{ }^{1} \mathrm{H}$ NMR $\left(300 \mathrm{MHz}, \mathrm{CDCl}_{3}\right): \delta 7.27-7.41(\mathrm{~m}, 6 \mathrm{H}), 6.43(\mathrm{dd}, \mathrm{J}=3.2,1.9 \mathrm{~Hz}, 1 \mathrm{H}), 6.34(\mathrm{~d}, \mathrm{~J}=15.9 \mathrm{~Hz}$, $1 \mathrm{H}), 6.28(\mathrm{~d}, \mathrm{~J}=3.2 \mathrm{~Hz}, 1 \mathrm{H}), 6.07(\mathrm{dd}, \mathrm{J}=15.9,7.7 \mathrm{~Hz}, 1 \mathrm{H}), 5.19(\mathrm{~s}, 1 \mathrm{H}), 4.99(\mathrm{~s}, 1 \mathrm{H}), 4.04(\mathrm{~d}, \mathrm{~J}=12.5 \mathrm{~Hz}, 1 \mathrm{H})$, $3.84(\mathrm{~d}, \mathrm{~J}=12.5 \mathrm{~Hz}, 1 \mathrm{H}), 3.77(\mathrm{q}, \mathrm{J}=6.9 \mathrm{~Hz}, 1 \mathrm{H}), 3.48(\mathrm{~s}, 2 \mathrm{H}), 1.60-1.90(\mathrm{~m}, 5 \mathrm{H}), 1.04$ (t, J = 7.3 Hz, 3H) ppm; ${ }^{13} \mathrm{C}$ NMR $\left(75 \mathrm{MHz}, \mathrm{CDCl}_{3}\right): \delta 152.30,145.90,141.78,139.17,129.00,128.21,126.03,120.32,113.13,111.16$, 107.70, 80.87, 70.24, 40.00, 28.61, 9.84 ppm; IR (liquid film): 3027, 2964, 2931, 1651, 1494, 1453, 1030, 1013, 963, 736, $699 \mathrm{~cm}^{-1}$; MS (EI, $\left.70 \mathrm{eV}\right): \mathrm{m} / \mathrm{z} 282\left(\mathrm{M}^{+}\right), 253,225,200,191,151,148,135,131,123,115$; HRMS m/z calcd for $\mathrm{C}_{19} \mathrm{H}_{22} \mathrm{O}_{2}\left(\mathrm{M}^{+}\right)$282.1620; found: 282.1608. The allylic alcohol (S,E)-1-(furan-2-yl)pent-1-en-3-ol was purified by column chromatography on silica gel $\left(2 \%\right.$ EtOAc in hexanes); $[\alpha]_{\mathrm{D}}=-6.4\left(\mathrm{c} 1.74, \mathrm{CHCl}_{3}\right)$.

(8) 1-(2-(Bromomethyl)allyl)benzene was prepared by by reacting 2-benzylprop-2-en-1-ol with $\mathrm{PBr}_{3}$ in $\mathrm{CH}_{2} \mathrm{Cl}_{2}$. See Ref 2.

(9) 2-Benzylallyl acetate was prepared by reacting 2-benzylprop-2-en-1-ol with acetic anhydride. See Ref 2. 
Separating the enantiomers by chiral HPLC [Daicel Chiracel ${ }^{\mathrm{TM}}$ OD-H column, flow rate $0.5 \mathrm{~mL} / \mathrm{min}, 5 \% i$-PrOH, 95\% hexanes, $\mathrm{T}_{\mathrm{r}} 18.69(R)$ and $\left.20.52(S)\right]$ provided the enantiomer ratio: $3 R: 3 S=2.0: 98(96 \%$ ee).

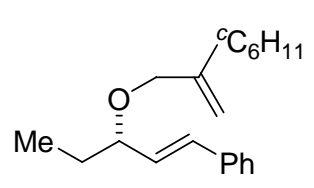

(S,E)-[3-(2-Cyclohexylallyloxy)pent-1-enyl]benzene (7e): General Procedure B was followed employing cinnamaldehyde $(2.0 \mathrm{mmol}, 264 \mathrm{mg})$ and 2-cyclohexylallyl acetate $(2.4$ mmol, $437 \mathrm{mg}) .{ }^{10} \quad$ The crude product was purified by flash chromatography on silica gel (2\% EtOAc in hexanes) to yield $341 \mathrm{mg}(60 \%)$ of the title compound as light yellow oil. $\quad[\alpha]$ ${ }_{\mathrm{D}}=-63.4\left(\mathrm{c} 4.51, \mathrm{CHCl}_{3}\right) ;{ }^{1} \mathrm{H}$ NMR $\left(300 \mathrm{MHz}, \mathrm{CDCl}_{3}\right): \delta 7.20-7.40(\mathrm{~m}, 5 \mathrm{H}), 6.58(\mathrm{~d}, \mathrm{~J}=15.9 \mathrm{~Hz}, 1 \mathrm{H}), 6.14(\mathrm{dd}, \mathrm{J}$ $=15.9,8.0 \mathrm{~Hz}, 1 \mathrm{H}), 5.09(\mathrm{~s}, 1 \mathrm{H}), 4.96(\mathrm{~s}, 1 \mathrm{H}), 4.14(\mathrm{~d}, \mathrm{~J}=12.8 \mathrm{~Hz}, 1 \mathrm{H}), 3.91(\mathrm{~d}, \mathrm{~J}=12.8 \mathrm{~Hz}, 1 \mathrm{H}), 3.84(\mathrm{q}, \mathrm{J}=6.9$ $\mathrm{Hz}, 1 \mathrm{H}), 1.98-2.08(\mathrm{~m}, 1 \mathrm{H}), 1.15-1.98(\mathrm{~m}, 12 \mathrm{H}), 1.03(\mathrm{t}, \mathrm{J}=7.4 \mathrm{~Hz}, 3 \mathrm{H}) \mathrm{ppm} ;{ }^{13} \mathrm{C}$ NMR $\left(75 \mathrm{MHz}, \mathrm{CDCl}_{3}\right): \delta$ $151.73,136.71,132.06,130.66,128.49,127.51,126.37,109.05,81.39,70.27,41.10,32.35,32.11,28.71,26.72$, 26.33, 9.90 ppm; IR (liquid film): 2926, 2852, 1449, 1093, 967, 891, 747, $692 \mathrm{~cm}^{-1}$; MS (EI, $70 \mathrm{eV}$ ): $\mathrm{m} / \mathrm{z}$ 284, 255, 193, 145, 129, 115, 91; HRMS $\mathrm{m} / \mathrm{z}$ calcd for $\mathrm{C}_{20} \mathrm{H}_{28} \mathrm{O}\left(\mathrm{M}^{+}\right)$: 284.2140; found: 284.2147. The allylic alcohol (S,E)-1-phenylpent-1-en-3-ol was purified by flash chromatography on silica gel (2\% EtOAc in hexanes). Separating the allylic alcohol enantiomers by chiral HPLC [Daicel Chiracel ${ }^{\mathrm{TM}}$ OD-H column, flow rate $1.0 \mathrm{~mL} / \mathrm{min}$, $0.5 \% \mathrm{i}-\mathrm{PrOH}, 99.5 \%$ hexanes, $\mathrm{T}_{\mathrm{r}} 13.4(R)$ and $\left.16.3(S)\right]$ provided the enantiomer ratio: $3 R: 3 S=3.5: 96.5(93 \%$ ee).

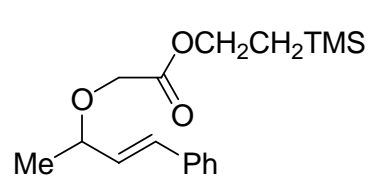

(E)-2-(Trimethylsilyl)ethyl-2-(4-phenylbut-3-en-2-yloxy)acetate (7f'): To a solution of (E)-2-(4-phenylbut-3-en-2-yloxy)acetic acid $(824 \quad \mathrm{mg}, \quad 4.0 \quad \mathrm{mmol}){ }^{11}$ and 2-(trimethylsilyl)ethanol (472 mg, $4.0 \mathrm{mmol})$ in $\mathrm{CH}_{2} \mathrm{Cl}_{2}(2 \mathrm{~mL})$ was added DCC (1.030 g, $5.0 \mathrm{mmol}$ ) and DMAP (43 $\mathrm{mg}, 0.4 \mathrm{mmol}$ ) and the reaction was stirred for $24 \mathrm{~h}$. The solvent was evaporated and the crude product mixture was purified by flash chromatography on silica gel $(10 \%$ EtOAc in hexanes) to yield $980 \mathrm{mg}(80 \%)$ of the title compound as a light yellow oil. ${ }^{1} \mathrm{H} \mathrm{NMR}(300 \mathrm{MHz}$, $\left.\mathrm{CDCl}_{3}\right): \delta 7.30-7.40(\mathrm{~m}, 5 \mathrm{H}), 6.53(\mathrm{~d}, \mathrm{~J}=16.0 \mathrm{~Hz}, 1 \mathrm{H}), 6.10(\mathrm{dd}, \mathrm{J}=16.0,8.0 \mathrm{~Hz}, 1 \mathrm{H}), 4.23(\mathrm{t}, \mathrm{J}=8.5 \mathrm{~Hz}, 2 \mathrm{H})$, $4.13(\mathrm{dq}, \mathrm{J}=8.0,6.3 \mathrm{~Hz}, 1 \mathrm{H}), 4.10(\mathrm{~d}, \mathrm{~J}=16.5 \mathrm{~Hz}, 1 \mathrm{H}), 4.08(\mathrm{~d}, \mathrm{~J}=16.5 \mathrm{~Hz}, 1 \mathrm{H}), 1.42$ (d, J = 6.3 Hz, 3H), 0.98 (t,

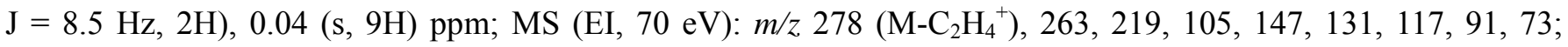
HRMS m/z calcd for $\mathrm{C}_{15} \mathrm{H}_{22} \mathrm{O}_{3} \mathrm{Si}\left(\mathrm{M}^{+}-\mathrm{C}_{2} \mathrm{H}_{4}\right): 278.1338$; found: 278.1330 .

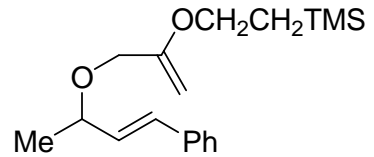

(E)-Trimethyl\{2-[3-(4-phenylbut-3-en-2-yloxy)prop-1-en-2-yloxy]ethyl\}silane (7f): To a $-40{ }^{\circ} \mathrm{C}$ solution of (E)-2-(trimethylsilyl)ethyl 2-(4-phenylbut-3-en-2-yloxy)acetate (7f', $612 \mathrm{mg}, 2.0 \mathrm{mmol}$ ) in $10 \mathrm{~mL}$ THF-pyridine (4:1) was added Tebbe's reagent (2. 5 equiv, $5.0 \mathrm{mmol}, 10 \mathrm{~mL}$ of $0.5 \mathrm{M}$ toluenesolution). ${ }^{12}$ Once addition was complete, the reaction mixture was warmed to ambient temperature and stirred for $16 \mathrm{~h}$. The reaction mixture was cooled to $0{ }^{\circ} \mathrm{C}$ and quenched by dropwise addition of $0.5 \mathrm{M}$ aq. $\mathrm{NaOH}$. The resulting mixture was filtered through a celite pad, washing the filter cake with $\mathrm{Et}_{2} \mathrm{O}$. The solvents were evaporated and the crude oil was purified by column chromatography on basic alumina (10\% $\mathrm{Et}_{2} \mathrm{O}$ in hexanes) to give $460 \mathrm{mg}$ of the title product $(75 \%)$ as colorless oil. ${ }^{1} \mathrm{H} \mathrm{NMR}\left(300 \mathrm{MHz}, \mathrm{CDCl}_{3}\right): \delta$ 7.12-7.31 (m, 5H), $6.53(\mathrm{~d}, \mathrm{~J}=16.0 \mathrm{~Hz}, 1 \mathrm{H}), 6.17(\mathrm{dd}, \mathrm{J}=16.0,7.5 \mathrm{~Hz}, 1 \mathrm{H}), 4.53$ (d, J = 0.5 Hz, 1H), $4.19(\mathrm{~s}, 1 \mathrm{H})$, $4.22(\mathrm{~d}, \mathrm{~J}=12.8 \mathrm{~Hz}, 1 \mathrm{H}), 4.04(\mathrm{~d}, \mathrm{~J}=12.8 \mathrm{~Hz}, 1 \mathrm{H}), 4.10(\mathrm{dq}, \mathrm{J}=7.5,6.3 \mathrm{~Hz}, 1 \mathrm{H}), 3.83(\mathrm{t}, \mathrm{J}=7.8 \mathrm{~Hz}, 2 \mathrm{H}), 1.41(\mathrm{~d}$,

(10) 2-Cyclohexylallyl acetate was prepared by reacting 2-cyclohexylprop-2-en-1-ol with acetic anhydride. See Ref 2.

(11) Prepared by reacting (E)-4-phenylbut-3-en-2-ol (1.0 equiv) with $\mathrm{NaH}$ (5.0 equiv), and 2-chloroacetic acid (1.0 equiv) in THF at reflux.

(12) For the in situ preparation of Tebbe's reagent: Pine, S. H.; Kim, G.; Lee, V. Org. Syn. 1990, 69, 72. 
$\mathrm{J}=6.3 \mathrm{~Hz}, 3 \mathrm{H}), 1.02(\mathrm{t}, \mathrm{J}=7.8 \mathrm{~Hz}, 2 \mathrm{H}), 0.04(\mathrm{~s}, 9 \mathrm{H}) \mathrm{ppm} ;{ }^{3} \mathrm{C} \mathrm{NMR}\left(75 \mathrm{MHz}, \mathrm{CDCl}_{3}\right): \delta 159.7,136.6,131.5,131.2$, 128.4, 127.5, 126.3, 82.5, 76.0, 68.6, 64.7, 21.6, 17.1, -1.5 ppm; IR (liquid film): 2954, 1663, 1250, 1071, 837, 749,

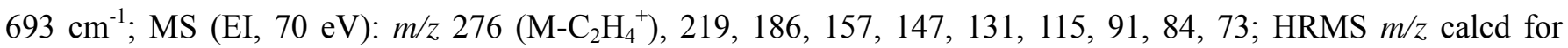
$\mathrm{C}_{16} \mathrm{H}_{24} \mathrm{O}_{2} \mathrm{Si}\left(\mathrm{M}^{+}-\mathrm{C}_{2} \mathrm{H}_{4}\right): 276.1546$; found: 276.1548 .

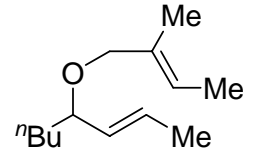

(2E)-4-[(E)-2-Methylbut-2-enyloxy]oct-2-ene (7g): General Procedure A was followed employing (E)-oct-2-en-4-ol (384 mg, $3.0 \mathrm{mmol})$ and (E)-1-bromo-2-methylbut-2-ene $(671 \mathrm{mg}$, $4.5 \mathrm{mmol})$ in THF. The crude product was purified by flash chromatography on silica gel $(2 \%$ EtOAc in hexanes) to yield $235 \mathrm{mg}(40 \%)$ of the title compound as a light yellow oil. ${ }^{1} \mathrm{H} \mathrm{NMR}(300 \mathrm{MHz}$, $\left.\mathrm{CDCl}_{3}\right): \delta 5.57(\mathrm{dq}, \mathrm{J}=15.3,6.4 \mathrm{~Hz}, 1 \mathrm{H}), 5.45(\mathrm{q}, \mathrm{J}=6.7 \mathrm{~Hz}, 1 \mathrm{H}), 5.29(\mathrm{ddq}, \mathrm{J}=15.3,8.2,1.5 \mathrm{~Hz}, 1 \mathrm{H}), 3.87(\mathrm{~d}, \mathrm{~J}$ $=11.2 \mathrm{~Hz}, 1 \mathrm{H}), 3.66(\mathrm{~d}, \mathrm{~J}=11.3 \mathrm{~Hz}, 1 \mathrm{H}), 3.55(\mathrm{q}, \mathrm{J}=7.0 \mathrm{~Hz}, 1 \mathrm{H}), 1.71(\mathrm{dd}, \mathrm{J}=6.4,1.5 \mathrm{~Hz}, 3 \mathrm{H}), 1.63(\mathrm{~s}, 3 \mathrm{H}), 1.61$ $(\mathrm{s}, 3 \mathrm{H}), 1.25-1.46(\mathrm{~m}, 6 \mathrm{H}), 0.89(\mathrm{t}, \mathrm{J}=6.8 \mathrm{~Hz}, 3 \mathrm{H}) \mathrm{ppm} ;{ }^{13} \mathrm{C} \mathrm{NMR}\left(75 \mathrm{MHz}, \mathrm{CDCl}_{3}\right): \delta 133.4,132.5,128.0,121.8$, 79.6, 73.8, 35.5, 27.7, 22.7, 17.6, 14.0, 13.8, 13.1 ppm; MS (EI, $70 \mathrm{eV}): \mathrm{m} / \mathrm{z} 196\left(\mathrm{M}^{+}\right), 181,139,110,81,71,69$; HRMS $\mathrm{m} / \mathrm{z}$ calcd for $\mathrm{C}_{13} \mathrm{H}_{24} \mathrm{O}$ 196.1827. found 196.1832 .

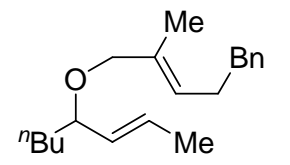

1-\{(3E)-5-[(E)-Oct-2-en-4-yloxy]-4-methylpent-3-enyl\}benzene (7h): General Procedure A was followed employing (E)-oct-2-en-4-ol (256 $\mathrm{mg}, 2.0 \mathrm{mmol})$ and a mixture of 1-[(E)-5-bromo-4-methylpent-3-enyl]benzene and 1-(3-bromo-4-methylpent-4-enyl)benzene $(\sim 4: 1,714 \mathrm{mg}, 3.0 \mathrm{mmol})^{13}$ in THF. The crude product was purified by flash chromatography on silica gel $(2 \%$ EtOAc in hexanes) to yield $372 \mathrm{mg}(65 \%)$ of the title compound as a light yellow oil. ${ }^{1} \mathrm{H} \mathrm{NMR}(300 \mathrm{MHz}$, $\left.\mathrm{CDCl}_{3}\right): \delta$ 7.18-7.53 (m, 5H), $5.55(\mathrm{dq}, \mathrm{J}=15.3,6.4 \mathrm{~Hz}, 1 \mathrm{H}), 5.44(\mathrm{t}, \mathrm{J}=6.8 \mathrm{~Hz}, 1 \mathrm{H}), 5.30$ (ddq, $\mathrm{J}=15.3,8.2,1.5$ $\mathrm{Hz}, 1 \mathrm{H}), 3.89$ (d, J = 11.5 Hz, 1H), 3.69 (d, J = 11.5 Hz, 1H), 3.55 (q, J = 7.0 Hz, 1H), 2.69 (t, J = 7.8 Hz, 2H), 2.39 $(\mathrm{q}, \mathrm{J}=7.4 \mathrm{~Hz}, 2 \mathrm{H}), 1.73(\mathrm{dd}, \mathrm{J}=6.2,1.0 \mathrm{~Hz}, 3 \mathrm{H}), 1.63(\mathrm{~s}, 3 \mathrm{H}), 1.24-1.50(\mathrm{~m}, 4 \mathrm{H}), 0.91(\mathrm{t}, \mathrm{J}=7.4 \mathrm{~Hz}, 3 \mathrm{H}) \mathrm{ppm} ;{ }^{13} \mathrm{C}$ NMR (75 MHz, $\left.\mathrm{CDCl}_{3}\right): \delta 142.1,133.3,132.4,128.4,128.2,128.1,126.6,125.7,79.4,73.6,35.7,35.4,31.6,29.6$, 27.7, 22.7, 17.7, $14.1 \mathrm{ppm}$; MS (EI, $70 \mathrm{eV}): \mathrm{m} / \mathrm{z} 286\left(\mathrm{M}^{+}\right), 229,195,181,175,160,143,127$; HRMS $\mathrm{m} / \mathrm{z}$ calcd for $\mathrm{C}_{16} \mathrm{H}_{21} \mathrm{O}\left(\mathrm{M}^{+}-\mathrm{C}_{4} \mathrm{H}_{9}\right) 229.1592$; found: 229.1594 .

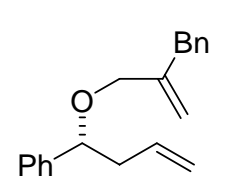

1-(2-\{[(R)-1-Phenylbut-3-enyloxy]methyl\}allyl)benzene (14): General Procedure A was followed employing (R)-1-phenylbut-3-en-1-ol $(91 \% \text { ee, } 296 \mathrm{mg}, 2.0 \mathrm{mmol})^{14}$ and [2-(bromomethyl)allyl]benzene $(630 \mathrm{mg}, 3.0 \mathrm{mmol})$ in THF. The crude product was purified by flash chromatography on silica gel (2\% EtOAc in hexanes) to yield $450 \mathrm{mg}(81 \%)$ of the title compound as light yellow oil. $+10.8\left(\mathrm{c} 1.07, \mathrm{CHCl}_{3}\right) ;{ }^{1} \mathrm{H}$ NMR $\left(300 \mathrm{MHz}, \mathrm{CDCl}_{3}\right)$ : $\delta$ 7.27-7.46 (m, 10H), 5.88-6.04 $(\mathrm{m}, 1 \mathrm{H}), 5.07-5.22(\mathrm{~m}, 3 \mathrm{H}), 5.00(\mathrm{~s}, 1 \mathrm{H}), 4.39(\mathrm{dd}, \mathrm{J}=7.4,5.8 \mathrm{~Hz}, 1 \mathrm{H}), 3.94(\mathrm{~d}, \mathrm{~J}=12.4 \mathrm{~Hz}, 1 \mathrm{H}), 3.76(\mathrm{~d}, \mathrm{~J}=12.4$ $\mathrm{Hz}, 1 \mathrm{H}), 3.54(\mathrm{~d}, \mathrm{~J}=15.0 \mathrm{~Hz}, 1 \mathrm{H}), 3.47(\mathrm{~d}, \mathrm{~J}=15.0 \mathrm{~Hz}, 1 \mathrm{H}), 2.62-2.82(\mathrm{~m}, 1 \mathrm{H}), 2.48-2.60(\mathrm{~m}, 1 \mathrm{H}) \mathrm{ppm} ;{ }^{13} \mathrm{C}$ NMR (75 MHz, $\mathrm{CDCl}_{3}$ ): $\delta$; IR (liquid film): 3027, 2908, 2856, 1642, 1601, 1494, 1453, 1087, 913, $699 \mathrm{~cm}^{-1}$; MS (EI, 70 $\mathrm{eV}): \mathrm{m} / \mathrm{z} 278\left(\mathrm{M}^{+}\right), 237,147,131,129,115,107$; HRMS m/z calcd for $\mathrm{C}_{20} \mathrm{H}_{22} \mathrm{O}\left(\mathrm{M}^{+}\right): 278.1671$; found 278.1675 .

(13) The mixture of 1-[(E)-5-bromo-4-methylpent-3-enyl]benzene and 1-(3-bromo-4-methylpent-4-enyl)benzene was prepared by reacting 2-methyl-5-phenylpent-1-en-3-ol with $\mathrm{PBr}_{3}$ in $\mathrm{CH}_{2} \mathrm{Cl}_{2}$. See Ref 2.

(14) The enantioenriched homoallylic alcohol was prepared according to the published procedure, see: Brown, H. C.; J. Am. Chem. Soc. 1990, 112, 2389. 


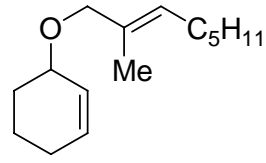

(E)-3-(2-Methyloct-2-enyloxy)cyclohex-1-ene (16a): General Procedure A was followed employing $0.49 \mathrm{~g}$ of cyclohex-2-enol $(5.0 \mathrm{mmol}), 240 \mathrm{mg} \mathrm{NaH}(10.0 \mathrm{mmol})$ and $1.03 \mathrm{~g}$ of a 4:1 mixture of (E)-1-bromo-2-methyloct-2-ene and 3-bromo-2-methyloct-1-ene $(5.0 \mathrm{mmol})^{15}$ in THF. Purifying the crude product mixture by flash chromatography (2\% EtOAc in hexanes) gave $440 \mathrm{mg}(40 \%)$ of the title compound as colorless oil. ${ }^{1} \mathrm{H}$ NMR $\left(300 \mathrm{MHz}, \mathrm{CDCl}_{3}\right): \delta 5.83(\mathrm{~d}, \mathrm{~J}=10.1 \mathrm{~Hz}, 1 \mathrm{H}), 5.75(\mathrm{~d}, \mathrm{~J}=10.1$ $\mathrm{Hz}, 1 \mathrm{H}), 5.41(\mathrm{t}, \mathrm{J}=6.8 \mathrm{~Hz}, 1 \mathrm{H}), 3.93(\mathrm{~d}, \mathrm{~J}=11.4 \mathrm{~Hz}, 1 \mathrm{H}), 3.87$ (d, J = 11.4 Hz, 1H), 3.83-3.87 (m, 1H), 1.90-2.03 $(\mathrm{m}, 4 \mathrm{H}), 1.29-1.79(\mathrm{~m}, 10 \mathrm{H}), 1.65(\mathrm{~s}, 3 \mathrm{H}), 0.88(\mathrm{t}, \mathrm{J}=7.4 \mathrm{~Hz}, 3 \mathrm{H}) \mathrm{ppm} ;{ }^{13} \mathrm{C} \mathrm{NMR}\left(300 \mathrm{MHz}, \mathrm{CDCl}_{3}\right): \delta 132.44$, 130.47, 128.21, 128.06, 74.32, 71.27, 31.53, 29.13, 28.33, 27.63, 25.20, 22.53, 19.27, 14.02, 13.92 ppm; MS (EI, $70 \mathrm{ev}): \mathrm{m} / \mathrm{z} 222\left(\mathrm{M}^{+}\right), 207,179,127,107,97,81,69$; HRMS $\mathrm{m} / \mathrm{z}$ calcd for $\mathrm{C}_{15} \mathrm{H}_{26} \mathrm{O}\left(\mathrm{M}^{+}\right): 222.1984$; found: 222.1978 .

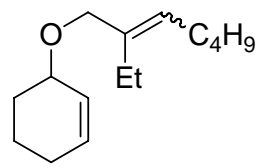

3-(2-Ethylhept-2-enyloxy)cyclohex-1-ene (16b): General Procedure A was followed employing $0.20 \mathrm{~g}$ of cyclohex-2-enol $(2.0 \mathrm{mmol}), 144 \mathrm{mg} \mathrm{NaH}(6.0 \mathrm{mmol})$, and $715 \mathrm{mg}$ of a mixture of $(E / Z)$-3-(bromomethyl)oct-3-ene and 4-bromo-3-methyleneoctane $(3.0 \mathrm{mmol})^{16}$ in

THF. Purifying the crude product mixture by flash chromatography (2\% EtOAc/hexanes) gave $176 \mathrm{mg}(40 \%)$ of the title compound as a mixture of $\mathrm{E}$ and $\mathrm{Z}$ isomers $(\mathrm{E}: \mathrm{Z}=1: 3) .{ }^{1} \mathrm{H} \mathrm{NMR}\left(300 \mathrm{MHz}, \mathrm{CDCl}_{3}\right): \delta 5.83(\mathrm{dt}, \mathrm{J}=10.2$, $3.5 \mathrm{~Hz}, 1 \mathrm{H}), 5.76(\mathrm{~d}, \mathrm{~J}=10.2 \mathrm{~Hz}, 1 \mathrm{H}), 5.38$ (t, J = 7.2 Hz, 1H), 3.97 (d, J = 11.6 Hz, 1H), 3.89 (d, J = 11.6 Hz, 1H), 1.20-2.10 (m, 14H), $0.90(\mathrm{t}, \mathrm{J}=7.4 \mathrm{~Hz}, 3 \mathrm{H}), 1.01(\mathrm{t}, \mathrm{J}=7.4 \mathrm{~Hz}, 3 \mathrm{H}) \mathrm{ppm} ; \mathrm{MS}(\mathrm{EI}, 70 \mathrm{ev}): \mathrm{m} / \mathrm{z} 222\left(\mathrm{M}^{+}\right), 124,95$, 85, 81, 69; HRMS m/z calcd for $\mathrm{C}_{15} \mathrm{H}_{26} \mathrm{O}\left(\mathrm{M}^{+}\right)$: 222.1984; found 222.1978.

General Procedure C for ICR of di(allyl)ethers: A solution of $\left[\operatorname{IrCl}\left(\mathrm{C}_{8} \mathrm{H}_{14}\right)_{2}\right]_{2}$ (0.01 equiv or indicated amount) and $\mathrm{PCy}_{3}\left(6\right.$ equiv relative to $\left.\left[\mathrm{IrCl}\left(\mathrm{C}_{8} \mathrm{H}_{14}\right)_{2}\right]_{2}\right)$ in anhydrous $\mathrm{CH}_{2} \mathrm{Cl}_{2}$ or 1,2-dichloroethane $(1.5 \mathrm{~mL})$ was added to a solution of $\mathrm{NaBPh}_{4}\left(2\right.$ equiv relative to $\left.\left[\mathrm{IrCl}\left(\mathrm{C}_{8} \mathrm{H}_{14}\right)_{2}\right]_{2}\right)$ in $\mathrm{CH}_{2} \mathrm{Cl}_{2}$ /acetone or 1,2-dichloroethane/acetone (25:1) (1.5 $\mathrm{mL}, 0.67 \mathrm{M}$ final concentration in substrate) and the resulting yellow solution stirred for $5 \mathrm{~min}$ at ambient temperature. The diallyl ether (1.0 equiv) was added and the reaction was heated at reflux for the indicated time. After cooling to ambient temperature, the solvent was evaporated, and the crude product mixture was purified as indicated.

Determination of Claisen Rearrangement Stereoselectivity: Stereoisomer ratios for Claisen adducts were determined by HPLC, GLC, and/or integration of the aldehyde $\mathrm{C}-\mathbf{H}$ resonance in the ${ }^{1} \mathrm{H}$ NMR spectrum. For substrates where accurate ratios could not be directly determined for the Claisen adducts, these products were reduced to the corresponding primary alcohols and the stereoisomer ratios determined by HPLC or GLC. Experimental procedures and characterization data for these primary alcohols is provided.

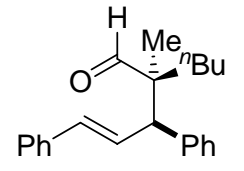

anti-2-[(E)-1,3-Diphenylallyl]-2-methylhexanal (8a): General Procedure $C$ was followed employing $0.477 \mathrm{~g}$ of (E)-3-(2-methylenehexyloxy)-1,3-diphenylprop-1-ene (7a, $6.21 \mathrm{mmol})$ in $\mathrm{CH}_{2} \mathrm{Cl}_{2}$ at $23{ }^{\circ} \mathrm{C}$. Purifying the crude product mixture by MPLC (0-10\% EtOAc in hexanes)

(15) The mixture of (E)-1-bromo-2-methyloct-2-ene and 3-bromo-2-methyloct-1-ene was prepared by reacting 2-methyloct-1-en-3-ol with $\mathrm{PBr}_{3}$ in $\mathrm{CH}_{2} \mathrm{Cl}_{2}$. See Ref 2.

(16) The mixture of (E/Z)-3-(bromomethyl)oct-3-ene and 4-bromo-3-methyleneoctan was prepared by reacting 3-methyleneoctan-4-ol with $\mathrm{PBr}_{3}$ in $\mathrm{CH}_{2} \mathrm{Cl}_{2}$. See Ref 2. 
afforded $0.430 \mathrm{~g}(90 \%)$ of the title compound as a colorless oil (anti:syn $=97: 3) .{ }^{1} \mathrm{H}$ NMR $\left(300 \mathrm{MHz}, \mathrm{CDCl}_{3}\right)$ : $\delta 0.87(\mathrm{~m}, 3 \mathrm{H}), 1.12(\mathrm{~s}, 3 \mathrm{H}), 1.25(\mathrm{~m}, 4 \mathrm{H}), 1.47-1.77(\mathrm{~m}, 2 \mathrm{H}), 3.68(\mathrm{~d}, \mathrm{~J}=8.7 \mathrm{~Hz}, 1 \mathrm{H}), 6.47(\mathrm{~d}, \mathrm{~J}=16 \mathrm{~Hz}, 1 \mathrm{H})$, $6.56(\mathrm{dd}, \mathrm{J}=16,8.7 \mathrm{~Hz}, 1 \mathrm{H}), 7.17-7.39(\mathrm{~m}, 10 \mathrm{H}), 9.61(\mathrm{~s}, 1 \mathrm{H}) ;{ }^{13} \mathrm{C} \mathrm{NMR}\left(75 \mathrm{MHz}, \mathrm{CDCl}_{3}\right): \delta 13.9,16.0,23.3$, 26.3, 34.6, 52.7, 55.7, 126.3, 126.8, 127.4, 127.8, 128.3, 128.5, 129.0, 133.0, 137.1, 140.2, 206.4; MS (EI, 70 eV): $\mathrm{m} / \mathrm{z} 306\left(\mathrm{M}^{+}\right), 250,193,178,165,115,91$; HRMS m/z calcd for $\mathrm{C}_{22} \mathrm{H}_{26} \mathrm{O}\left(\mathrm{M}^{+}\right): 306.1984$; found: 306.1986.

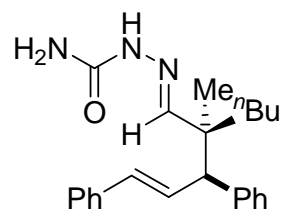

anti-(1E)-1-[(E)-2-Butyl-2-methyl-3,5-diphenylpent-4-enylidene]semicarbazide

$\left(8 \mathbf{a}^{\prime}\right)$ :

Semicarbazide hydrochloride $(1.11 \mathrm{~g}, 10 \mathrm{mmol})$ and dry sodium acetate $(1.23 \mathrm{~g}, 15 \mathrm{mmol})$ were ground together in a mortar. Ethanol $(10 \mathrm{~mL})$ was added and the resulting mixture was heated to boiling. The hot solution was filtered and then cooled to ambient temperature. anti-2-[(E)-1,3-diphenylallyl]-2-methylhexanal $(8 \mathbf{a}, 153 \mathrm{mg}, 0.5 \mathrm{mmol})$ was added and the mixture was heated at $40{ }^{\circ} \mathrm{C}$ for $2.5 \mathrm{~h}$. Water $(20 \mathrm{~mL})$ was added and the resulting mixture was extracted with ethyl ether $(3 \times 20 \mathrm{~mL})$. Evaporating the solvent gave $181 \mathrm{mg}$ of the desired product $(100 \%)$ that was recrystallized from ethanol to provide crystals suitable for X-ray diffraction analysis. ${ }^{1} \mathrm{H}$ NMR $\left(300 \mathrm{MHz}, \mathrm{DMSO}-\mathrm{d}^{6}\right): \delta 9.77(\mathrm{~s}, 1 \mathrm{H}), 6.70(\mathrm{dd}, \mathrm{J}=15.6$, $10.1 \mathrm{~Hz}, 1 \mathrm{H}), 6.43$ (d, J = 15.6 Hz, 1H), 6.02 (s, 2H), 3.51 (d, J = 9.9 Hz, 1H), $3.33(\mathrm{~s}, 1 \mathrm{H}), 1.10-1.45$ (m, 7H), 1.05 $(\mathrm{s}, 3 \mathrm{H}), 0.79(\mathrm{t}, \mathrm{J}=7.4 \mathrm{~Hz}, 3 \mathrm{H}) \mathrm{ppm} ;{ }^{13} \mathrm{C}$ NMR $\left(75 \mathrm{MHz}, \mathrm{DMSO}-\mathrm{d}^{6}\right): \delta 156.71,148.56,141.41,137.04,131.47$, $129.86,129.20,128.50,127.80,127.18,126.15,126.10,57.69,43.66,37.06,25.91,22.88,19.21,13.97$ ppm; MS (EI, $70 \mathrm{eV}): \mathrm{m} / \mathrm{z} 363\left(\mathrm{M}^{+}\right), 246,233,193,178,165,127,115,91$; HRMS $\mathrm{m} / \mathrm{z}$ calcd for $\mathrm{C}_{23} \mathrm{H}_{29} \mathrm{ON}_{3}\left(\mathrm{M}^{+}\right): 363.2311$; found: 363.2237. See Appendix for X-ray diffraction data.

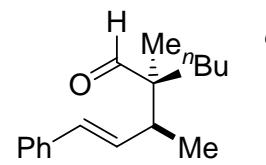

anti-2-Methyl-2-[(E)-4-phenylbut-3-en-2-yl]hexanal (8b): General Procedure $\mathbf{C}\left(\mathrm{CH}_{2} \mathrm{Cl}_{2}\right.$ at 40 ${ }^{\circ} \mathrm{C}$ ) was followed employing $0.491 \mathrm{~g}$ of 1-[(E)-1-(2-methylenehexyloxy)but-2-enyl]benzene (7b). Purifying the crude product mixture by MPLC (0-10\% gradient EtOAc in hexanes) afforded 454 $\mathrm{mg}(93 \%)$ of the title compound as a colorless oil (anti:syn = 93:7). Separating the steoisomers by GLC [Varian CP Wax $52 \mathrm{CB}$ column $\left(30 \mathrm{~m} \times 0.25 \mathrm{~mm}\right.$ ), flow rate $0.6 \mathrm{~mL} / \mathrm{min}$, method: $80{ }^{\circ} \mathrm{C}$ for $2.00 \mathrm{~min}$, ramp @ $5.00{ }^{\circ} \mathrm{C} / \mathrm{min}$ to $250{ }^{\circ} \mathrm{C}$, hold for $20.00 \mathrm{~min}, \mathrm{Tr}=55.4$ (anti), 55.8 (syn) $\mathrm{min}$ ] provided the diastereomer ratio: 93:7. ${ }^{1} \mathrm{H}$ NMR (300 MHz, $\left.\mathrm{CDCl}_{3}\right): \delta 0.87(\mathrm{~m}, 3 \mathrm{H}), 1.01(\mathrm{~d}, \mathrm{~J}=5.8 \mathrm{~Hz}, 3 \mathrm{H}), 1.03(\mathrm{~s}, 3 \mathrm{H}), 1.03-1.25(\mathrm{~m}, 4 \mathrm{H}), 1.43-1.63(\mathrm{~m}, 2 \mathrm{H})$, $2.61(\mathrm{~m}, 1 \mathrm{H}), 6.08(\mathrm{dd}, \mathrm{J}=16,9.0 \mathrm{~Hz}, 1 \mathrm{H}), 6.43(\mathrm{~d}, \mathrm{~J}=16 \mathrm{~Hz}, 1 \mathrm{H}), 7.21-7.40(\mathrm{~m}, 5 \mathrm{H}), 9.50(\mathrm{~s}, 1 \mathrm{H}) ;{ }^{13} \mathrm{C}$ NMR $(75$ $\left.\mathrm{MHz}, \mathrm{CDCl}_{3}\right): \delta 13.6,13.9,16.0,23.3,26.1,34.9,41.8,52.2,126.1,127.2,128.5,130.4,131.3,137.2,206.8$; MS (EI, $70 \mathrm{eV}): \mathrm{m} / \mathrm{z} 244\left(\mathrm{M}^{+}\right), 188,131,115,104,91,77,70,65,55 ;$ HRMS $\mathrm{m} / \mathrm{z}$ calcd for $\mathrm{C}_{17} \mathrm{H}_{24} \mathrm{O}\left(\mathrm{M}^{+}\right): 244.1827$; found: 244.1827 .

anti-(E)-2-Benzyl-2,3-dimethylnon-4-enal (8c): General Procedure $\mathbf{C}$ was followed employing
$1-(2-\{[(E)-$ oct-2-en-4-yloxy)methyl $]$ allyl $\}$ benzene $(7 c, 258 \mathrm{mg}, 1.0 \mathrm{mmol})$ and $2 \mathrm{~mol} \%$ of the $75^{\circ} \mathrm{C}$ using 1,2-dichloroethane as solvent. Purifying the crude product mixture by flash chromatography on silica gel $\left(2.5 \% \mathrm{Et}_{2} \mathrm{O}\right.$ in hexanes) yielded $194 \mathrm{mg}(75 \%)$ of the title compound as a colorless oil (anti:syn: $\left.Z_{\text {syn }}=95: 3: 2\right)$. Separating the stereoisomers by GLC [Varian CP Wax 52CB column $(30 \mathrm{~m} \times 0.25 \mathrm{~mm})$, flow rate $0.6 \mathrm{~mL} / \mathrm{min}$, method: $80{ }^{\circ} \mathrm{C}$ for $2.00 \mathrm{~min}$, ramp @ $5.00{ }^{\circ} \mathrm{C} / \mathrm{min}$ to $250{ }^{\circ} \mathrm{C}$, hold for $20.00 \mathrm{~min}$, $\mathrm{Tr}=50.4,50.6,53.0$ and $53.4 \mathrm{~min}$ ] provided the diastereomer ratio: $Z_{\text {syn }}$ syn:anti $=2.6: 2.9: 94.5 .{ }^{1} \mathrm{H}$ NMR $\left(300 \mathrm{MHz}, \mathrm{CDCl}_{3}\right): \delta 9.61(\mathrm{~s}, 1 \mathrm{H})$, 7.04-7.28 (m, 5H), $5.56(\mathrm{dt}, \mathrm{J}=15.2,6.7 \mathrm{~Hz}, 1 \mathrm{H}), 5.38(\mathrm{ddt}, \mathrm{J}=15.2,8.8,1.2 \mathrm{~Hz}, 1 \mathrm{H}), 3.01(\mathrm{~d}, \mathrm{~J}=13.8 \mathrm{~Hz}, 1 \mathrm{H})$, $2.70(\mathrm{~d}, \mathrm{~J}=13.8 \mathrm{~Hz}, 1 \mathrm{H}), 2.49(\mathrm{dq}, \mathrm{J}=8.6,7.0 \mathrm{~Hz}, 1 \mathrm{H}), 2.06-2.13(\mathrm{~m}, 2 \mathrm{H}), 1.36-1.42(\mathrm{~m}, 4 \mathrm{H}), 0.99(\mathrm{~d}, \mathrm{~J}=6.9 \mathrm{~Hz}$, 
$3 \mathrm{H}), 0.95(\mathrm{t}, \mathrm{J}=7.4 \mathrm{~Hz}, 3 \mathrm{H}), 0.93(\mathrm{~s}, 3 \mathrm{H}) \mathrm{ppm} ;{ }^{13} \mathrm{C} \mathrm{NMR}\left(75 \mathrm{MHz}, \mathrm{CDCl}_{3}\right): \delta 207.0,137.2,133.2,130.2,129.9$, 128.1, 126.4, 53.3, 41.8, 41.4, 32.3, 31.6, 22.2, 16.4, 13.9, 13.7 ppm; MS (EI, $70 \mathrm{eV}): \mathrm{m} / \mathrm{z} 258\left(\mathrm{M}^{+}\right), 240,201,131$, 91, 70; HRMS m/z calcd for $\mathrm{C}_{18} \mathrm{H}_{26} \mathrm{O}\left(\mathrm{M}^{+}\right): 258.1984$; found: 258.1988 .

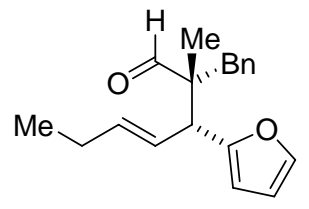

(2R,3R,E)-2-Benzyl-3-(furan-2-yl)-2-methylhept-4-enal (8d): General Procedure C was followed employing di(allyl) ether $7 \mathbf{d}(141 \mathrm{mg}, 0.5 \mathrm{mmol})$ and $4 \mathrm{~mol} \%$ catalyst $(9.0 \mathrm{mg}$ $\left[\mathrm{IrCl}\left(\mathrm{C}_{8} \mathrm{H}_{14}\right)_{2}\right]_{2}, 16.8 \mathrm{mg} \mathrm{PCy}$, and $6.8 \mathrm{mg} \mathrm{NaBPh}$ ) using 1,2-dichloroethane as solvent at 35 ${ }^{\circ} \mathrm{C}$ and an initial reaction time of $12 \mathrm{~h}$. After addition of $15.6 \mathrm{mg} \mathrm{PPh}_{3}$, the reaction was heated at $80{ }^{\circ} \mathrm{C}$ for $16 \mathrm{~h} ;(2 R, 3 R):(2 S, 3 R)=96: 4$. Purifying the crude product mixture by flash chromatography on silica gel $\left(2.5 \% \mathrm{Et}_{2} \mathrm{O}\right.$ in hexanes) yielded $107 \mathrm{mg}(76 \%)$ of the title compound as a light yellow oil. $[\alpha]_{\mathrm{D}}=+25.0(\mathrm{c}$ 2.71, $\left.\mathrm{CHCl}_{3}\right) ;{ }^{1} \mathrm{H}$ NMR $\left(300 \mathrm{MHz}, \mathrm{CDCl}_{3}\right): \delta 9.75(\mathrm{~s}, 1 \mathrm{H}), 7.05-7.40(\mathrm{~m}, 6 \mathrm{H}), 6.32(\mathrm{dd}, \mathrm{J}=3.1,1.9 \mathrm{~Hz}, 1 \mathrm{H}), 6.10$ $(\mathrm{d}, \mathrm{J}=3.1 \mathrm{~Hz}, 1 \mathrm{H}), 5.61-5.76(\mathrm{~m}, 2 \mathrm{H}), 3.72(\mathrm{~d}, \mathrm{~J}=8.2 \mathrm{~Hz}, 1 \mathrm{H}), 3.07(\mathrm{~d}, \mathrm{~J}=13.7 \mathrm{~Hz}, 1 \mathrm{H}), 2.75(\mathrm{~d}, \mathrm{~J}=13.7 \mathrm{~Hz}, 1 \mathrm{H})$, $2.15(\mathrm{qd}, \mathrm{J}=7.4,5.0 \mathrm{~Hz}, 1 \mathrm{H}), 1.06(\mathrm{t}, \mathrm{J}=7.4 \mathrm{~Hz}, 3 \mathrm{H}), 0.99(\mathrm{~s}, 3 \mathrm{H}) \mathrm{ppm} ;{ }^{13} \mathrm{C}$ NMR $\left(75 \mathrm{MHz}, \mathrm{CDCl}_{3}\right): \delta 205.87$, $154.44,141.49,137.40,136.72,130.38,128.11,126.48,123.71,110.11,107.33,53.28,48.55,41.05,25.64,15.98$, 13.64 ppm; IR (liquid film): 2965, 2932, 1723, 1497, 1454, 1148, 1013, 973, 733, $701 \mathrm{~cm}^{-1}$; MS (EI, $70 \mathrm{eV}$ ): $\mathrm{m} / \mathrm{z}$ $282\left(\mathrm{M}^{+}\right), 253,225,200,191,151,148,135,131,123,115$; HRMS $m / z$ calcd for $\mathrm{C}_{19} \mathrm{H}_{22} \mathrm{O}_{2}\left(\mathrm{M}^{+}\right): 282.1620$; found: 282.1608 .

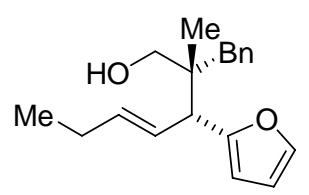

(2R,3R,E)-2-Benzyl-3-(furan-2-yl)-2-methylhept-4-en-1-ol (8d'): A 1 M hexanes solution of ${ }^{i} \mathrm{Bu}_{2} \mathrm{AlH}(0.5 \mathrm{~mL}, 0.5 \mathrm{mmol})$ was added to a $-78{ }^{\circ} \mathrm{C}$ solution of aldehyde $8 \mathbf{d}(70 \mathrm{mg}, 0.25$ $\mathrm{mmol}$ ) in $\mathrm{CH}_{2} \mathrm{Cl}_{2}$. After stirring $30 \mathrm{~min}$ at $-78{ }^{\circ} \mathrm{C}$, a saturated aqueous sodium tartrate solution $(2 \mathrm{~mL})$ was added and the mixture was warmed to ambient temperature and stirred until two homogeneous phases formed $(\sim 1 \mathrm{~h})$. The mixture was extracted with $\mathrm{Et}_{2} \mathrm{O}(3 \times)$ and the combined organic extracts were dried $\left(\mathrm{MgSO}_{4}\right)$, concentrated, and the resulting crude oil was purified by flash chromatography on silica gel (16\% EtOAc in hexanes) to yield $56 \mathrm{mg}(80 \%)$ of the title compound as a light yellow oil. Separating the enantiomers by chiral HPLC [Daicel Chiracel ${ }^{\mathrm{TM}}$ OD-H column, flow rate $0.5 \mathrm{~mL} / \mathrm{min}, 5.0 \%$ $i-\mathrm{PrOH}, 95.0 \%$ hexanes, $\mathrm{T}_{\mathrm{r}} 10.06(2 R, 3 R)$ and $\left.11.12(2 S, 3 S)\right]$ provided the enantiomer ratio: $(2 R, 3 R)-8 \mathbf{d}^{\prime}:(2 S$, $3 S)-8 d^{\prime}=97.6: 2.4(95.2 \%$ ee $) . \quad[\alpha]_{\mathrm{D}}=+18.1\left(\mathrm{c} 5.37, \mathrm{CHCl}_{3}\right) ;{ }^{1} \mathrm{H}$ NMR $\left(300 \mathrm{MHz}, \mathrm{CDCl}_{3}\right): \delta 7.37(\mathrm{dd}, \mathrm{J}=1.7,0.6$ $\mathrm{Hz}, 1 \mathrm{H}), 7.20-7.35(\mathrm{~m}, 5 \mathrm{H}), 6.36(\mathrm{dd}, \mathrm{J}=3.1,1.7 \mathrm{~Hz}, 1 \mathrm{H}), 6.12(\mathrm{~d}, \mathrm{~J}=3.1 \mathrm{~Hz}, 1 \mathrm{H}), 5.80(\mathrm{dd}, \mathrm{J}=15.2,9.5 \mathrm{~Hz}, 1 \mathrm{H})$, $5.66(\mathrm{dt}, \mathrm{J}=15.2,6.0 \mathrm{~Hz}, 1 \mathrm{H}), 3.61(\mathrm{~d}, \mathrm{~J}=9.5 \mathrm{~Hz}, 1 \mathrm{H}), 3.19(\mathrm{~d}, \mathrm{~J}=11.5 \mathrm{~Hz}, 1 \mathrm{H}), 3.06(\mathrm{~d}, \mathrm{~J}=11.5 \mathrm{~Hz}, 1 \mathrm{H}), 2.87$ (d, $\mathrm{J}=13.2 \mathrm{~Hz}, 1 \mathrm{H}), 2.63(\mathrm{~d}, \mathrm{~J}=13.2 \mathrm{~Hz}, 1 \mathrm{H}), 2.14(\mathrm{qd}, \mathrm{J}=7.4,6.0 \mathrm{~Hz}, 2 \mathrm{H}), 1.06(\mathrm{t}, \mathrm{J}=7.4 \mathrm{~Hz}, 3 \mathrm{H}), 0.73(\mathrm{~s}, 3 \mathrm{H})$ ppm; ${ }^{13} \mathrm{C}$ NMR $\left(75 \mathrm{MHz}, \mathrm{CDCl}_{3}\right): \delta 156.34,140.78,138.58,135.81,130.80,127.81,125.89,125.42,110.22$, 106.52, 66.05, 48.63, 42.86, 40.73, 25.70, 17.70, 13.76 ppm; IR (liquid film): 3590, 3461, 2964, 2247, 1496, 1454, 1147, 1032, 973, 910, 731, $703 \mathrm{~cm}^{-1}$; MS (EI, $\left.70 \mathrm{eV}\right): \mathrm{m} / \mathrm{z} 284\left(\mathrm{M}^{+}\right), 193,148,135,121,117,107,91$, 79; HRMS $\mathrm{m} / \mathrm{z}$ calcd for $\mathrm{C}_{19} \mathrm{H}_{24} \mathrm{O}_{2}\left(\mathrm{M}^{+}\right): 284.1776$; found: 284.1787 .

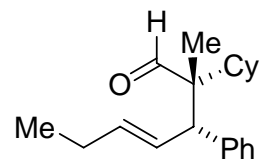

(2R,3S,E)-2-Cyclohexyl-2-methyl-3-phenylhept-4-enal (8e): General Procedure C was followed employing di(allyl) ether 7 e $(142 \mathrm{mg}, 0.5 \mathrm{mmol})$ and $4 \mathrm{~mol} \%$ catalyst $3(9.0 \mathrm{mg}$ $\left[\operatorname{IrCl}\left(\mathrm{C}_{8} \mathrm{H}_{14}\right)_{2}\right]_{2}, 16.8 \mathrm{mg} \mathrm{PCy}_{3}$, and $\left.6.8 \mathrm{mg} \mathrm{NaBPh}_{4}\right)$ in 1,2-dichloroethane solvent at $50{ }^{\circ} \mathrm{C}$ and an initial reaction time of $12 \mathrm{~h}$. After addition of $15.6 \mathrm{mg} \mathrm{PPh}_{3}$, the reaction was heated at $80{ }^{\circ} \mathrm{C}$ for $16 \mathrm{~h}$; $(2 \mathrm{R}, 3 \mathrm{~S}):(2 \mathrm{~s}, 3 \mathrm{~S})=87: 13$. Purifying the crude product mixture by flash chromatography on silica gel $\left(2.5 \% \mathrm{Et}_{2} \mathrm{O}\right.$ in hexanes) yielded $100 \mathrm{mg}(70 \%)$ of the title compound as a light yellow oil. Stereoisomer ratios were determined 
for corresponding primary alcohol (see below). $[\alpha]_{\mathrm{D}}=+17\left(\mathrm{c} 0.78, \mathrm{CHCl}_{3}\right) ;{ }^{1} \mathrm{H} \mathrm{NMR}\left(300 \mathrm{MHz}, \mathrm{CDCl}_{3}\right): \delta 9.66$ (s, 1H), 7.20-7.40 (m, 5H), $5.66(\mathrm{dd}, \mathrm{J}=15.1,9.5 \mathrm{~Hz}, 1 \mathrm{H}), 5.56(\mathrm{dt}, \mathrm{J}=15.1,5.8 \mathrm{~Hz}, 1 \mathrm{H}), 3.70(\mathrm{~d}, \mathrm{~J}=9.5 \mathrm{~Hz}, 1 \mathrm{H})$, 1.98-2.13 (m, 2H), 1.12-1.80 (m, $13 \mathrm{H}), 0.99(\mathrm{~s}, 3 \mathrm{H}), 0.98(\mathrm{t}, \mathrm{J}=7.4 \mathrm{~Hz}, 3 \mathrm{H}) \mathrm{ppm} ;{ }^{13} \mathrm{C}$ NMR $\left(75 \mathrm{MHz}, \mathrm{CDCl}_{3}\right): \delta$ 208.33, 141.08, 135.33, 128.99, 128.21, 127.22, 126.49, 54.40, 52.91, 40.99, 28.36, 27.19, 26.80, 26.64, 26.49, 25.63, 13.71, $13.51 \mathrm{ppm}$; IR (liquid film): 2927, 2853, 1722, 1451, 969, 746, $702 \mathrm{~cm}^{-1}$; MS (EI, $70 \mathrm{eV}$ ): $\mathrm{m} / \mathrm{z} 284$ $\left(\mathrm{M}^{+}\right), 255,193,145,129,115,91$; HRMS m/z calcd for $\mathrm{C}_{20} \mathrm{H}_{28} \mathrm{O}\left(\mathrm{M}^{+}\right)$: 284.2140; found: 284.2145.

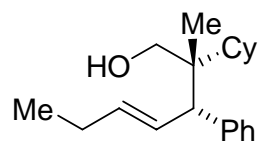

(2R,3S,E)-2-Cyclohexyl-2-methyl-3-phenylhept-4-en-1-ol (8e'): A 1 M hexanes solution of ${ }^{i} \mathrm{Bu}_{2} \mathrm{AlH}(0.5 \mathrm{~mL}, 0.5 \mathrm{mmol})$ was added to a $-78{ }^{\circ} \mathrm{C}$ solution of aldehyde $8 \mathrm{e}(70 \mathrm{mg}, 0.25 \mathrm{mmol})$ in $\mathrm{CH}_{2} \mathrm{Cl}_{2}$. After stirring $30 \mathrm{~min}$ at $-78{ }^{\circ} \mathrm{C}$, a saturated aqueous sodium tartrate solution $(2 \mathrm{~mL})$ was added and the mixture was warmed to ambient temperature and stirred until two homogeneous phases formed $(\sim 1 \mathrm{~h})$. The mixture was extracted with $\mathrm{Et}_{2} \mathrm{O}(3 \times)$ and the combined organic extracts were dried $\left(\mathrm{MgSO}_{4}\right)$, concentrated, and the resulting crude oil was purified by flash chromatography on silica gel (16\% EtOAc in hexanes) to yield $59 \mathrm{mg}(85 \%)$ of the title compound as a light yellow oil. Separating the stereoisomers by GLC [Varian CP Wax 52CB column $\left(30 \mathrm{~m} \times 0.25 \mathrm{~mm}\right.$ ), flow rate $0.6 \mathrm{~mL} / \mathrm{min}$, method: $80^{\circ} \mathrm{C}$ for $2.00 \mathrm{~min}$, ramp @ 5.00 ${ }^{\circ} \mathrm{C} / \mathrm{min}$ to $250{ }^{\circ} \mathrm{C}$, hold for $20.00 \mathrm{~min}, \mathrm{~T}_{\mathrm{r}}=69.8$ (syn, E), 70.1 (anti, E) and 70.4 (anti, Z) min] provided the diastereomer ratio: 12.8:89.4:3.8. Separating the enantiomers by chiral HPLC Daicel Chiracel ${ }^{\mathrm{TM}}$ OD-H column, flow rate $0.5 \mathrm{ml} / \mathrm{min}, 4.0 \% \mathrm{i}-\mathrm{PrOH}, 96.0 \%$ hexanes, $\mathrm{T}_{\mathrm{r}} 10.6(2 R, 3 S)$ and $14.1(2 S, 3 R)$ provided the enantiomer ratio: $(2 R, 3 S)-8 \mathbf{e}^{\prime}:(2 S, 3 R)-8 \mathbf{e}^{\prime}=96: 4(92 \%$ ee $) . \quad[\alpha]_{\mathrm{D}}=+11.2\left(\right.$ c $\left.2.51, \mathrm{CHCl}_{3}\right) ;{ }^{1} \mathrm{H} \mathrm{NMR}\left(300 \mathrm{MHz}, \mathrm{CDCl}_{3}\right): \delta$ 7.18-7.32 (m, 5H), $5.90(\mathrm{ddt}, \mathrm{J}=15.1,10.0,1.2 \mathrm{~Hz}, 1 \mathrm{H}), 5.50(\mathrm{dt}, \mathrm{J}=15.1,6.3 \mathrm{~Hz}, 1 \mathrm{H}), 3.61(\mathrm{~d}, \mathrm{~J}=10.0,1 \mathrm{H}), 3.46$ $(\mathrm{dd}, \mathrm{J}=11.6,5.6 \mathrm{~Hz}, 1 \mathrm{H}), 3.36(\mathrm{dd}, \mathrm{J}=11.6,6.7 \mathrm{~Hz}, 1 \mathrm{H}), 2.05(\mathrm{qd}, \mathrm{J}=7.4,6.3 \mathrm{~Hz}, 2 \mathrm{H}), 1.10-1.80(\mathrm{~m}, 11 \mathrm{H}), 0.99$ $(\mathrm{t}, \mathrm{J}=7.4 \mathrm{~Hz}, 3 \mathrm{H}), 0.84(\mathrm{~s}, 3 \mathrm{H}) \mathrm{ppm} ;{ }^{13} \mathrm{C} \mathrm{NMR}\left(75 \mathrm{MHz}, \mathrm{CDCl}_{3}\right): \delta 143.37,134.17,129.23,128.77,128.19$, 126.17, 66.90, 52.40, 43.44, 42.97, 27.98, 27.87, 27.50, 27.40, 26.88, 25.82, 16.82, 13.91 ppm; IR (liquid film): 3467, 2959, 1738, 1451, 1375, 1265, 1032, 973, 739, $704 \mathrm{~cm}^{-1}$; MS (EI, $\left.70 \mathrm{eV}\right): \mathrm{m} / \mathrm{z} 268\left(\mathrm{M}^{+}-\mathrm{H}_{2} \mathrm{O}\right), 255,146,123$, 117; HRMS $m / z$ calcd for $\mathrm{C}_{20} \mathrm{H}_{28}\left(\mathrm{M}^{+}-\mathrm{H}_{2} \mathrm{O}\right): 268.2191$; found 268.2197.

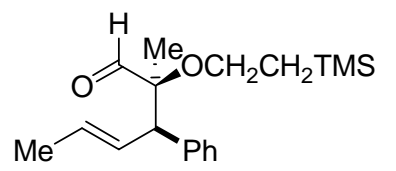

anti-(E)-2-Methyl-3-phenyl-2-[2-(trimethylsilyl)ethoxy]hex-4-enal (8f): General Procedure $\mathbf{C}$ was followed employing diallyl ether (7f, $152 \mathrm{mg}, 0.5 \mathrm{mmol})$ and $4 \mathrm{~mol} \%$ catalyst 3 (9.0 $\mathrm{mg}\left[\mathrm{IrCl}\left(\mathrm{C}_{8} \mathrm{H}_{14}\right)_{2}\right]_{2}, 16.8 \mathrm{mg} \mathrm{PCy}_{3}$, and $\left.6.8 \mathrm{mg} \mathrm{NaBPh}_{4}\right)$ and stirred $60 \mathrm{~h}$ at $80{ }^{\circ} \mathrm{C}$ in 1,2-dichloroethane solvent; anti:syn $=94: 6$. Purifying the crude product mixture by flash chromatography on silica gel $\left(2.5 \% \mathrm{Et}_{2} \mathrm{O}\right.$ in hexanes) yielded $88 \mathrm{mg}(58 \%)$ of the title compound as a light yellow oil. ${ }^{1} \mathrm{H}$ NMR (300 MHz, $\left.\mathrm{CDCl}_{3}\right): \delta 9.77(\mathrm{~s}, 1 \mathrm{H}), 6.00(\mathrm{ddq}, \mathrm{J}=15.2,9.4,1.6 \mathrm{~Hz}, 1 \mathrm{H}), 5.47(\mathrm{dq}, \mathrm{J}=15.2,6.5 \mathrm{~Hz}$, $1 \mathrm{H}), 3.57(\mathrm{td}, \mathrm{J}=9.1,6.4 \mathrm{~Hz}, 1 \mathrm{H}), 3.43(\mathrm{td}, \mathrm{J}=9.1,6.7 \mathrm{~Hz}, 1 \mathrm{H}), 3.39(\mathrm{~d}, \mathrm{~J}=9.4 \mathrm{~Hz}, 1 \mathrm{H}), 1.68(\mathrm{dd}, \mathrm{J}=6.4,1.6 \mathrm{~Hz}$, $3 \mathrm{H}), 1.13(\mathrm{~s}, 3 \mathrm{H}), 0.96-1.08(\mathrm{~m}, 2 \mathrm{H}), 0.07$ (s, 9H) ppm; ${ }^{13} \mathrm{C} \mathrm{NMR}\left(75 \mathrm{MHz}, \mathrm{CDCl}_{3}\right): \delta 208.0,140.2,129.3,129.2$, 128.0, 127.9, 126.7, 84.0, 61.8, 56.8, 18.9, 17.9, 16.7, -1.3 ppm; IR (liquid film): 2953, 1732, 1452, 1378, 1249, 1056, 970, 860, 837, $700 \mathrm{~cm}^{-1}$. MS (EI, $\left.70 \mathrm{eV}\right): \mathrm{m} / \mathrm{z}, 276\left(\mathrm{M}-\mathrm{C}_{2} \mathrm{H}_{4}^{+}\right), 219,186,157,147,131,115,91,84,73$; HRMS m/z calcd for $\mathrm{C}_{16} \mathrm{H}_{24} \mathrm{O}_{2} \mathrm{Si}\left(\mathrm{M}^{+}\right)$: 276.1546; found: 276.1548 .

syn-(E)-2-Ethyl-2,3-dimethylnon-4-enal (8g): General Procedure $\mathbf{C}$ was followed employing
$(2 E)-4-[(E)-2-m e t h y l b u t-2-e n y l o x y]$ oct-2-ene $(7 \mathbf{g}, 196 \mathrm{mg}, 1.0 \mathrm{mmol})$ and $2 \mathrm{~mol} \%$ catalyst $3(9.0$ 
$\mathrm{Et}_{2} \mathrm{O}$ in hexanes) yielded $147 \mathrm{mg}(75 \%)$ of the title compound as a colorless oil (syn:anti $\left.=95: 5\right) . \quad{ }^{1} \mathrm{H}$ NMR (300 $\left.\mathrm{MHz}, \mathrm{CDCl}_{3}\right): \delta 9.45(\mathrm{~s}, 1 \mathrm{H}), 5.43(\mathrm{dt}, \mathrm{J}=15.2,6.4 \mathrm{~Hz}, 1 \mathrm{H}), 5.27(\mathrm{dd}, \mathrm{J}=15.2,8.4 \mathrm{~Hz}, 1 \mathrm{H}), 2.39(\mathrm{dq}, \mathrm{J}=8.4,6.9$ $\mathrm{Hz}, 1 \mathrm{H}), 2.34-2.44(\mathrm{~m}, 1 \mathrm{H}), 1.94-2.00(\mathrm{~m}, 2 \mathrm{H}), 1.27-1.73(\mathrm{~m}, 6 \mathrm{H}), 0.97(\mathrm{~d}, \mathrm{~J}=6.9 \mathrm{~Hz}, 3 \mathrm{H}), 0.94(\mathrm{~s}, 3 \mathrm{H}), 0.88(\mathrm{t}, \mathrm{J}$ $=7.4 \mathrm{~Hz}, 3 \mathrm{H}), 0.80(\mathrm{t}, \mathrm{J}=7.5 \mathrm{~Hz}, 3 \mathrm{H}) \mathrm{ppm} ;{ }^{13} \mathrm{C}\left(75 \mathrm{MHz}, \mathrm{CDCl}_{3}\right): 207.3,132.0,130.9,51.9,41.6,32.2,31.6,26.6$, 22.1, 14.8, 14.2, 13.9, 8.4 ppm; MS (EI, $70 \mathrm{eV}): \mathrm{m} / \mathrm{z} 196\left(\mathrm{M}^{+}\right), 181,139,110,81,71,69$; HRMS m/z calcd for $\mathrm{C}_{13} \mathrm{H}_{24} \mathrm{O}\left(\mathrm{M}^{+}\right)$: 196.1827; found 196.1820 .

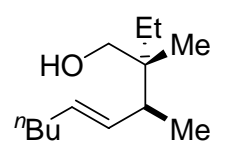

syn-(E)-2-Ethyl-2,3-dimethylnon-4-enol $\left(\mathbf{8 g} \mathbf{g}^{\prime}\right)$ : A $1 \mathrm{M}$ hexanes solution of ${ }^{i} \mathrm{Bu}_{2} \mathrm{AlH}(0.5 \mathrm{~mL}, 0.5$ mmol) was added to a $-78{ }^{\circ} \mathrm{C}$ solution of aldehyde $8 \mathrm{~g}(49 \mathrm{mg}, 0.25 \mathrm{mmol})$ in $\mathrm{CH}_{2} \mathrm{Cl}_{2}$. After stirring $30 \mathrm{~min}$ at $-78{ }^{\circ} \mathrm{C}$, a saturated aqueous sodium tartrate solution $(2 \mathrm{~mL})$ was added and the mixture was warmed to ambient temperature and stirred until two homogeneous phases formed $(\sim 1 \mathrm{~h})$. The mixture was extracted with $\mathrm{Et}_{2} \mathrm{O}(3 \times)$ and the combined organic extracts were dried $\left(\mathrm{MgSO}_{4}\right)$, concentrated, and the resulting crude oil was purified by flash chromatography on silica gel (16\% EtOAc in hexanes) yielded $29 \mathrm{mg}$ (60 $\%$ ) of the title compound as a light yellow oil. ${ }^{1} \mathrm{H}$ NMR $\left(300 \mathrm{MHz}, \mathrm{CDCl}_{3}\right): \delta 5.41-5.45(\mathrm{~m}, 2 \mathrm{H}), 3.41(\mathrm{~s}, 2 \mathrm{H})$, 2.11-2.21 (m, 1H), 1.97-2.01 (m, 2H), 1.37 (q, J = 7.6 Hz, H), 1.26-1.36 (m, 4H), 0.97 (d, J = 6.9 Hz, 3H), 0.94 (s, $3 \mathrm{H}), 0.88(\mathrm{t}, \mathrm{J}=7.4 \mathrm{~Hz}, 3 \mathrm{H}), 0.80(\mathrm{t}, \mathrm{J}=7.5 \mathrm{~Hz}, 3 \mathrm{H}) \mathrm{ppm} ;{ }^{13} \mathrm{C} \mathrm{NMR}\left(75 \mathrm{MHz}, \mathrm{CDCl}_{3}\right): \delta 133.2,130.5,68.0,41.4$, 39.7, 32.3, 31.8, 27.5, 22.2, 17.4, 15.2, 13.9, 7.9 ppm; MS (EI, $70 \mathrm{eV}): \mathrm{m} / \mathrm{z} 198\left(\mathrm{M}^{+}\right), 180,167,111,83,69$; HRMS $\mathrm{m} / \mathrm{z}$ calcd for $\mathrm{C}_{13} \mathrm{H}_{26} \mathrm{O}\left(\mathrm{M}^{+}\right)$: 198.1984 ; found 198.1975 .

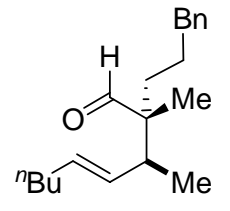

syn-(E)-2,3-Dimethyl-2-(3-phenylpropyl)non-4-enal (8h): General Procedure $\mathrm{C}$ was followed employing 1- $\{(3 E)-5-[(E)$-oct-2-en-4-yloxy]-4-methylpent-3-enyl $\}$ benzene (7h, $143 \mathrm{mg}, 0.5 \mathrm{mmol})$ and $2 \mathrm{~mol} \%$ catalyst $3\left(4.5 \mathrm{mg}\left[\operatorname{IrCl}\left(\mathrm{C}_{8} \mathrm{H}_{14}\right)_{2}\right]_{2}, 8.4 \mathrm{mg} \mathrm{PCy}\right.$ and $\left.3.4 \mathrm{mg} \mathrm{NaBPh}_{4}\right)$ and stirred $60 \mathrm{~h}$ at $75{ }^{\circ} \mathrm{C}$ in 1,2-dichloroethane solvent. Purifying the crude product mixture by flash chromatography on silica gel $\left(2.5 \% \mathrm{Et}_{2} \mathrm{O}\right.$ in hexanes) yielded $102 \mathrm{mg}(71 \%)$ of the title compound as a colorless oil (syn:anti = 95:5). Separating the stereoisomers by GLC [Varian CP Wax 52CB column ( $30 \mathrm{~m} \times 0.25 \mathrm{~mm}$ ), flow rate $0.6 \mathrm{~mL} / \mathrm{min}$, method: $80{ }^{\circ} \mathrm{C}$ for $2.00 \mathrm{~min}$, ramp @ $5.00{ }^{\circ} \mathrm{C} / \mathrm{min}$ to $250{ }^{\circ} \mathrm{C}$, hold for $20.00 \mathrm{~min}, \mathrm{~T}_{\mathrm{r}}=60.1$ and $61.0 \mathrm{~min}]$ provided the diastereomer ratio: anti:syn $=5.8: 94.2 .{ }^{1} \mathrm{H}$ NMR $\left(300 \mathrm{MHz}, \mathrm{CDCl}_{3}\right): \delta 9.45(\mathrm{~s}, 1 \mathrm{H})$, 7.15-7.31 (m, 5H), 5.42 (dt, J = 15.2, $6.5 \mathrm{~Hz}, 1 \mathrm{H}), 5.26(\mathrm{dd}, \mathrm{J}=15.2,8.5 \mathrm{~Hz}, 1 \mathrm{H}), 2.57-2.61$ (m, 2H), 2.24-2.48 (m, $2 \mathrm{H}), 1.93-1.98(\mathrm{~m}, 3 \mathrm{H}), 1.24-1.75(\mathrm{~m}, 6 \mathrm{H}), 0.96(\mathrm{~s}, 3 \mathrm{H}), 0.96(\mathrm{~d}, \mathrm{~J}=7.0 \mathrm{~Hz}, 3 \mathrm{H}), 0.89(\mathrm{t}, \mathrm{J}=7.1 \mathrm{~Hz}, 3 \mathrm{H}) \mathrm{ppm} ;{ }^{13} \mathrm{C}$ $\operatorname{NMR}\left(75 \mathrm{MHz}, \mathrm{CDCl}_{3}\right): \delta 207.1,142.0,132.2,130.7,128.32,128.25,125.8,51.6,41.8,36.5,33.7,32.2,31.6,29.7$, 25.9, 22.1, 14.9, 13.9 ppm; MS (EI, 70 eV): m/z $286\left(\mathrm{M}^{+}\right), 258,256,245,229,192,163,145,117,111,104,91,69$; HRMS $m / z$ calcd for $\mathrm{C}_{20} \mathrm{H}_{30} \mathrm{O}\left(\mathrm{M}^{+}\right) ; 286.2297$; found 286.2288 .

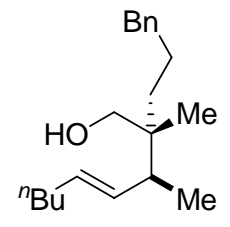

syn-(E)-2,3-Dimethyl-2-(3-phenylpropyl)non-4-enol (8h'): A $1 \mathrm{M}$ hexanes solution of ${ }^{i} \mathrm{Bu}_{2} \mathrm{AlH}$ $(0.5 \mathrm{~mL}, 0.5 \mathrm{mmol})$ was added to a $-78{ }^{\circ} \mathrm{C}$ solution of aldehyde $8 \mathbf{h}(72 \mathrm{mg}, 0.25 \mathrm{mmol})$ in $\mathrm{CH}_{2} \mathrm{Cl}_{2}$. After stirring $30 \mathrm{~min}$ at $-78{ }^{\circ} \mathrm{C}$, a saturated aqueous sodium tartrate solution $(2 \mathrm{~mL})$ was added and the mixture was warmed to ambient temperature and stirred until two homogeneous phases formed $(\sim 1 \mathrm{~h})$. The mixture was extracted with $\mathrm{Et}_{2} \mathrm{O}(3 \times)$ and the combined organic extracts were dried $\left(\mathrm{MgSO}_{4}\right)$, concentrated, and the resulting crude oil was purified by flash chromatography on silica gel (16\% EtOAc in hexanes) yielded $65 \mathrm{mg}(90 \%)$ of the title compound as a light yellow oil. Separating the stereoisomers by GLC [Varian CP Wax 52CB column $(30 \mathrm{~m} \times 0.25 \mathrm{~mm})$, flow rate $0.6 \mathrm{~mL} / \mathrm{min}$, method: $80^{\circ} \mathrm{C}$ for $2.00 \mathrm{~min}$, ramp @ 5.00 ${ }^{\circ} \mathrm{C} / \mathrm{min}$ to $250{ }^{\circ} \mathrm{C}$, hold for $20.00 \mathrm{~min}, \mathrm{~T}_{\mathrm{r}}=64.6$ and $68.9 \mathrm{~min}$ ] provided the diastereomer ratio: ANTI:SYN $=$ 
3.7:96.3; ${ }^{1} \mathrm{H}$ NMR $\left(300 \mathrm{MHz}, \mathrm{CDCl}_{3}\right): \delta 7.15-7.31(\mathrm{~m}, 5 \mathrm{H}), 5.39-5.41(\mathrm{~m}, 2 \mathrm{H}), 3.41(\mathrm{~d}, \mathrm{~J}=11.4 \mathrm{~Hz}, 1 \mathrm{H}), 3.37(\mathrm{~d}, \mathrm{~J}$ $=11.4 \mathrm{~Hz}, 1 \mathrm{H}), 2.58(\mathrm{t}, \mathrm{J}=7.7 \mathrm{~Hz}, 2 \mathrm{H}), 2.12-2.19(\mathrm{~m}, 1 \mathrm{H}), 1.95-2.05(\mathrm{~m}, 2 \mathrm{H}), 1.52-1.74(\mathrm{~m}, 3 \mathrm{H}), 1.26-1.46(\mathrm{~m}$, $6 \mathrm{H}), 0.90(\mathrm{~d}, \mathrm{~J}=6.9 \mathrm{~Hz}, 3 \mathrm{H}), 0.89(\mathrm{t}, \mathrm{J}=7.4 \mathrm{~Hz}, 3 \mathrm{H}), 0.75(\mathrm{~s}, 3 \mathrm{H}) \mathrm{ppm} ;{ }^{13} \mathrm{C} \mathrm{NMR}\left(75 \mathrm{MHz}, \mathrm{CDCl}_{3}\right): \delta 143.0,133.4$, 130.9, 128.7, 128.6, 126.0, 68.8, 42.0, 40.1, 37.2, 35.5, 32.6, 32.1, 25.8, 22.5, 18.4, 15.5, 14.2 ppm; MS (EI, 70 eV): $\mathrm{m} / \mathrm{z} 288\left(\mathrm{M}^{+}\right), 270,257,176,159,145,117,104,91$; HRMS $\mathrm{m} / \mathrm{z}$ calcd for $\mathrm{C}_{20} \mathrm{H}_{32} \mathrm{O}\left(\mathrm{M}^{+}\right): 288.2453$; found 288.2459.

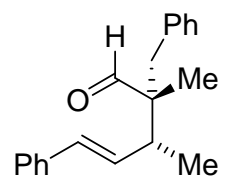

(2S,3R,E)-2-Benzyl-2,3-dimethyl-5-phenylpent-4-enal (15): General Procedure $C$ was followed employing 1-(2-\{[(R)-1-phenylbut-3-enyloxy]methyl $\}$ allyl)benzene [7g (91\% ee), $139 \mathrm{mg}, 0.5$ mmol] and $2 \mathrm{~mol} \%$ catalyst $3\left(4.5 \mathrm{mg}\left[\operatorname{IrCl}\left(\mathrm{C}_{8} \mathrm{H}_{14}\right)_{2}\right]_{2}, 8.4 \mathrm{mg} \mathrm{PCy}\right.$, and $3.4 \mathrm{mg} \mathrm{NaBPh}$ ) and stirred $60 \mathrm{~h}$ at $60{ }^{\circ} \mathrm{C}$ in 1,2-dichloroethane solvent. The crude product mixture was purified by flash chromatography on silica gel $\left(2.5 \% \mathrm{Et}_{2} \mathrm{O}\right.$ in hexanes) to yield $101 \mathrm{mg}(73 \%)$ of the title compound as colorless oil: anti:syn > 98: 2). Separating the enantiomers by chiral HPLC Daicel Chiracel ${ }^{\mathrm{TM}}$ OD-H column, flow rate 0.8 $\mathrm{mL} / \mathrm{min}, 0.8 \% \mathrm{i}-\mathrm{PrOH}, 99.2 \%$ hexanes, $\mathrm{T}_{\mathrm{r}} 30.55(2 R, 3 S)$ and $36.09(2 S, 3 R)$ provided the enantiomer ratio: (2R, $3 S):(2 S, 3 R)=5.1: 94.9(90 \%$ ee $) . \quad[\alpha]_{\mathrm{D}}=-74.6\left(\mathrm{c} 5.09, \mathrm{CHCl}_{3}\right) ;{ }^{1} \mathrm{H} \mathrm{NMR}\left(300 \mathrm{MHz}, \mathrm{CDCl}_{3}\right): \delta 9.68(\mathrm{~s}, 1 \mathrm{H})$, 7.06-7.42 (m, 10H), $6.53(\mathrm{~d}, \mathrm{~J}=15.8 \mathrm{~Hz}, 1 \mathrm{H}), 6.19(\mathrm{dd}, \mathrm{J}=15.8,9.1 \mathrm{~Hz}, 1 \mathrm{H}), 3.09(\mathrm{~d}, \mathrm{~J}=13.8 \mathrm{~Hz}, 1 \mathrm{H}), 2.77(\mathrm{~d}, \mathrm{~J}$ $=13.8 \mathrm{~Hz}, 1 \mathrm{H}), 2.74(\mathrm{dq}, \mathrm{J}=9.1,6.9 \mathrm{~Hz}, 1 \mathrm{H}), 1.11(\mathrm{~d}, \mathrm{~J}=6.9 \mathrm{~Hz}, 3 \mathrm{H}), 1.02(\mathrm{~s}, 3 \mathrm{H}) \mathrm{ppm} ;{ }^{13} \mathrm{C} \mathrm{NMR}(75 \mathrm{MHz}$, $\left.\mathrm{CDCl}_{3}\right): \delta 206.6,137.0,136.8,132.0,130.2,128.6,128.1,127.4,126.5,126.2,53.4,42.3,41.8,16.2,14.0$ ppm; MS (EI, $70 \mathrm{eV}): \mathrm{m} / \mathrm{z} 278\left(\mathrm{M}^{+}\right), 263,249,187,146,131,115,105,91,77,65 ; \mathrm{HRMS} \mathrm{m} / \mathrm{z}$ calcd for $\mathrm{C}_{20} \mathrm{H}_{22} \mathrm{O}\left(\mathrm{M}^{+}\right)$: 278.1671 ; found 278.1662 .

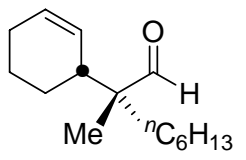

$R^{*}$-(R)-2-[(R)-Cyclohex-2-enyl]-2-methyloctanal (17): General Procedure $\mathbf{C}$ was followed employing diallyl ether 16a $(111 \mathrm{mg}, 0.5 \mathrm{mmol})$ and $4 \mathrm{~mol} \%$ catalyst $3\left(9.0 \mathrm{mg}\left[\operatorname{IrCl}\left(\mathrm{C}_{8} \mathrm{H}_{14}\right)_{2}\right]_{2}\right.$,

$16.8 \mathrm{mg} \mathrm{PCy}_{3}$, and $6.8 \mathrm{mg} \mathrm{NaBPh}_{4}$ ) and stirred $48 \mathrm{~h}$ at $80{ }^{\circ} \mathrm{C}$ in 1,2-dichloroethane. After cooling the reaction mixture to ambient temperature, the solvent was evaporated and the resulting residue was dissolved in 1,2-dichloroethane $(2 \mathrm{~mL})$ and cooled to $-40{ }^{\circ} \mathrm{C}$. A $1.0 \mathrm{M}$ hexanes solution of $\mathrm{Me}_{2} \mathrm{AlCl}(1.0 \mathrm{~mL}, 1.0$ $\mathrm{mmol}$ ) was added and the reaction was stirred for $10 \mathrm{~min}$. A saturated sodium tartrate solution $(4 \mathrm{~mL})$ was added and the mixture was warmed to ambient temperature and stirred until two homogeneous phases resulted $(\sim 1 \mathrm{~h})$. The mixture was extracted with $\mathrm{Et}_{2} \mathrm{O}(3 \times)$ and the combined organic extracts were dried over anhydrous $\mathrm{MgSO}_{4}$. Purifying the crude product mixture by flash chromatography on silica gel (2.5\% ethyl ether in hexanes) yielded 63 $\mathrm{mg}\left(57 \%, 100 \%\right.$ de) of the title compound as a light yellow oil. Analysis of the crude product mixture by ${ }^{1} \mathrm{H}$ NMR and GLC [Varian CP Wax 52CB column $\left(30 \mathrm{~m} \mathrm{x} 0.25 \mathrm{~mm}\right.$ ), flow rate $0.6 \mathrm{~mL} / \mathrm{min}$, method: $80{ }^{\circ} \mathrm{C}$ for 2.00 min, ramp@ $5.00{ }^{\circ} \mathrm{C} / \mathrm{min}$ to $250{ }^{\circ} \mathrm{C}$, hold for $20.00 \mathrm{~min}, \mathrm{~T}_{\mathrm{r}}=38.9 \mathrm{~min}$ ] provided evidence of only one diastereomer. ${ }^{1} \mathrm{H}$ NMR (300 MHz, $\left.\mathrm{CDCl}_{3}\right): \delta 9.49(\mathrm{~s}, 1 \mathrm{H}), 5.81(\mathrm{dq}, \mathrm{J}=10.3,3.1 \mathrm{~Hz}, 1 \mathrm{H}), 5.59(\mathrm{dt}, \mathrm{J}=10.3,3.5$ $\mathrm{Hz}, 1 \mathrm{H}), 2.38-2.48(\mathrm{~m}, 1 \mathrm{H}), 1.82-2.10(\mathrm{~m}, 2 \mathrm{H}), 1.20-1.90(\mathrm{~m}, 13 \mathrm{H}), 0.97(\mathrm{~s}, 3 \mathrm{H}), 0.88(\mathrm{t}, \mathrm{J}=7.4 \mathrm{~Hz}, 3 \mathrm{H}) ;{ }^{13} \mathrm{C} \mathrm{NMR}$ $\left(75 \mathrm{MHz}, \mathrm{CDCl}_{3}\right): \delta 207.1,130.0,126.2,40.4,33.8,31.6,30.0,25.1,24.5,24.0,22.6,22.3,14.8,14.1$ ppm; MS (EI, $70 \mathrm{ev}) \mathrm{m} / \mathrm{z} 222\left(\mathrm{M}^{+}\right), 207,193,179,142,137,81$; HRMS m/z calcd for $\mathrm{C}_{15} \mathrm{H}_{26} \mathrm{O}\left(\mathrm{M}^{+}\right)$: 222.1984; found 222.1977.

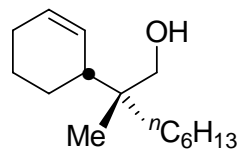

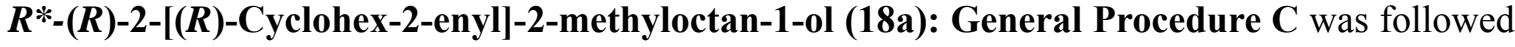
employing diallyl ether 16a $(111 \mathrm{mg}, 0.5 \mathrm{mmol})$ and $4 \mathrm{~mol} \%$ catalyst $\left(9.0 \mathrm{mg}\left[\operatorname{IrCl}\left(\mathrm{C}_{8} \mathrm{H}_{14}\right)_{2}\right]_{2}\right.$, $16.8 \mathrm{mg} \mathrm{PCy}$, and $6.8 \mathrm{mg} \mathrm{NaBPh}$ ) and stirred $48 \mathrm{~h}$ at $80{ }^{\circ} \mathrm{C}$ in 1,2-dichloroethane solvent. After cooling the reaction mixture to ambient temperature the solvent was evaporated and the 
resulting residue was dissolved in dichloromethane $(2 \mathrm{~mL})$ and cooled to $-78{ }^{\circ} \mathrm{C}$. A $1.0 \mathrm{M}$ hexanes solution of $i-\mathrm{Bu}_{2} \mathrm{AlH}(1.0 \mathrm{~mL}, 1.0 \mathrm{mmol})$ was added and the reaction was warmed to ambient temperature and stirred $12 \mathrm{~h}$. A saturated sodium tartrate solution $(4 \mathrm{~mL})$ was added and the mixture was stirred $1 \mathrm{~h}$. The mixture was extracted with $\mathrm{Et}_{2} \mathrm{O}(3 \times)$ and the combined organic extracts were dried over anhydrous $\mathrm{MgSO}_{4}$. Purifying the crude product mixture by flash chromatography on silica gel (16\% EtOAc in hexanes) yielded $78 \mathrm{mg}(71 \%)$ of the title compound as a light yellow oil. Separating the stereoisomers by GLC [Varian CP Wax 52CB column (30 m x $0.25 \mathrm{~mm}$ ), flow rate $0.6 \mathrm{~mL} / \mathrm{min}$, method: $80{ }^{\circ} \mathrm{C}$ for $2.00 \mathrm{~min}$, ramp $@ 5.00{ }^{\circ} \mathrm{C} / \mathrm{min}$ to $250{ }^{\circ} \mathrm{C}$, hold for $20.00 \mathrm{~min}, \mathrm{~T}_{\mathrm{r}}=47.1$ and $50.0 \mathrm{~min}$ ] provided the diastereomer ratio: anti:syn $=1.0: 99.0 .{ }^{1} \mathrm{H} \mathrm{NMR}\left(300 \mathrm{MHz}, \mathrm{CDCl}_{3}\right): \delta 5.69-5.81(\mathrm{~m}$, 2H), $3.54(\mathrm{~d}, \mathrm{~J}=11.1 \mathrm{~Hz}, 1 \mathrm{H}), 3.39(\mathrm{~d}, \mathrm{~J}=11.1 \mathrm{~Hz}, 1 \mathrm{H}), 2.15-2.25(\mathrm{~m}, 1 \mathrm{H}), 1.90-2.00(\mathrm{~m}, 2 \mathrm{H}), 1.65-1.88(\mathrm{~m}, 2 \mathrm{H})$, 1.20-1.62 (m, 13H), $0.89(\mathrm{t}, \mathrm{J}=6.5 \mathrm{~Hz}, 3 \mathrm{H}), 0.85(\mathrm{~s}, 3 \mathrm{H}) \mathrm{ppm} ;{ }^{13} \mathrm{C}$ NMR $\left(75 \mathrm{MHz}, \mathrm{CDCl}_{3}\right): \delta 128.70,128.64$, 68.21, 40.39, 39.70, 35.18, 31.89, 30.35, 25.22, 23.84, 23.35, 22.83, 22.69, 19.23, 14.09 ppm; MS (EI, 70 ev): 224 $\left(\mathrm{M}^{+}\right), 206,193,143,121,109$; HRMS m/z calcd for $\mathrm{C}_{15} \mathrm{H}_{28} \mathrm{O}\left(\mathrm{M}^{+}\right)$224.2140, found: 224.2131.

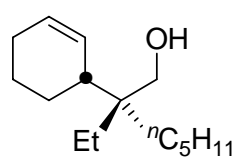

$R^{*}$-(R)-2-[(R)-Cyclohex-2-enyl]-2-methylheptan-1-ol (18b): General Procedure C was followed employing diallyl ether $16 \mathbf{b}(67 \mathrm{mg}, 0.3 \mathrm{mmol})$ and $4 \mathrm{~mol} \%$ catalyst $(5.4 \mathrm{mg}$ $\left[\operatorname{IrCl}\left(\mathrm{C}_{8} \mathrm{H}_{14}\right)_{2}\right]_{2}, \quad 10.1 \mathrm{mg} \mathrm{PCy}_{3}$, and $\left.4.1 \mathrm{mg} \mathrm{NaBPh}_{4}\right)$ and stirred $48 \mathrm{~h}$ at $80{ }^{\circ} \mathrm{C}$ in 1,2-dichloroethane solvent. After cooling the reaction mixture to ambient temperature, the solvents were evaporated and the resulting residue was dissolved in dichloromethane $(2 \mathrm{~mL})$. The reaction mixture was cooled to $-78{ }^{\circ} \mathrm{C}$ and a $1.0 \mathrm{M}$ hexanes solution of $i-\mathrm{Bu}_{2} \mathrm{AlH}(0.6 \mathrm{~mL}, 0.6 \mathrm{mmol})$ was added and the reaction was warmed to ambient temperature and stirred for $12 \mathrm{~h}$. A saturated sodium tartrate solution $(4 \mathrm{~mL})$ was added and the mixture was warmed to ambient temperature and stirred for $1 \mathrm{~h}$. The mixture was extracted with $\mathrm{Et}_{2} \mathrm{O}(3 \times)$ and the combined organic extracts were dried over anhydrous $\mathrm{MgSO}_{4}$. Purifying the crude product mixture by flash chromatography on silica gel (16\% EtOAc in hexanes) yielded $42 \mathrm{mg}(62 \%)$ of the title compound as a light yellow oil. Separating the stereoisomers by GLC [Varian CP Wax 52CB column (30 $\mathrm{m} \times 0.25 \mathrm{~mm})$, flow rate $0.6 \mathrm{~mL} / \mathrm{min}$, method: $80{ }^{\circ} \mathrm{C}$ for $2.00 \mathrm{~min}$, ramp @ $5.00{ }^{\circ} \mathrm{C} / \mathrm{min}$ to $250{ }^{\circ} \mathrm{C}$, hold for $20.00 \mathrm{~min}, \mathrm{~T}_{\mathrm{r}}=46.3$ and $48.8 \mathrm{~min}$ ] provided the diastereomer ratio: anti:syn $=3.8: 96.2 .{ }^{1} \mathrm{H} \mathrm{NMR}\left(300 \mathrm{MHz}, \mathrm{CDCl}_{3}\right): \delta 5.86(\mathrm{~d}, \mathrm{~J}=$ $10.4 \mathrm{~Hz}, 1 \mathrm{H}), 5.75(\mathrm{dq}, \mathrm{J}=10.4,3.2 \mathrm{~Hz}, 1 \mathrm{H}), 3.59(\mathrm{~d}, \mathrm{~J}=11.1 \mathrm{~Hz}, 1 \mathrm{H}), 3.39(\mathrm{~d}, \mathrm{~J}=11.1 \mathrm{~Hz}, 1 \mathrm{H}), 2.15-2.25(\mathrm{~m}$, $1 \mathrm{H}), 1.95-2.05(\mathrm{~m}, 2 \mathrm{H}), 1.72-1.89(\mathrm{~m}, 3 \mathrm{H}), 1.21-1.67(\mathrm{~m}, 12 \mathrm{H}), 0.89(\mathrm{t}, \mathrm{J}=7.5 \mathrm{~Hz}, 3 \mathrm{H}), 0.84(\mathrm{t}, \mathrm{J}=7.5 \mathrm{~Hz}, 3 \mathrm{H})$ ppm; ${ }^{13} \mathrm{C}$ NMR (75 MHz, $\left.\mathrm{CDCl}_{3}\right): \delta 129.30,128.69,67.19,42.18,39.94,32.94,31.79,31.58,25.34,24.26,23.99$, 22.96, 22.79, 14.12, 7.92 ppm; MS (EI, 70 ev): $224\left(\mathrm{M}^{+}\right)$, 206, 195, 193, 177, 163, 153, 149, 143, 142, 135, 125, 109, 95, 83, 69, 55; HRMS m/z calcd for $\mathrm{C}_{15} \mathrm{H}_{28} \mathrm{O}\left(\mathrm{M}^{+}\right)$: 224.2140; found: 224.2138 . 


\section{Appendix I}

\section{X-ray structure of semicarbazide 8a' derived from anti-2-[(E)-1,3-Diphenylallyl]-2-methylhexanal (8a)}

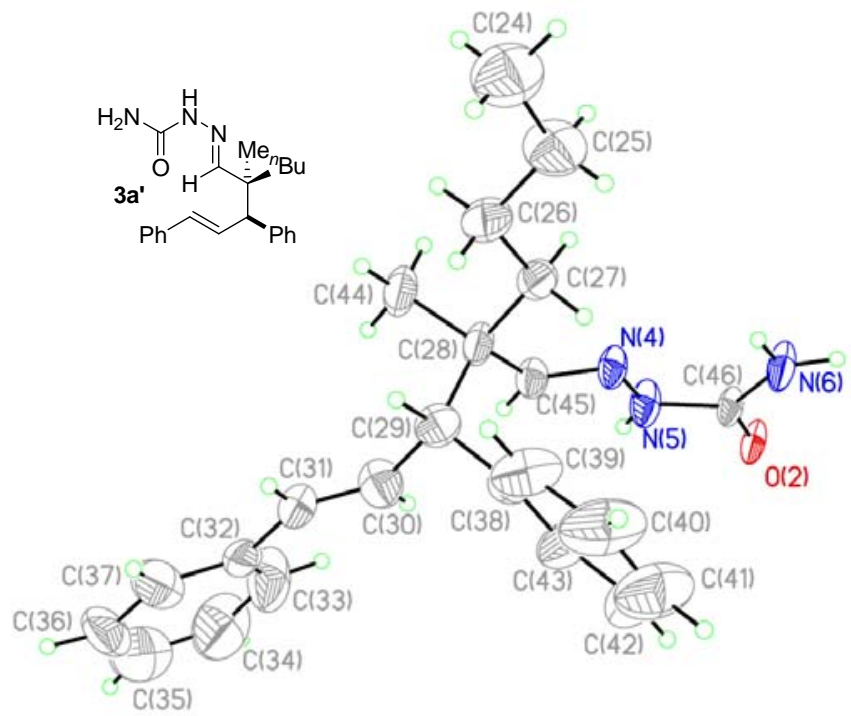

Figure sl-1. X-ray Crystal Structure of anti-(1E)-1-[(E)-2-Butyl-2-methyl-3,5-diphenylpent-4-enylidene]semicarbazide $\left(\mathbf{8 a}^{\prime}\right)$

Table S-1. Crystal data and structure refinement for $\mathbf{8 a}^{\prime}$

Empirical formula

Formula weight

Temperature

Wavelength

Crystal system

Space group

Unit cell dimensions

Volume

Z

Density (calculated)

Absorption coefficient

$\mathrm{F}(000)$

Crystal size

Theta range for data collection

Index ranges

Reflections collected

Independent reflections

Completeness to theta $=25.00^{\circ}$

Absorption correction

Max. and min. transmission

Refinement method

Data / restraints / parameters

Goodness-of-fit on $\mathrm{F}^{2}$

Final R indices [I $>2 \operatorname{sigma}(\mathrm{I})]$

$\mathrm{R}$ indices (all data)

Largest diff. peak and hole
$\mathrm{C}_{23} \mathrm{H}_{29} \mathrm{~N}_{3} \mathrm{O}$

363.49

295(2) K

$0.71073 \AA$

Triclinic

P-1

$\mathrm{a}=10.440(2) \AA$

$\alpha=114.083(4)^{\circ}$.

$\mathrm{b}=14.165(3) \AA$

$\beta=105.058(4)^{\circ}$.

$\mathrm{c}=16.869(4) \AA$

$2168.6(8) \AA^{3}$

4

$1.113 \mathrm{Mg} / \mathrm{m}^{3}$

$0.069 \mathrm{~mm}^{-1}$

784

$0.15 \times 0.08 \times 0.08 \mathrm{~mm}^{3}$

1.60 to $25.00^{\circ}$

$-12<=\mathrm{h}<=12,-16<=\mathrm{k}<=16,-20<=\mathrm{l}<=20$

15885

$7644[\mathrm{R}($ int $)=0.0905]$

$99.8 \%$

None

0.9945 and 0.9897

Full-matrix least-squares on $\mathrm{F}^{2}$

7644 / 0 / 511

1.111

$\mathrm{R} 1=0.1411, \mathrm{wR} 2=0.2873$

$\mathrm{R} 1=0.2571, \mathrm{wR} 2=0.3305$

0.272 and -0.229 e. $\AA^{-3}$ 


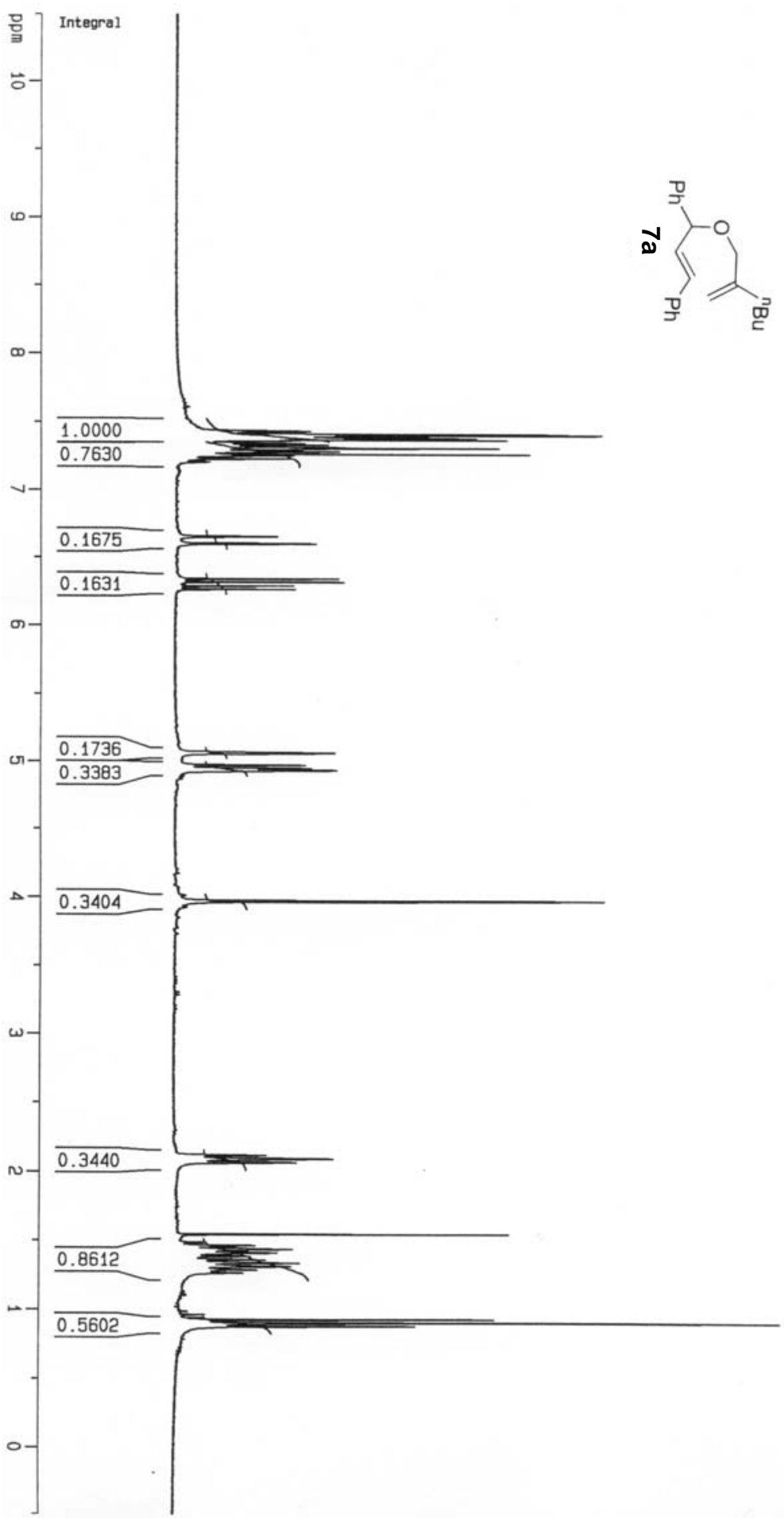

ppm

- 8.0991

- 7.6330

[ 7.4606

- 7.4042

||$\left[\begin{array}{r}7.3994 \\ -7.3909\end{array}\right.$

- 7.3871

- 7.3802

$-7.3759$

$-7.3728$

$-7.3641$

$-7.3258$

L 7.3091

- 7.3024

- 7.2964

$-7.2765$

- 7.2600

L 6.5975

- 6.3377

L 6.3145

- 6.2615

$-5.0628$

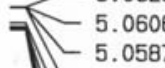

$-5.0565$

$-4.9689$

$-4.9452$

$-4.9344$

4.9308

L 4.9280

- 3.9696

- 3.9257

2.1170

F 2.0932

- 2.0669

- 1.5450

- 1.4638

$=\left[\begin{array}{l}1.4386 \\ -1.4348\end{array}\right.$

- 1.4119

$-1.4085$

1.3854

$-1.3582$

$-1.3367$

$-1.3342$

$-1.3104$

- 1.2850

- 1.2624

0.9231

$-0.8993$

$-0.8912$

0.8752 

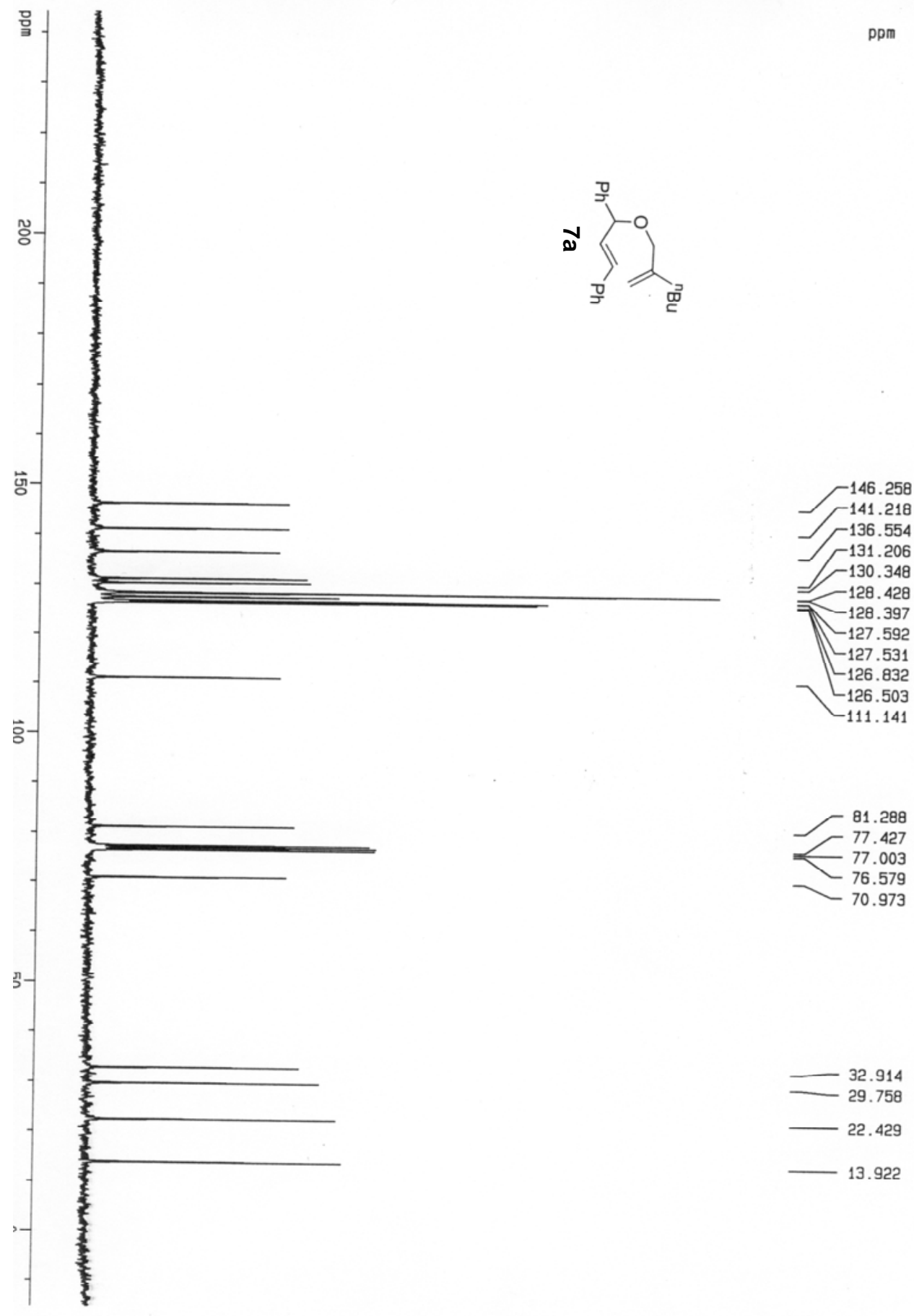

— 32.914

- 29.758

- 22.429

$-13.922$ 


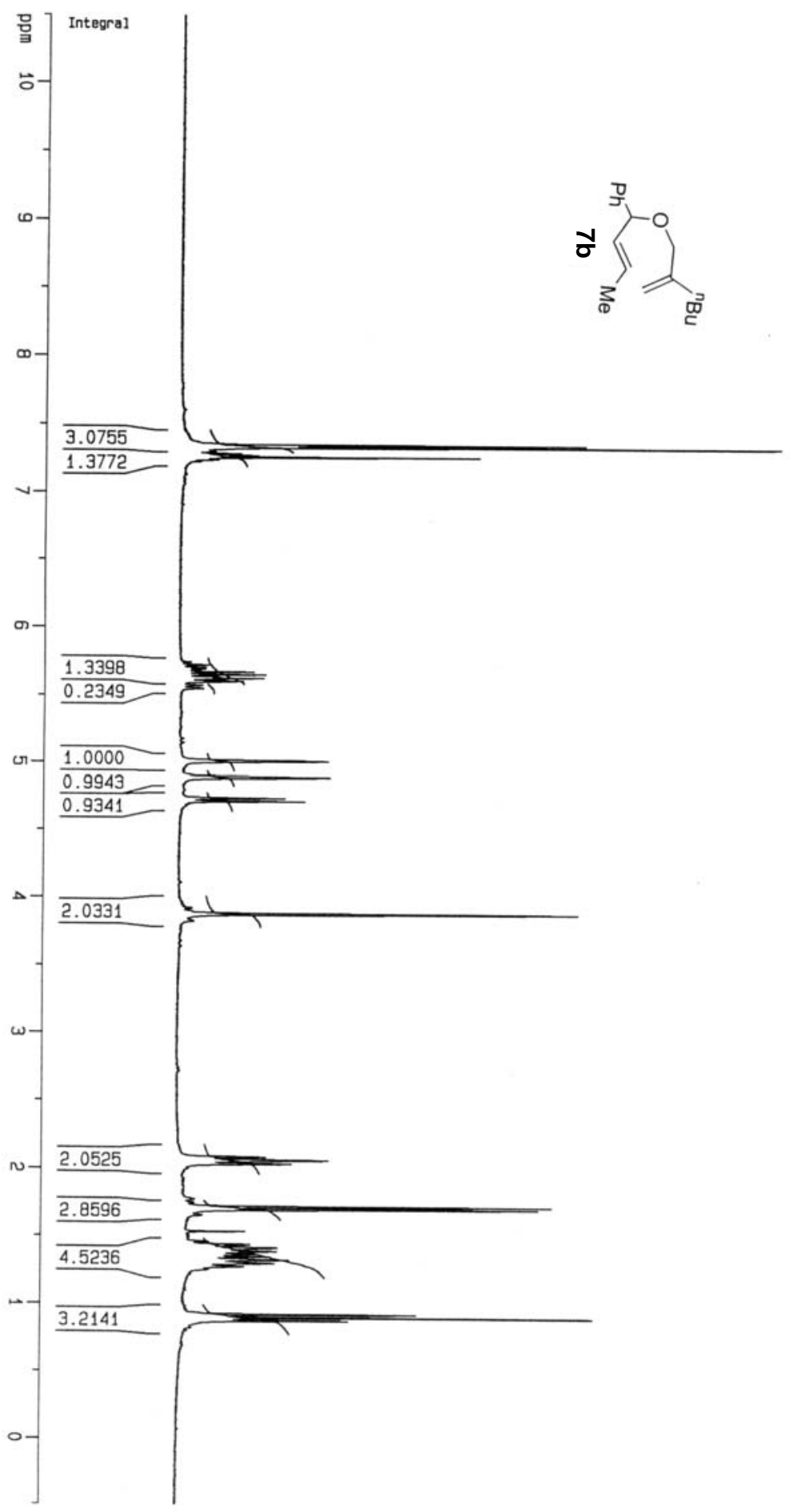

$-7.23792$

$-7.22493$

5.74170

5.72113

$-5.70159$

$-5.69063$

$-5.67028$

$-5.65077$

$-5.63234$

$-5.62285$

$-5.60238$

$-5.59944$

5.57132

] -5.54843

$-5.14578$

$-5.00748$

$-4.88756$

$-4.73047$

$-4.70812$

- $\begin{array}{r}-3.92141 \\ -3.87714\end{array}$

$-3.82563$

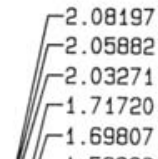

9807
3398

$\Gamma^{1.53390}$

$-1.43725$

$-1.42683$

$F_{-1.41285}^{1.42683}$

$-1.40001$

$-1.38520$

$-1.36264$

$-1.34431$

$-1.32000$

$-1.29598$

$-1.27096$

$-1.25047$

$-0.91559$

$-0.89155$

$-0.86807$ 

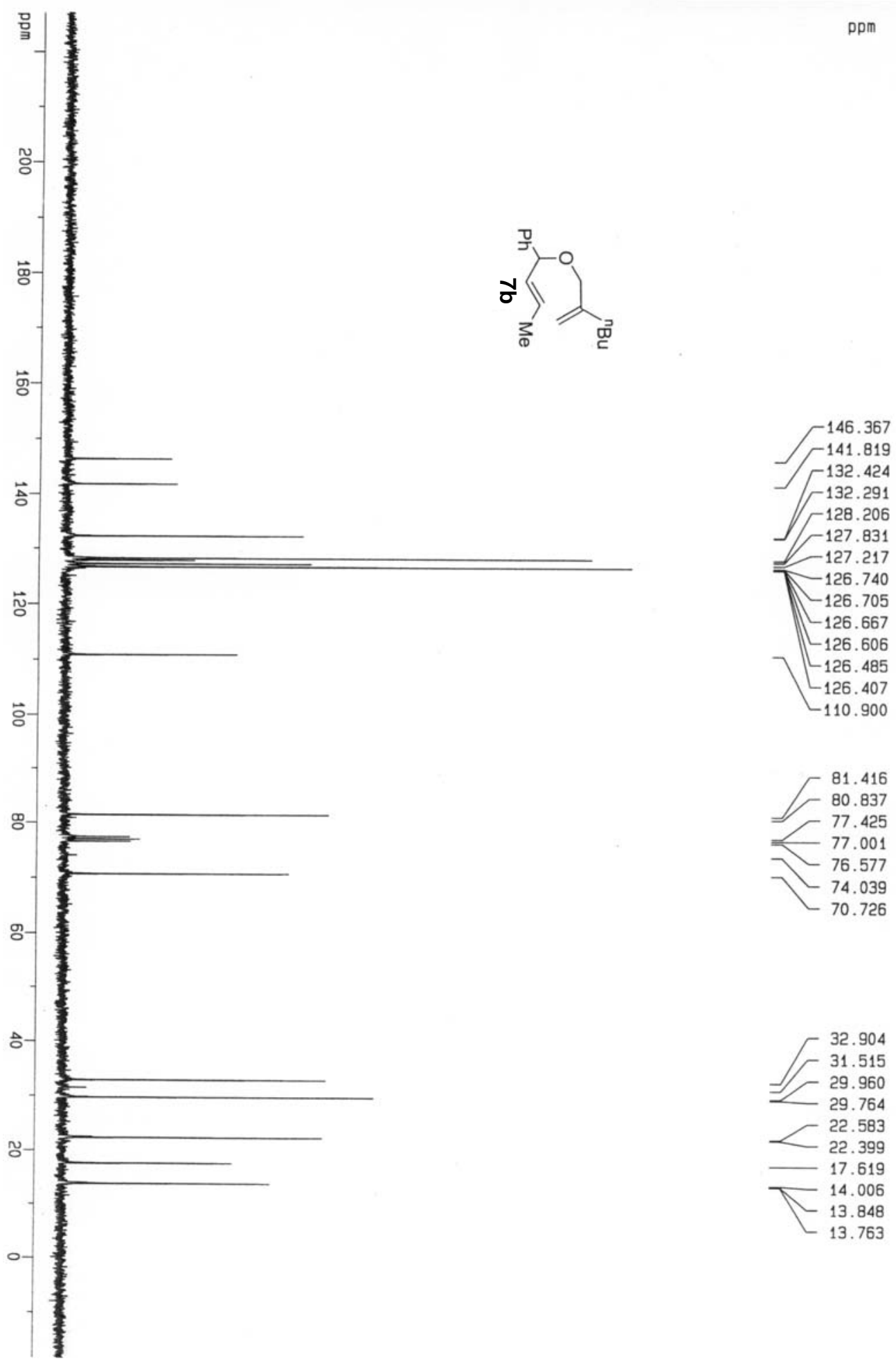


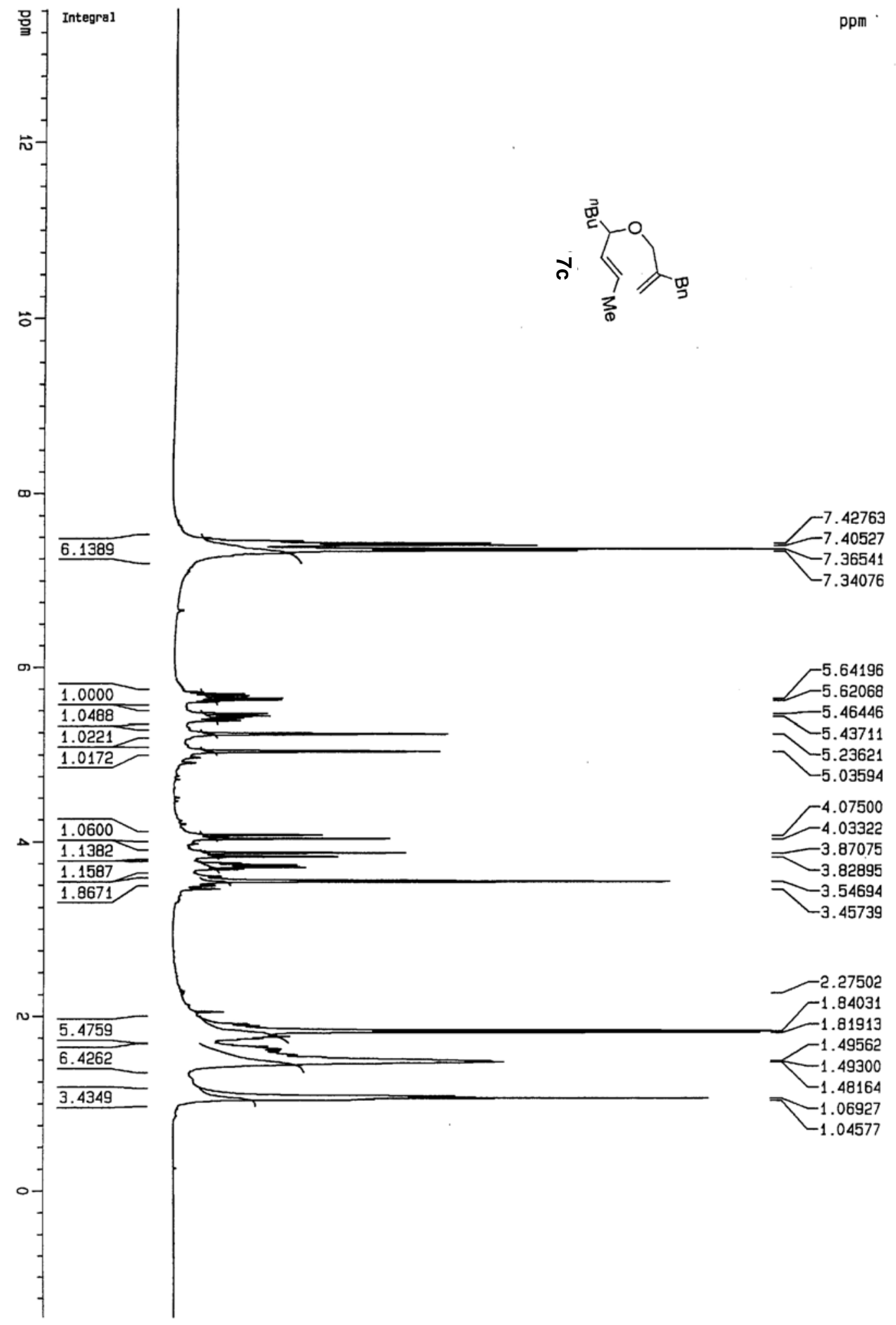

ppm 


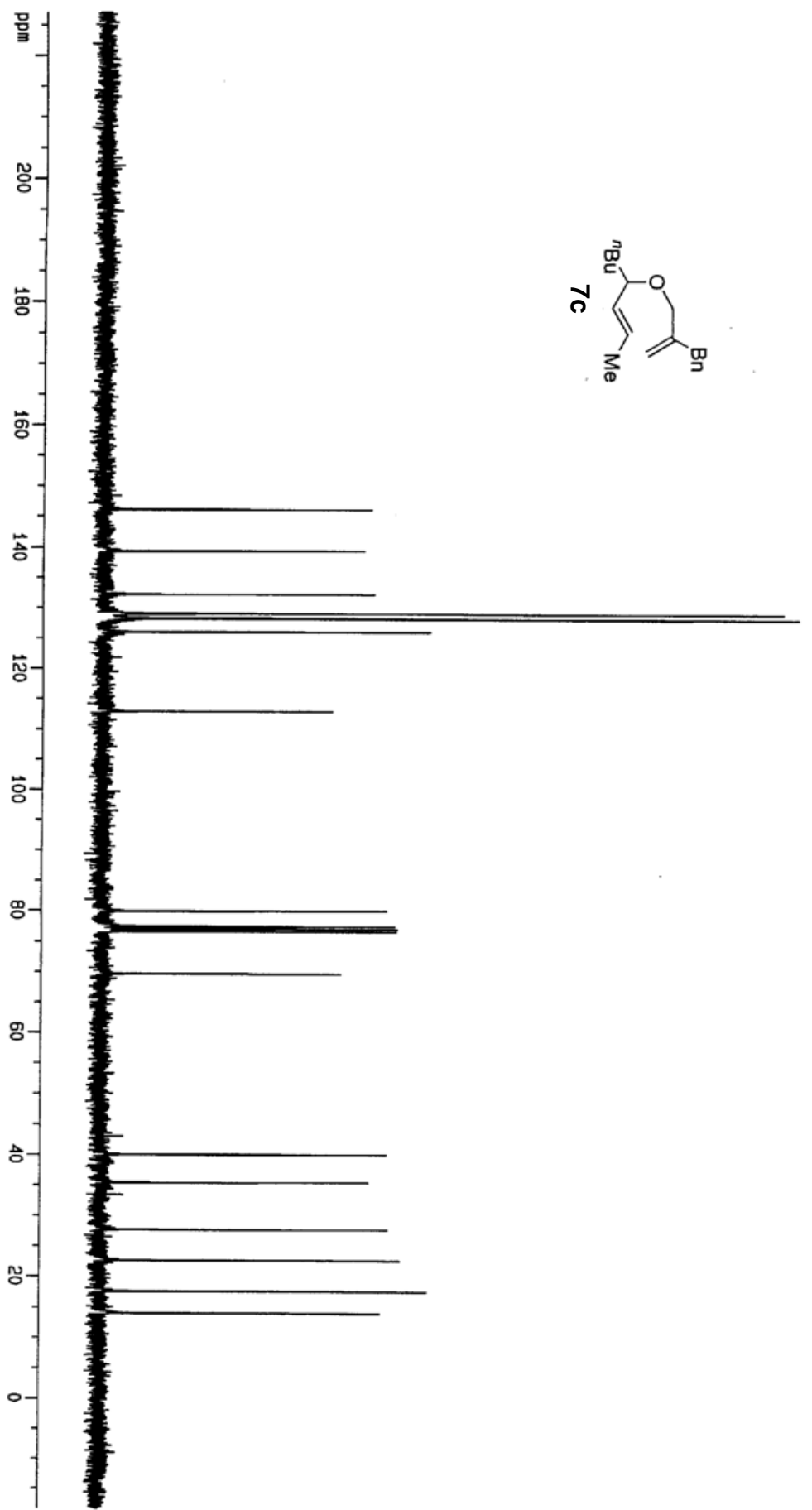

ppm

$-146.081$

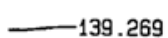

$-132.156$

$-128.983$

$-128.278$

$-128.142$

125.955

-112.859 


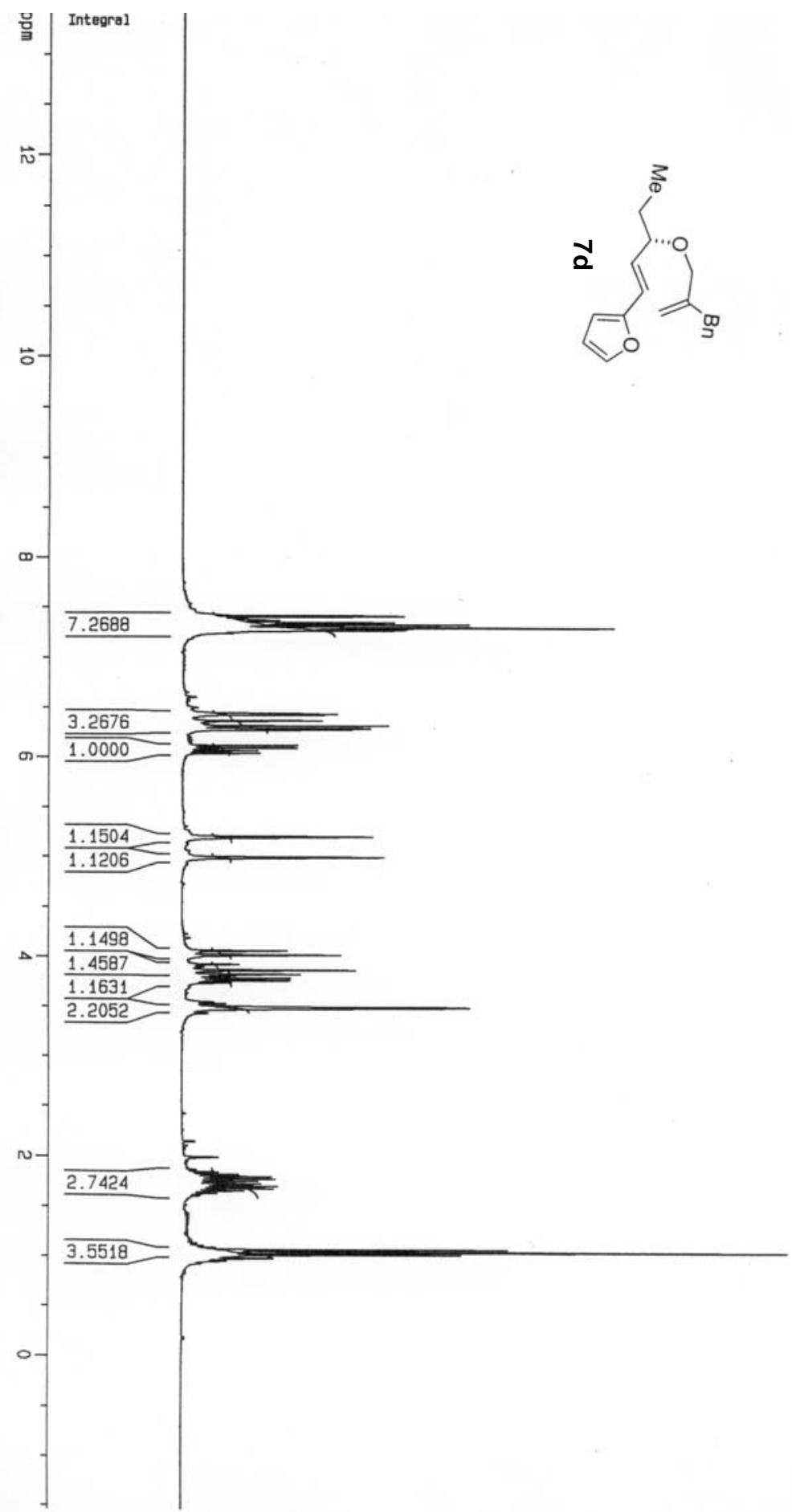

$-6.44040$

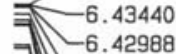

$-6.42988$

$-6.31254$

-6.28515

-6.27433

6.11399

6.08816

$-5.19318$

- -4.98885

$-4.05947$

$-4.01766$

-3.86601

$-3.82421$

-3.78563

$-3.76119$

$-3.48466$

$-1.80018$

$-1.77623$

$-1.75258$

$7-1.72693$

$-1.70278$

$L_{1} .68055$

$-1.36193$

$-1.28962$

] $L_{1.06473}$

$-1.04031$

$\leftarrow_{1.01537}$

$-0.97990$

- 0.80467

$-0.18988$

$L_{0.16886}$ 


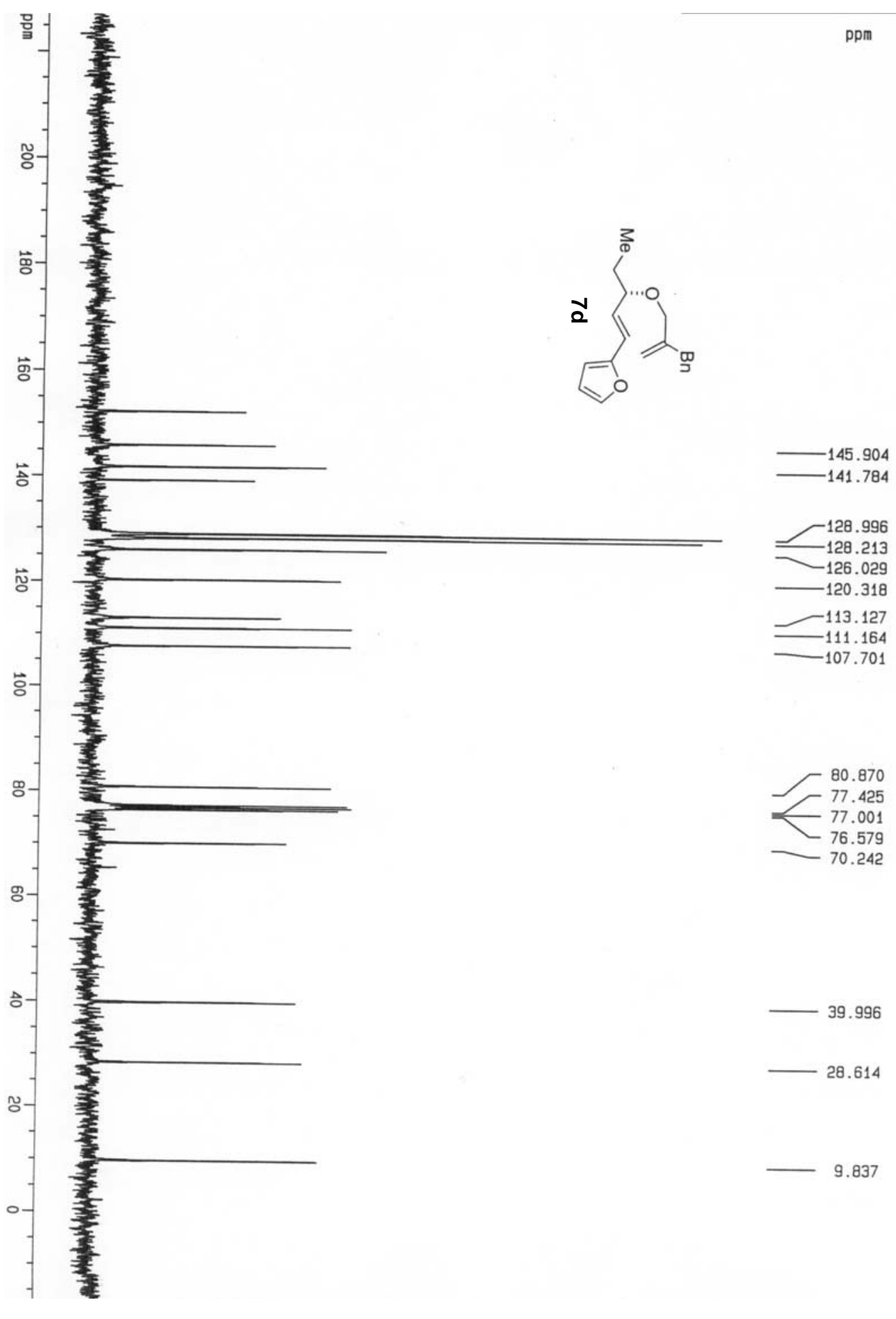




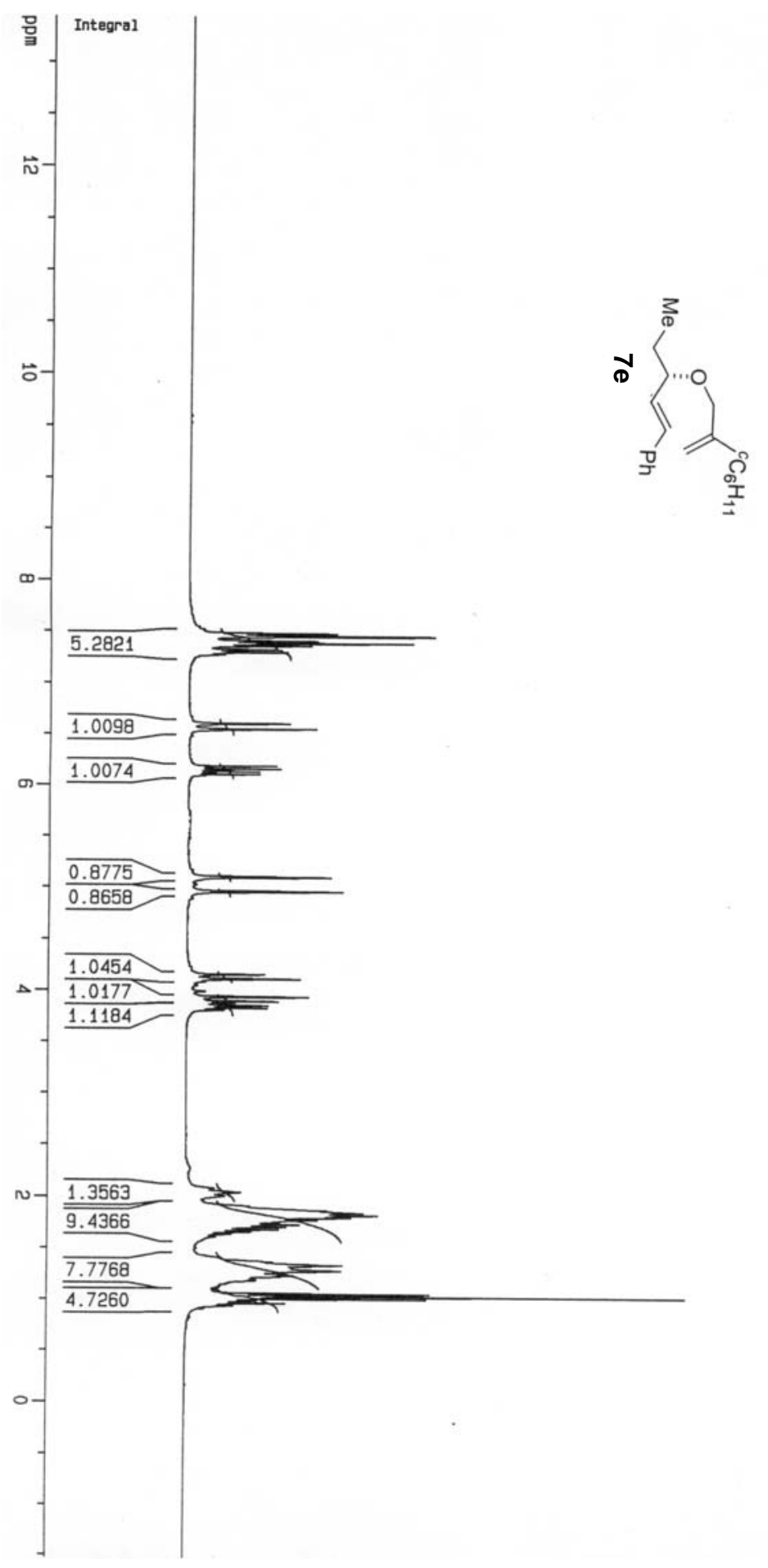

ppm

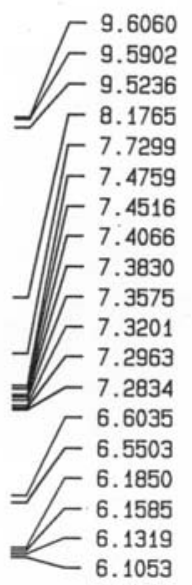

5.0963

F 5.0922

] 4.9561

$\Gamma^{4.1095}$

- 3.9351

- 3.8924

च 3.8505

- 3.8257

2.0369

1.8635

- 1.8417

F 1.8185

- 1.7954

- 1.7716

$\left[\begin{array}{r}1.7253 \\ -1.3304\end{array}\right.$

$-1.3304$

$-1.3205$

$-1.3090$

- 1.3032

$-1.2976$

$-1.2780$

$-1.2429$

$-1.2319$

$-1.0531$

$-1.0284$

L 1.0038

L 0.9574

$\leftarrow 0.6428$

$-0.1581$ 


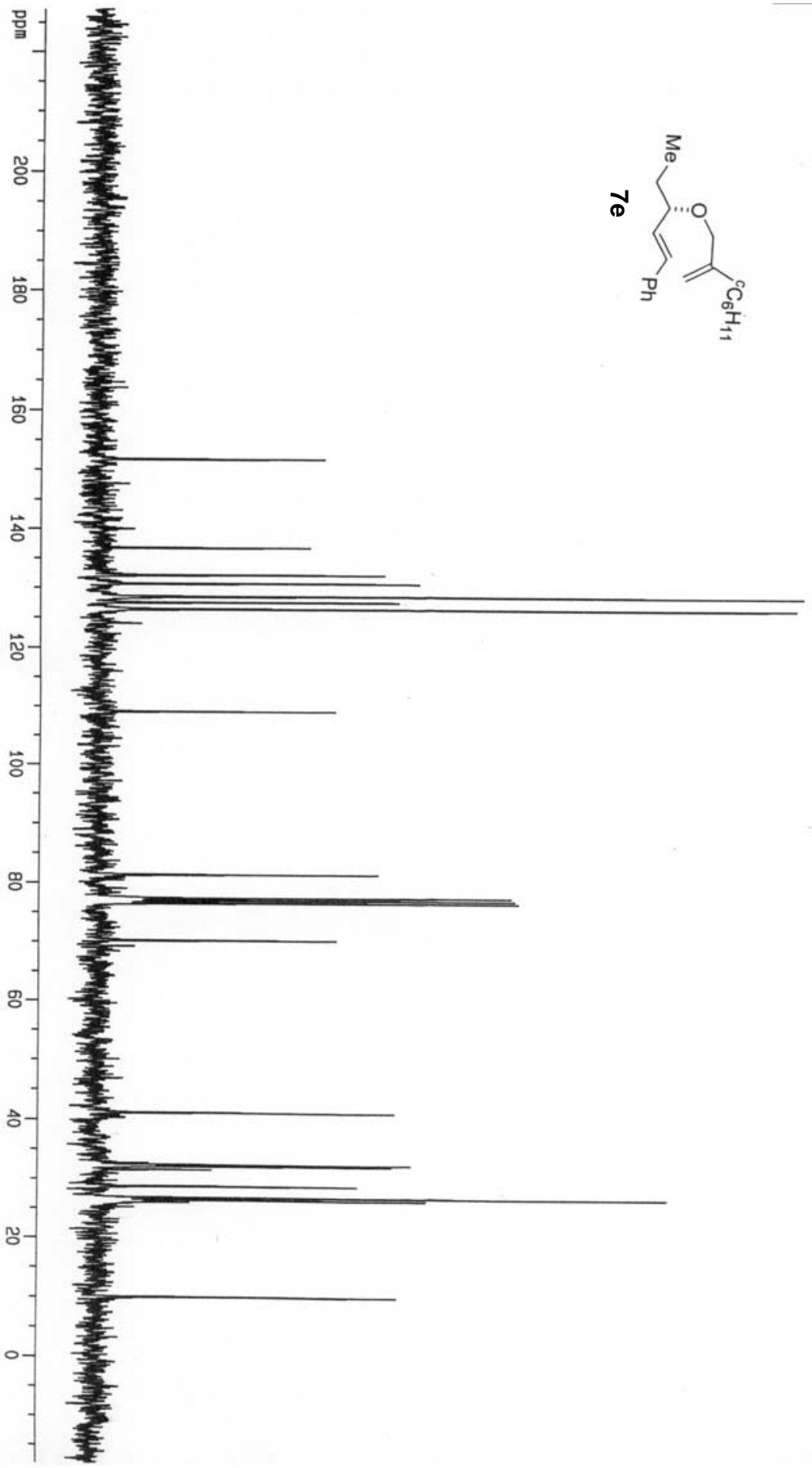

ppm
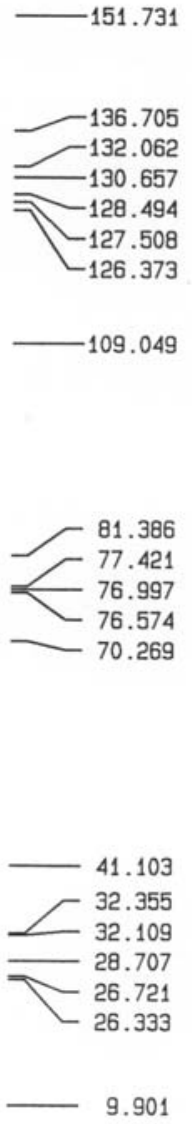


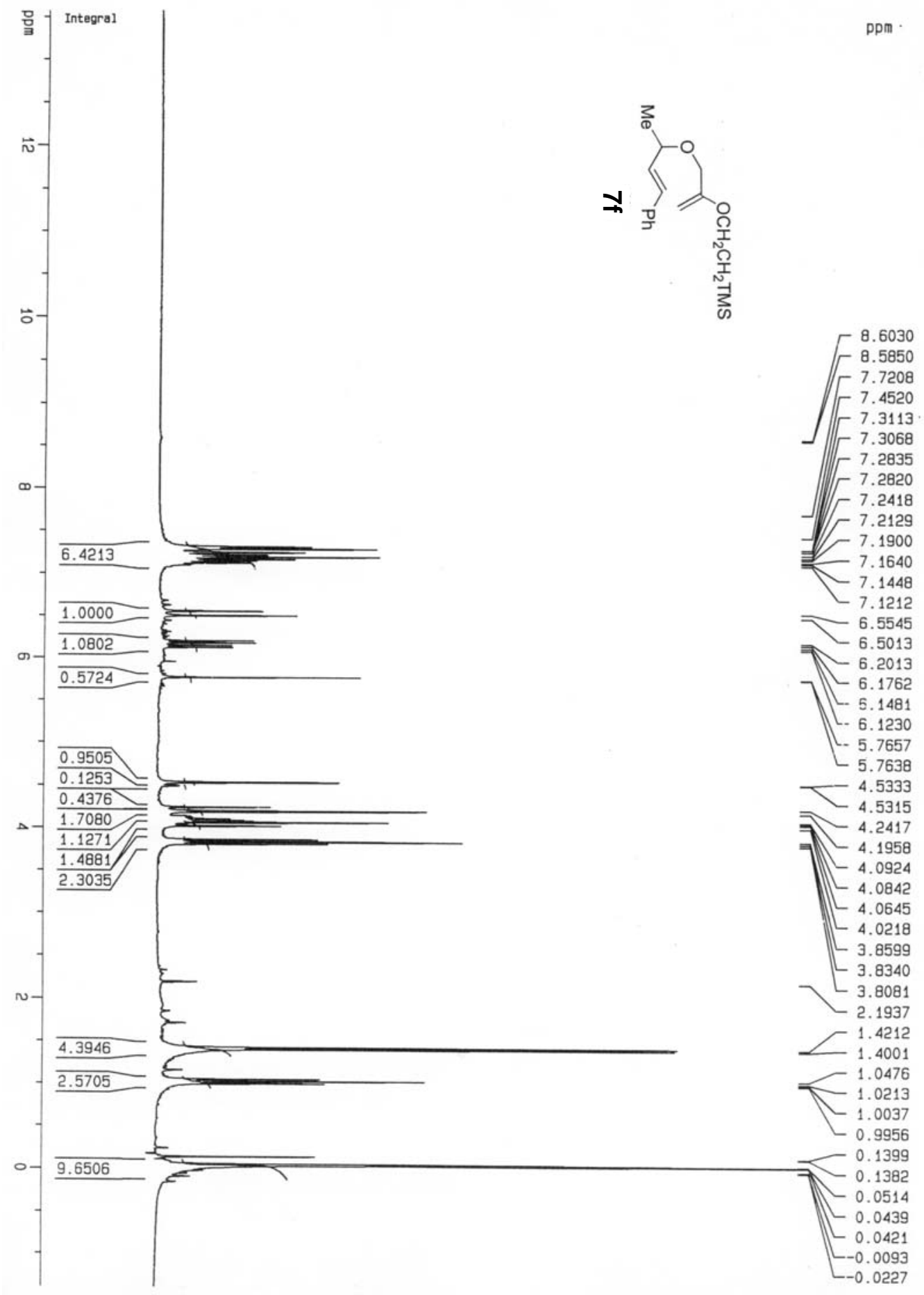




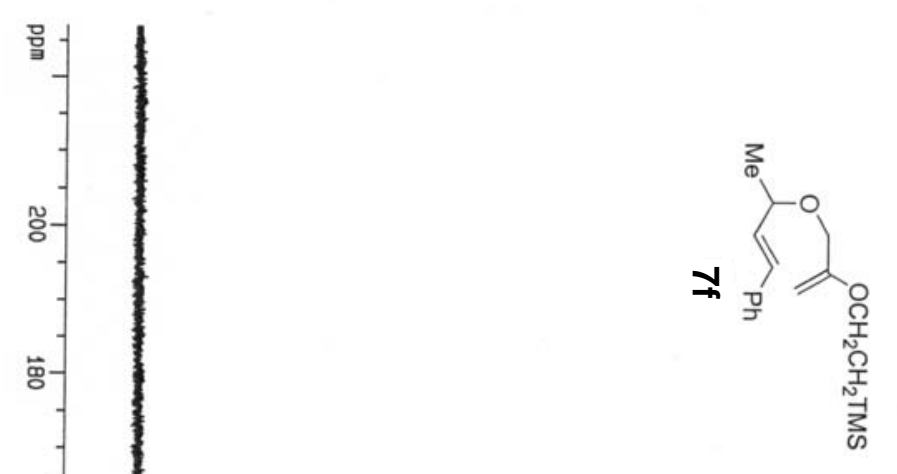

ppm

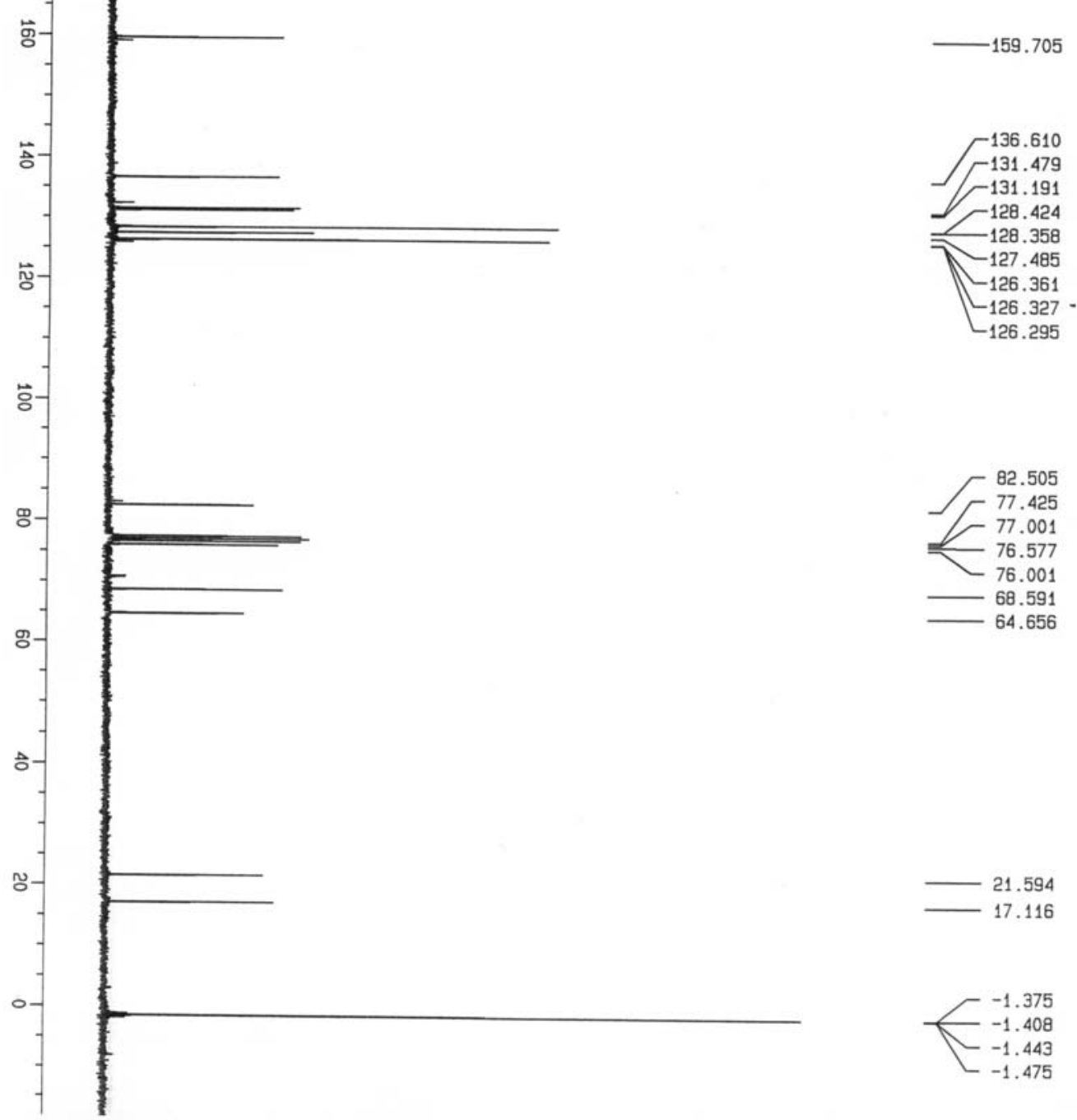




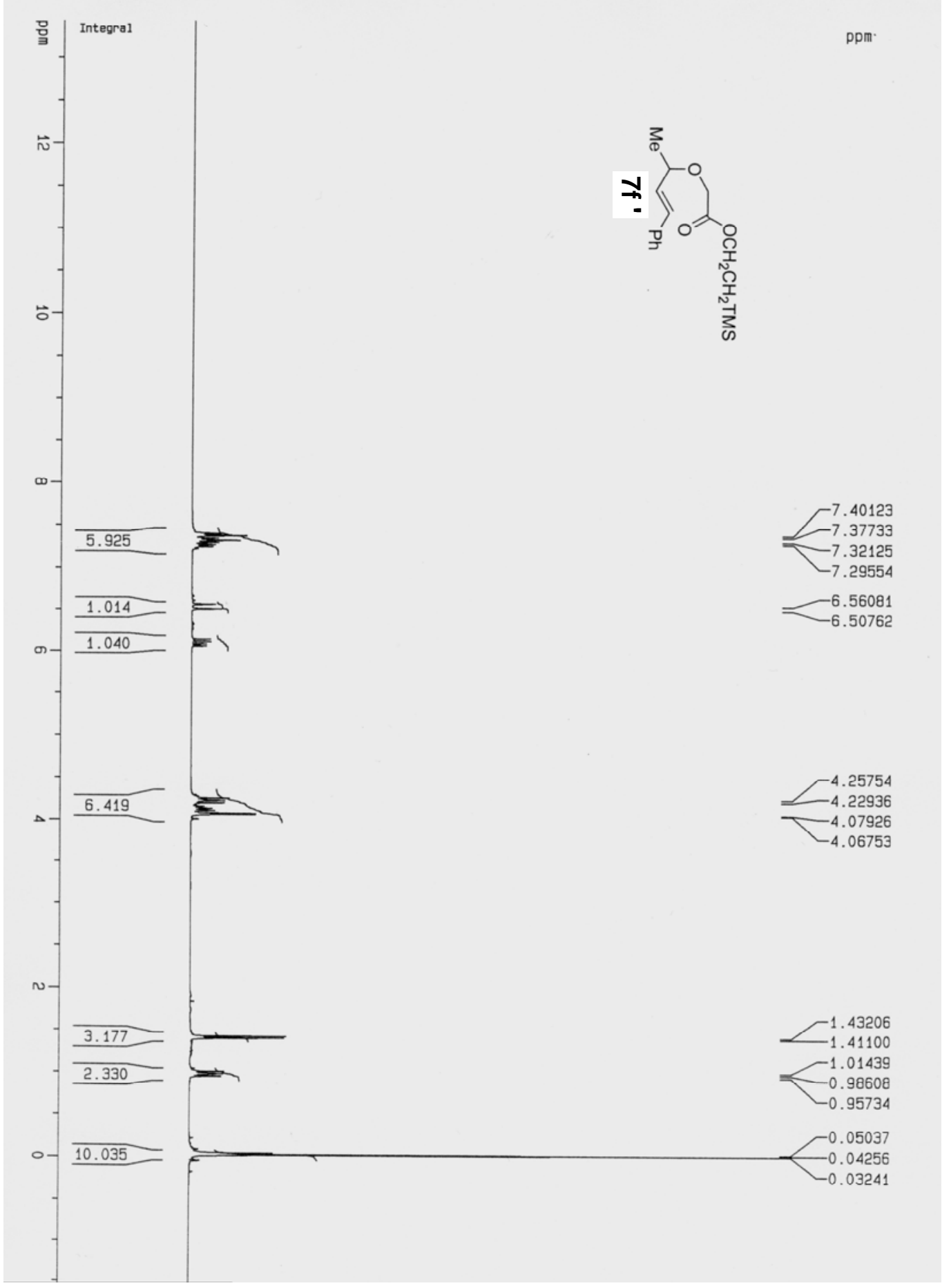




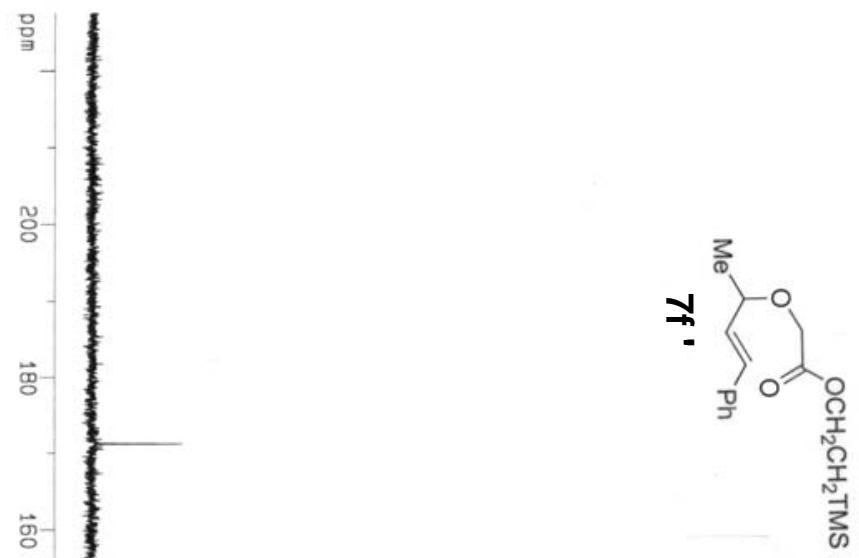

ppm
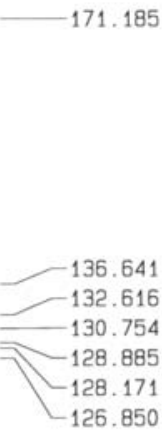

훙

曲
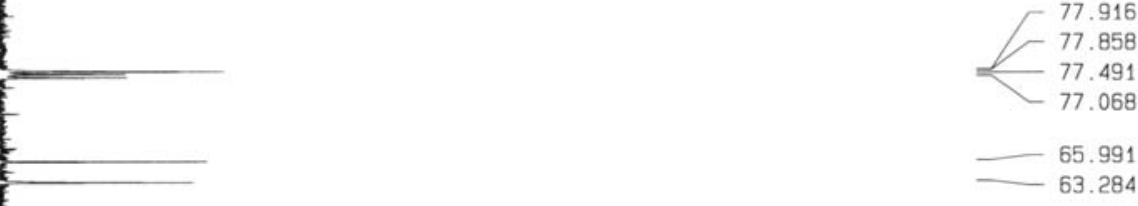

g-

ô

ก

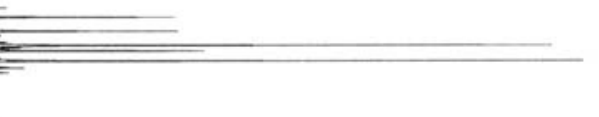
63.284

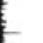




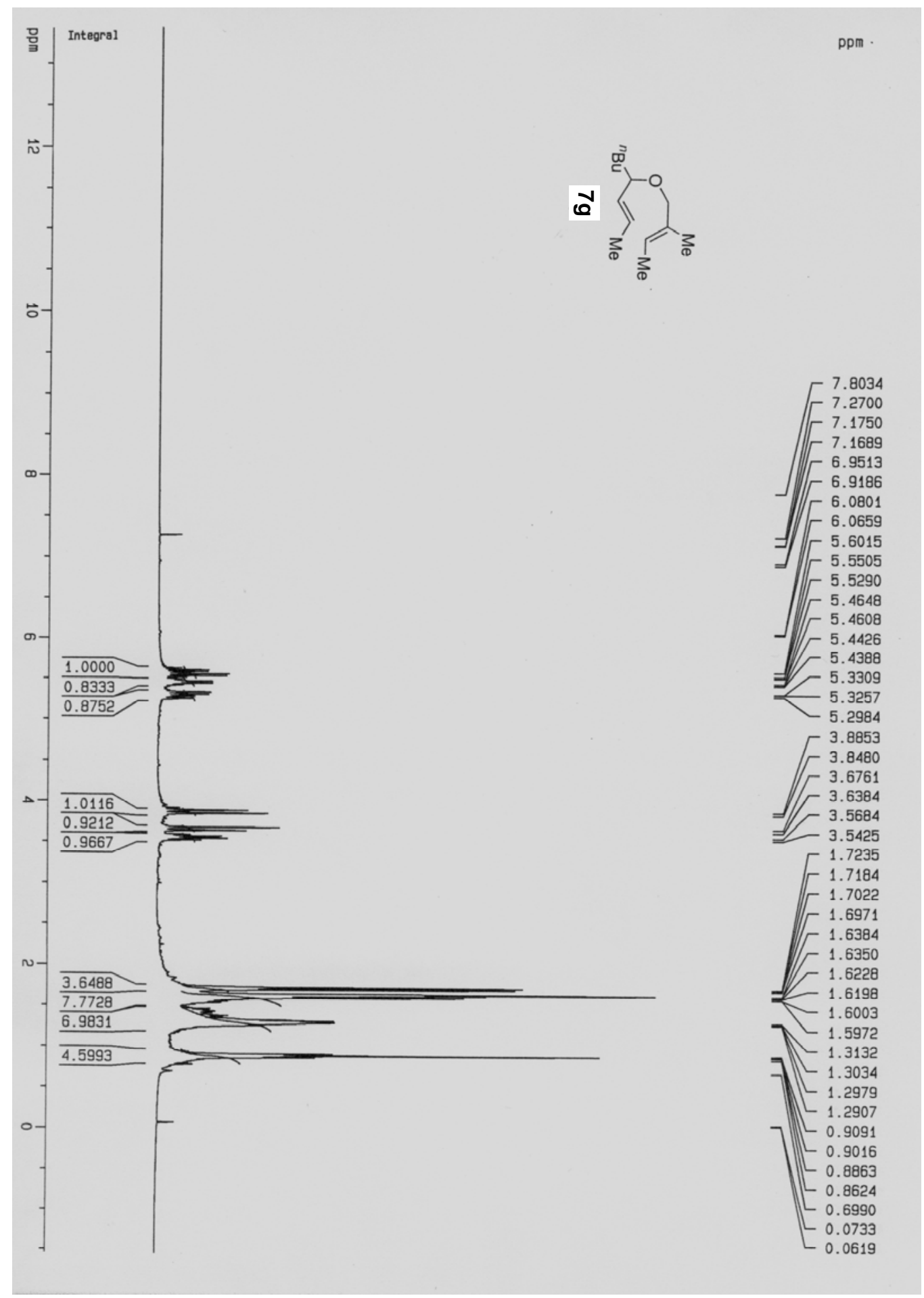




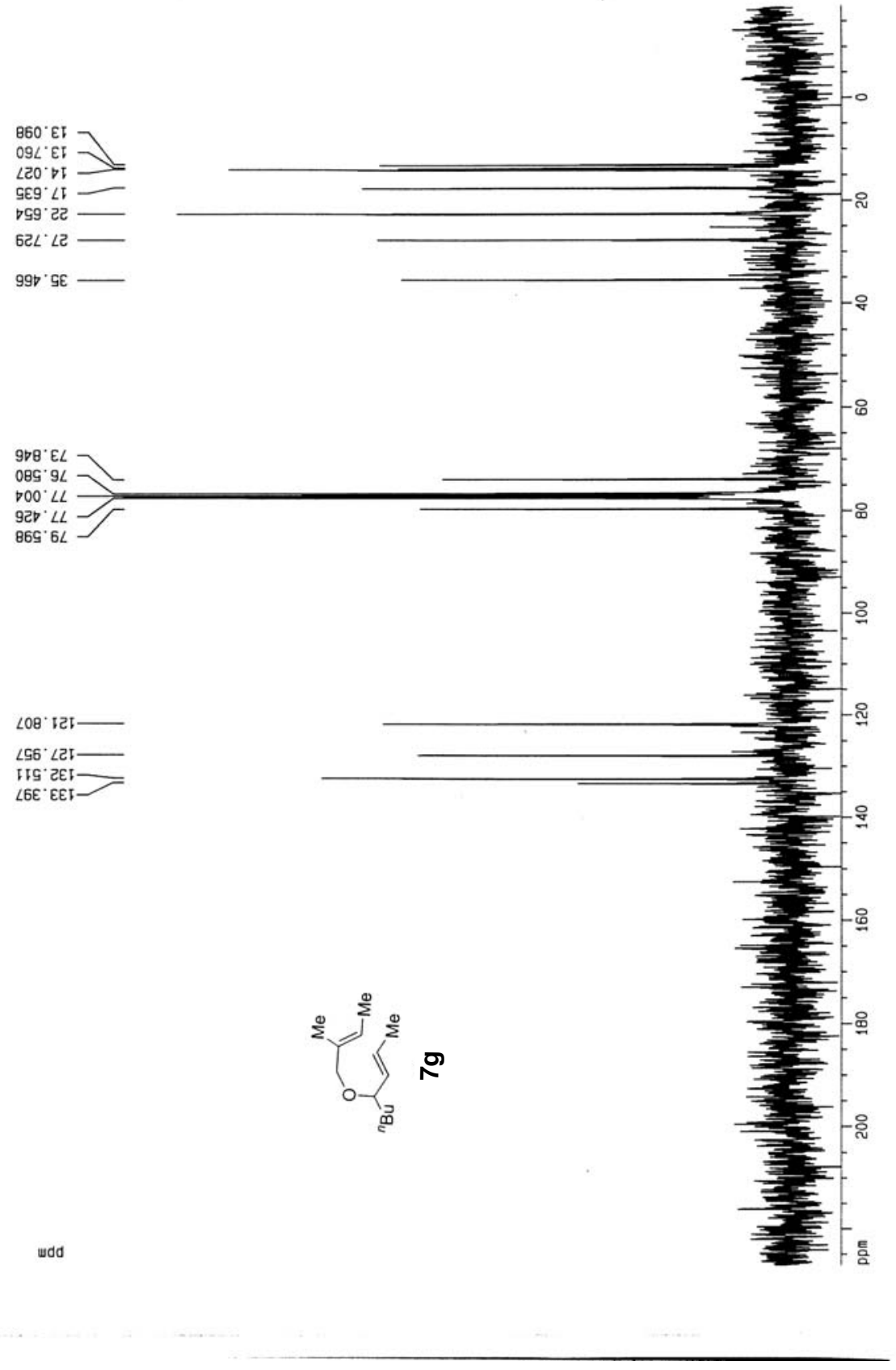




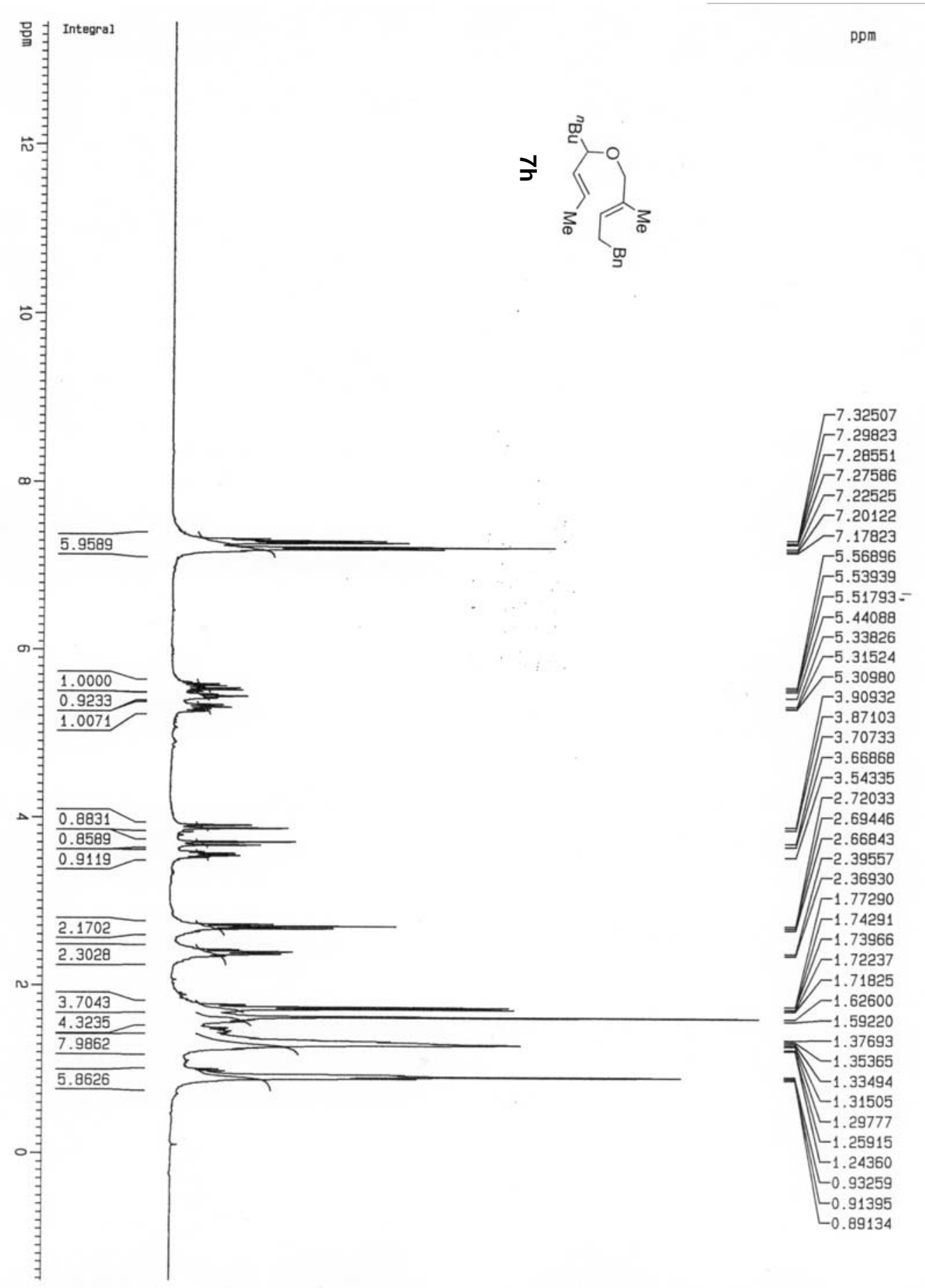




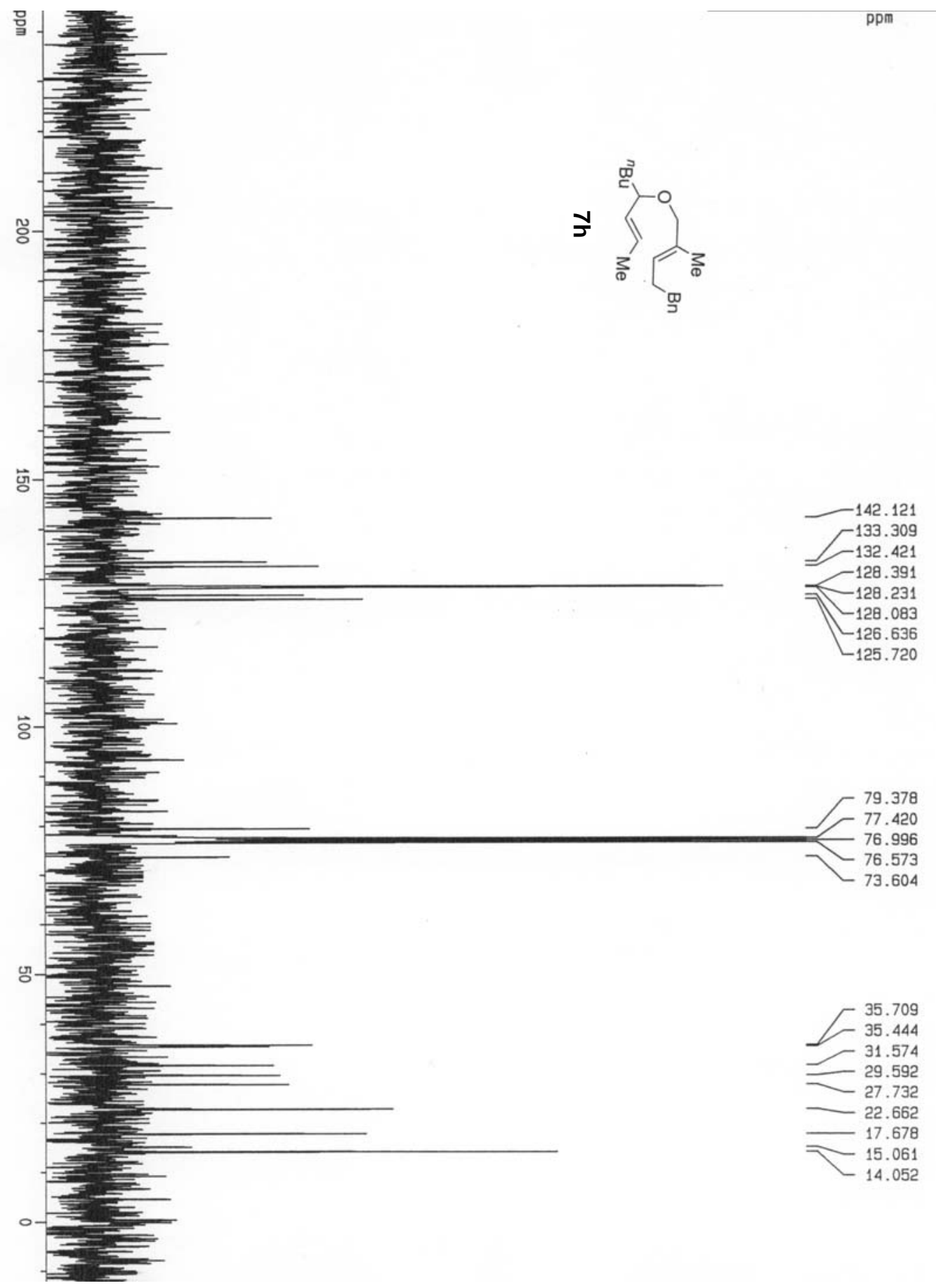



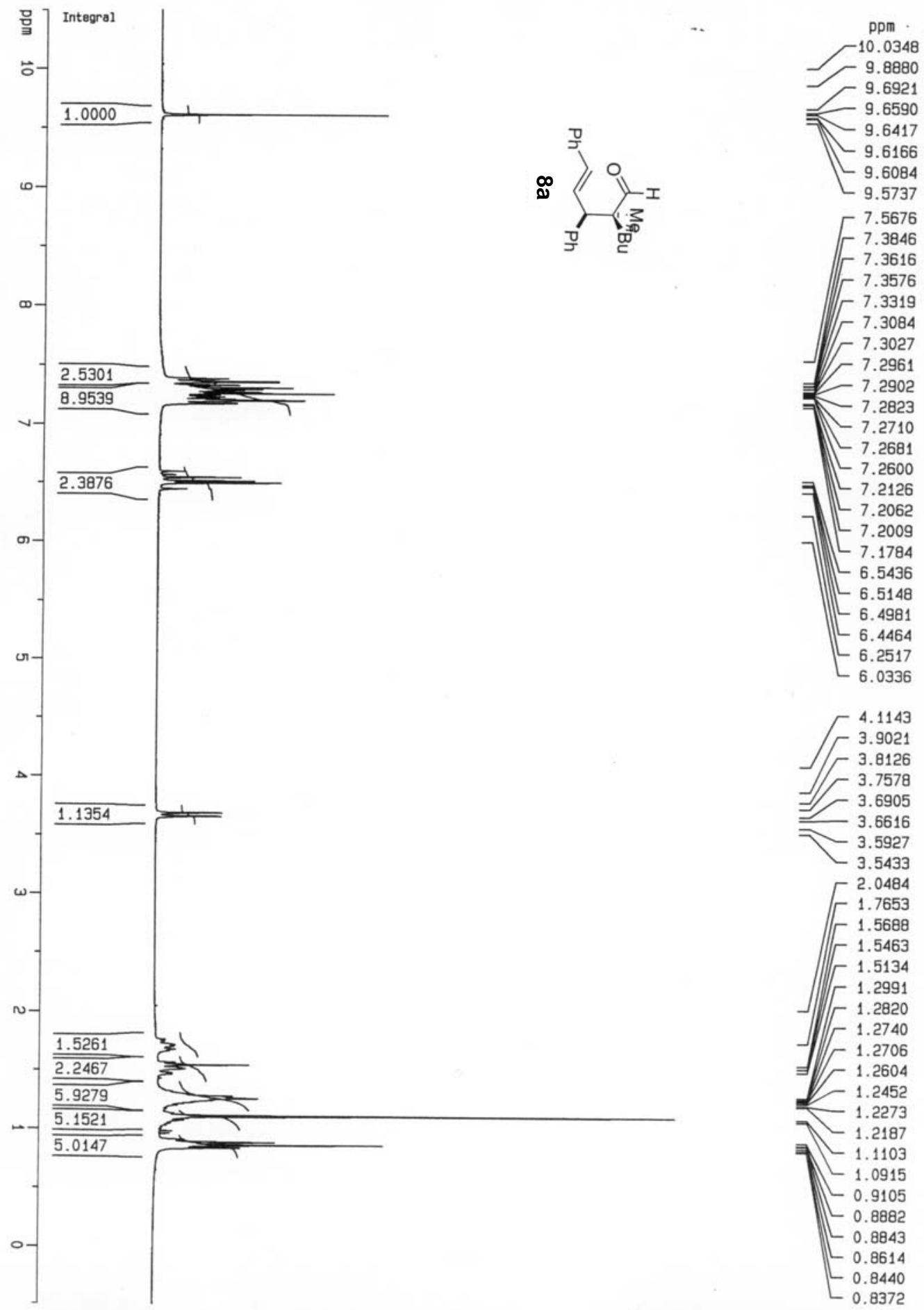


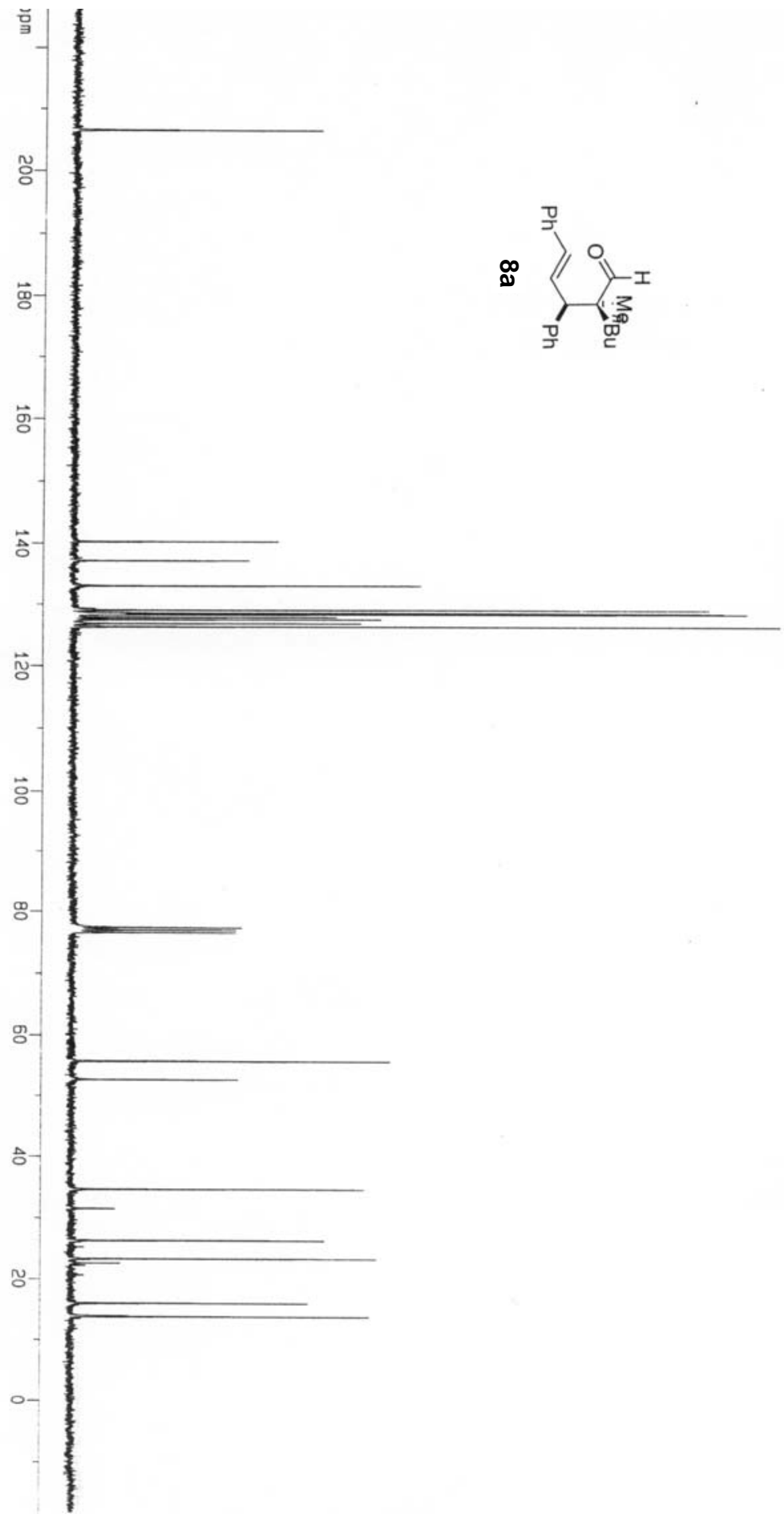

ppm

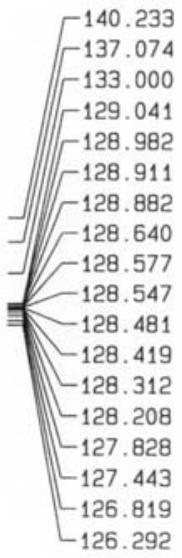
52.667 34.732 34.634

$-31.520$

- 26.310

- 25.253

$-23.318$

23.219

$-22.587$

- $\longleftarrow 22.263$

]$\leftarrow 20.659$

$-16.040$

$-14.037$

$-13.869$ 


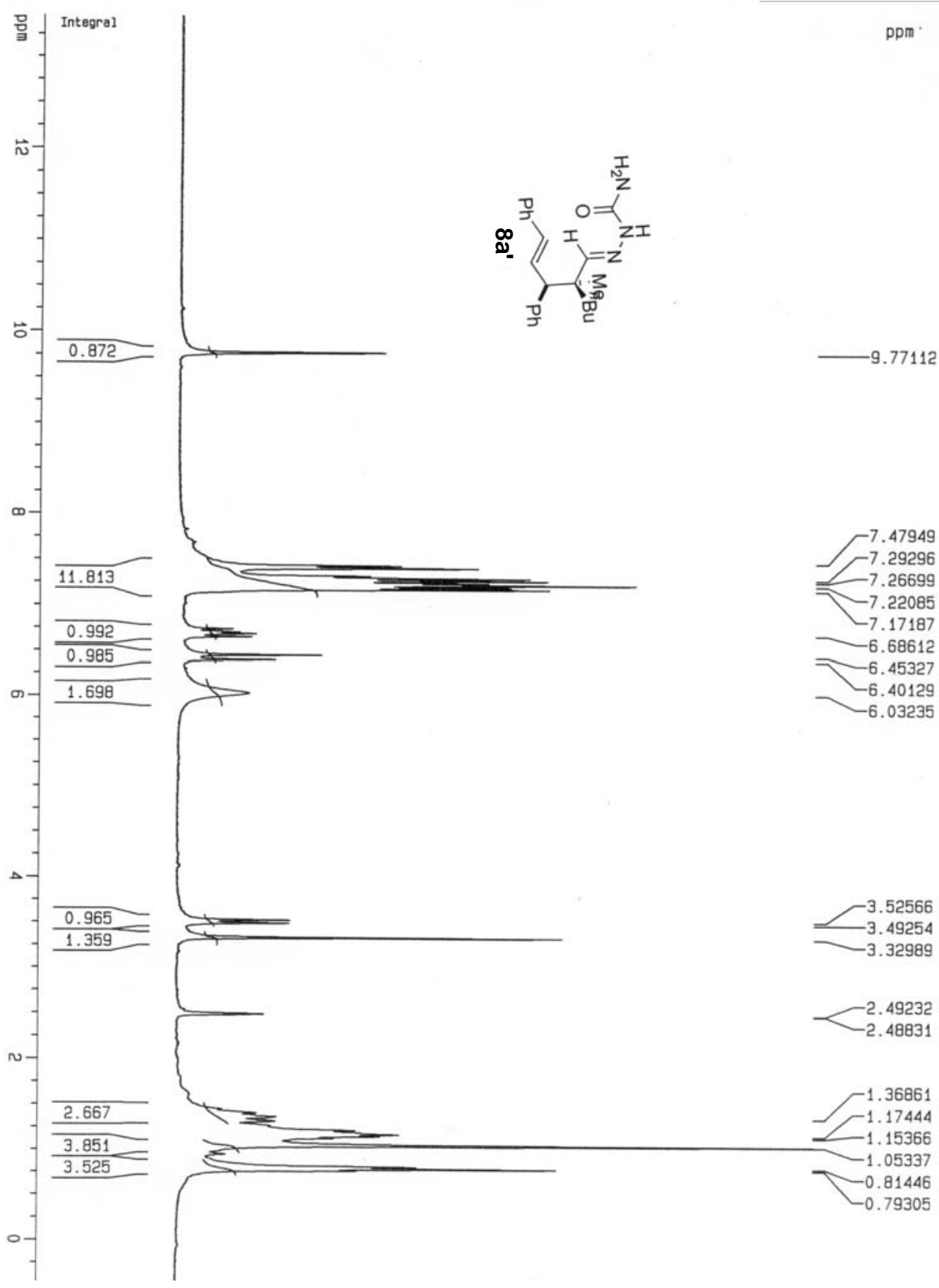




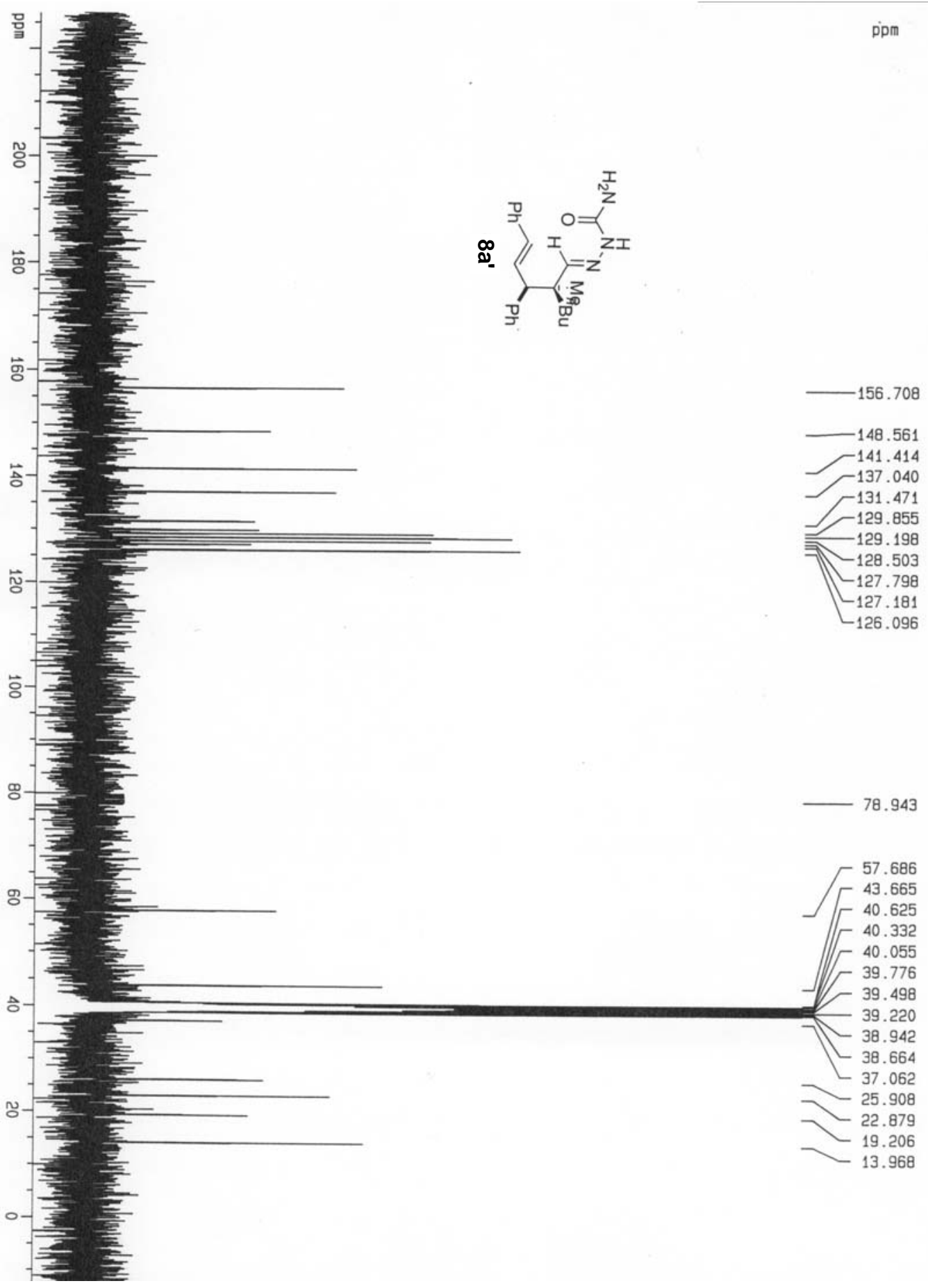



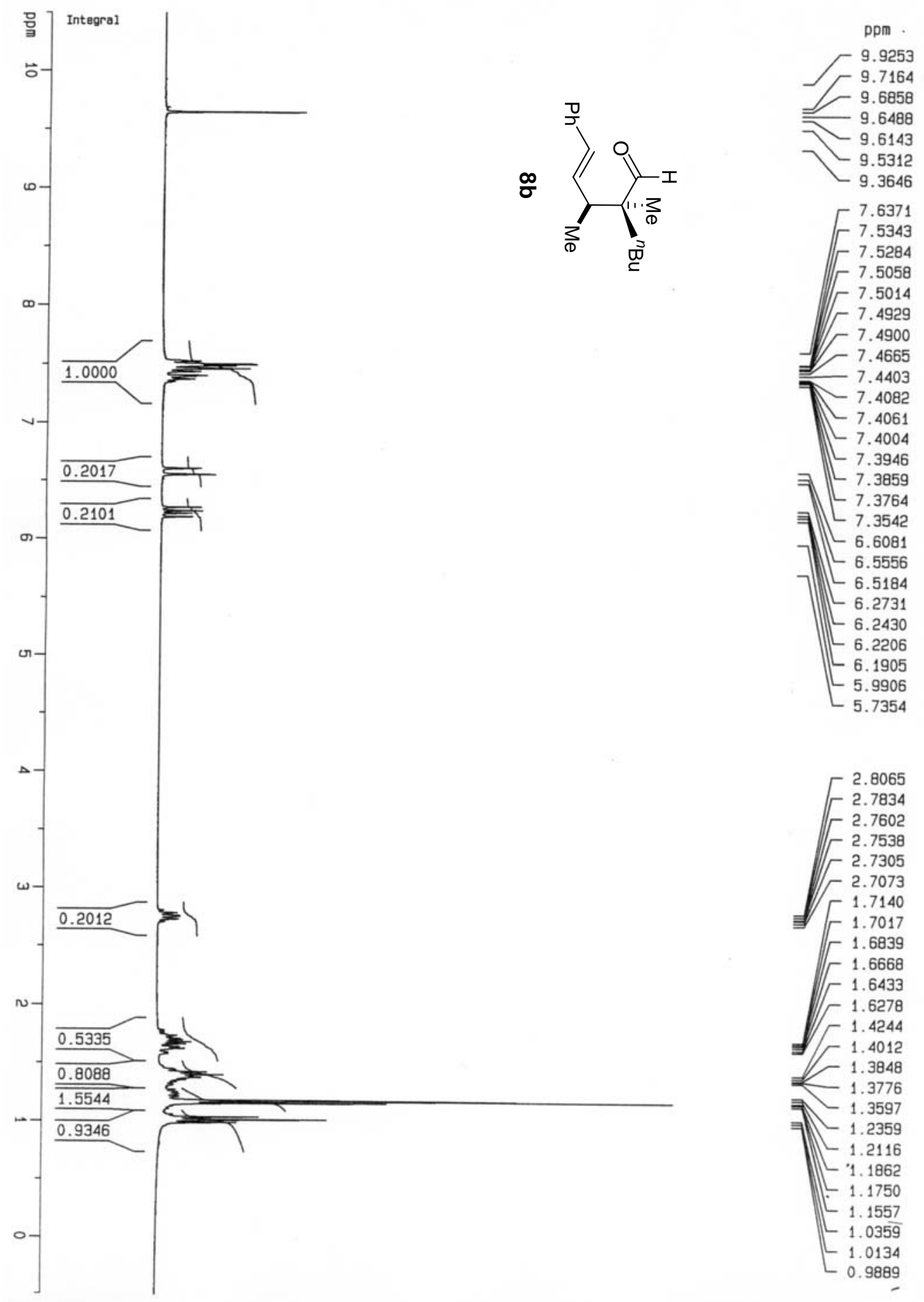


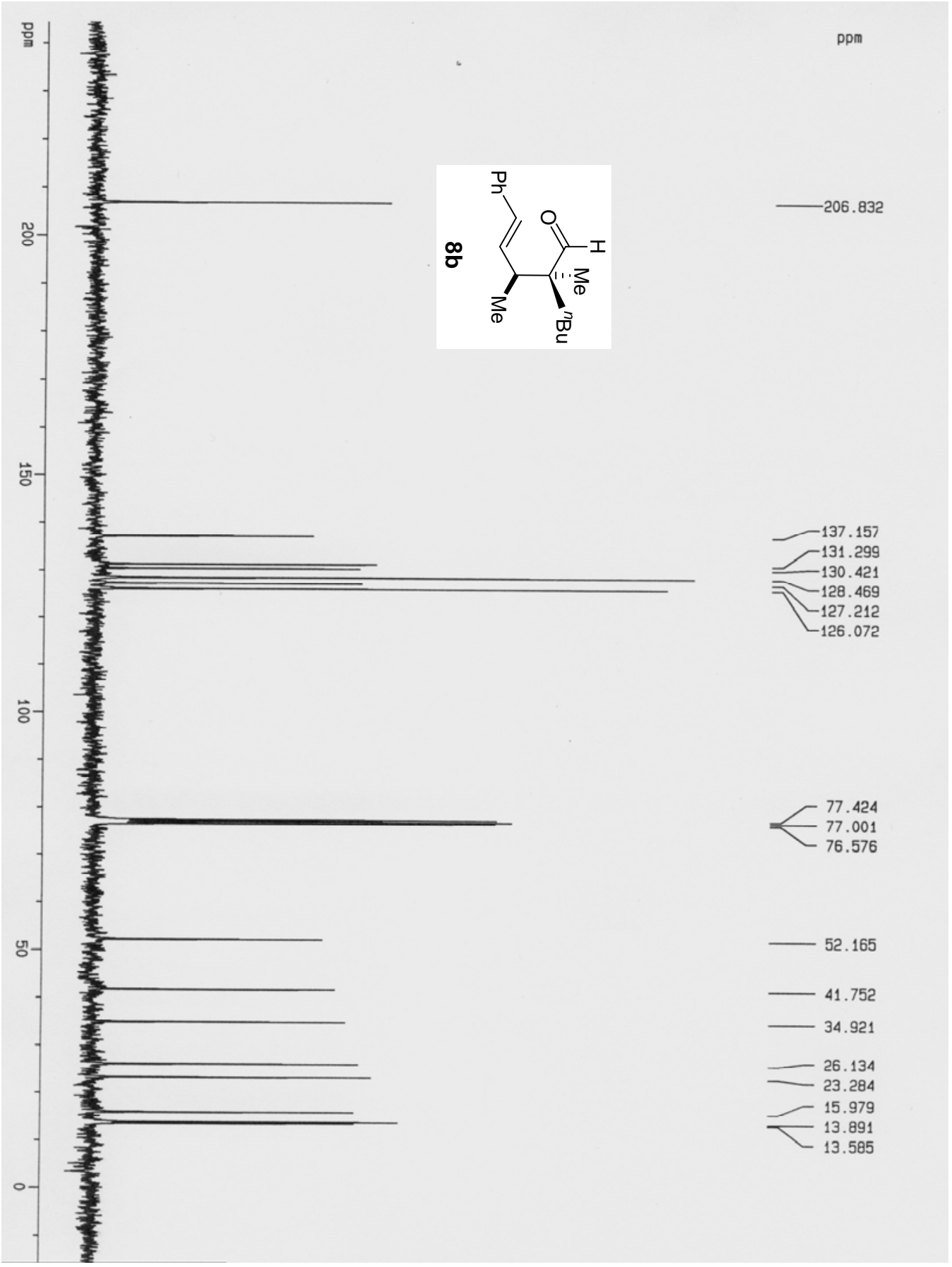




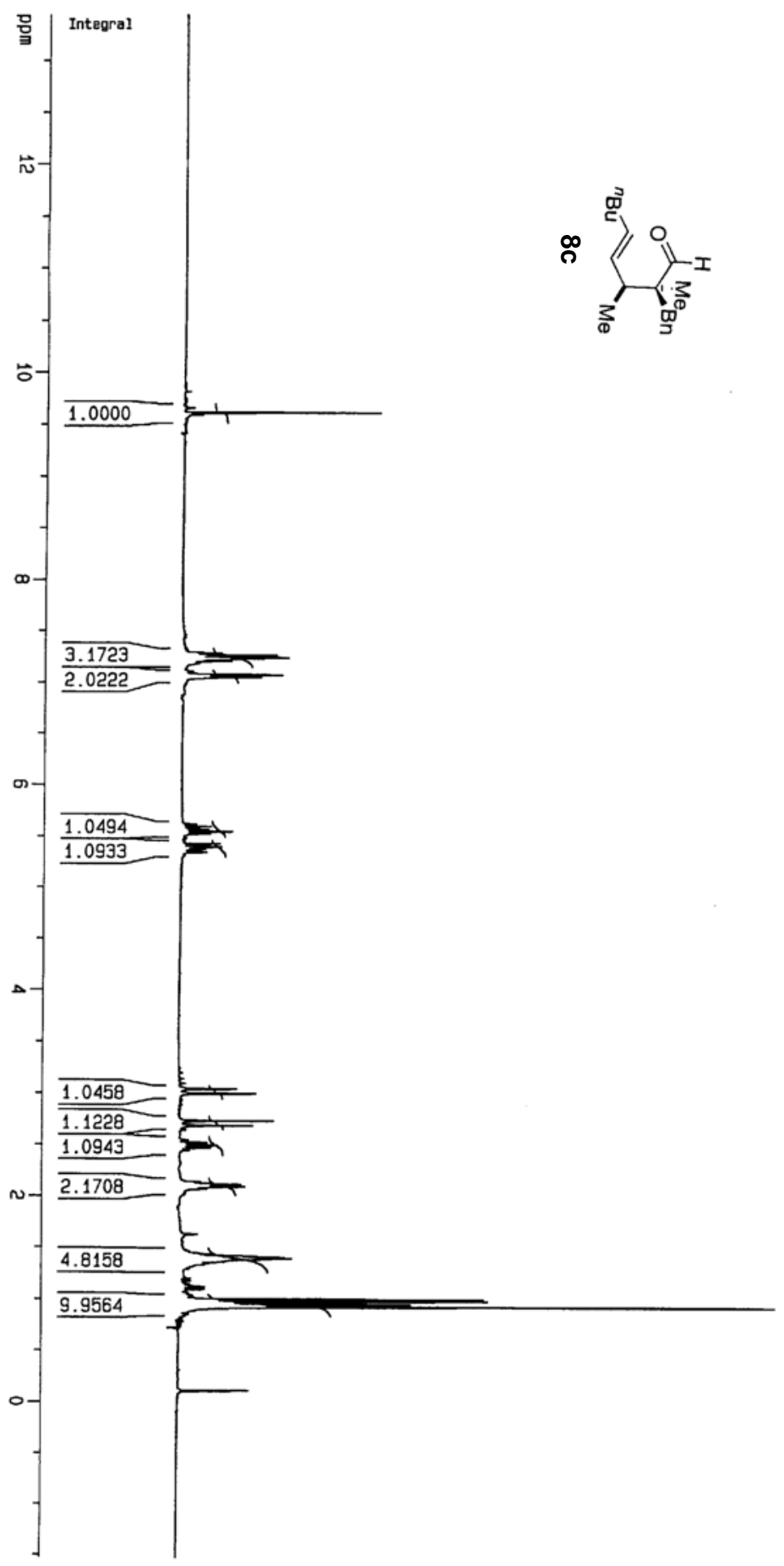

ppm

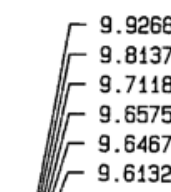

9.6132

9.5921
-9.5785
-9.5443

$-9.5443$

$-9.5239$

- 9.4160

7.2819

7.2796

7.2628

7.2587

7.2443

- 7.2392

$\begin{array}{r}7.2371 \\ -7.2278 \\ \hline\end{array}$

$7-7.2225$

$-7.2046$

$-7.0782$

L 7.0723

L 7.0513

7.0470

$-5.5411$

-5.4224
-5.3929

- 5.3888

F $\begin{array}{r}3.0377 \\ 2.9917\end{array}$

2.7273

2.6814

- 2. 4949

- 2.4898

T.1111

2.0887

$=1.4208$

$\sqrt{1.4149}$

F 1.4102

F 1.3011

$-1.3810$

$-1.3720$

$7-1.3654$

$\mathbb{L}_{1.3596}$

$-1.0011$

L 0.9780

L 0.9492

40.9412

L 0.9252

0.1222

$-0.1100$ 


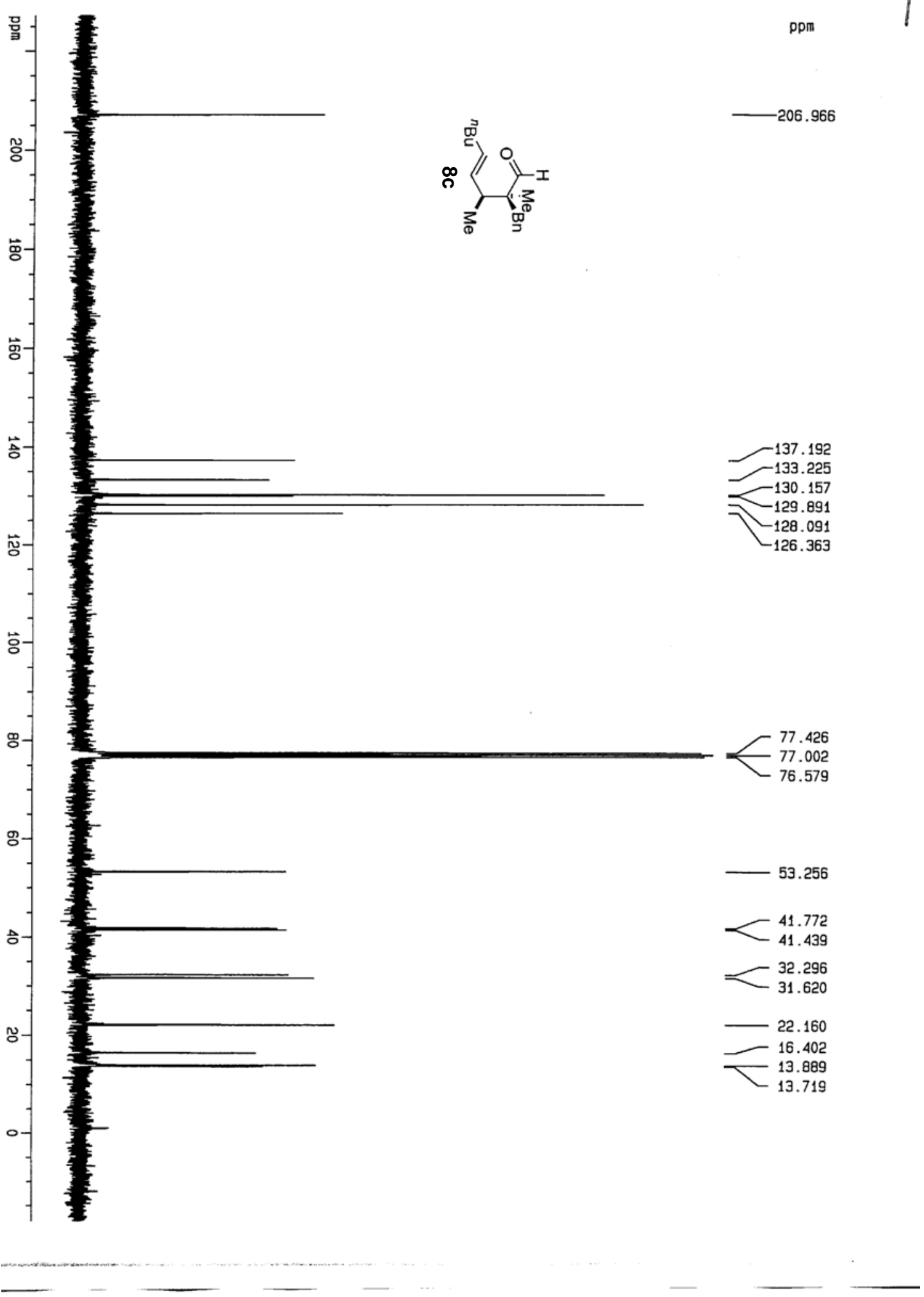




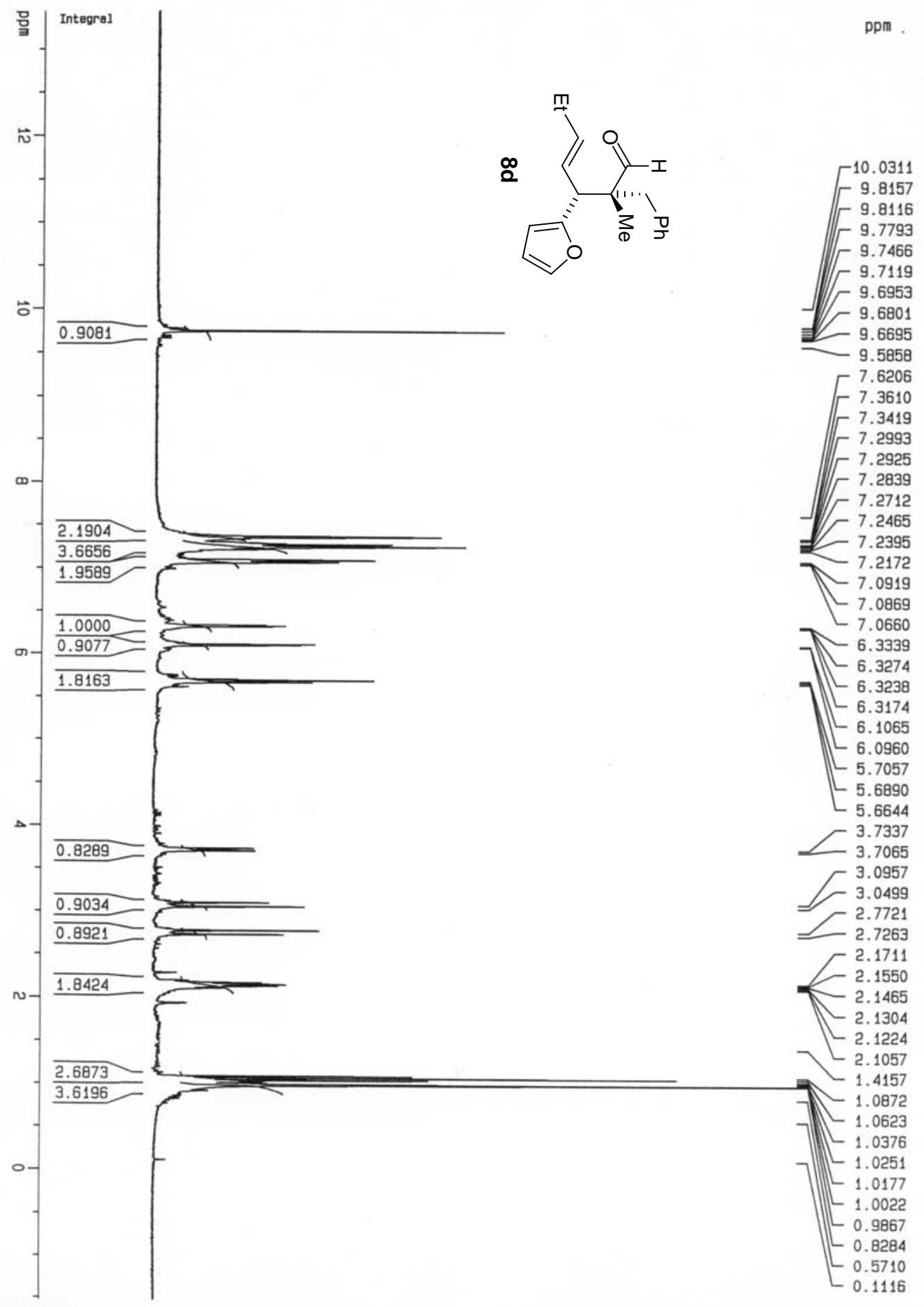




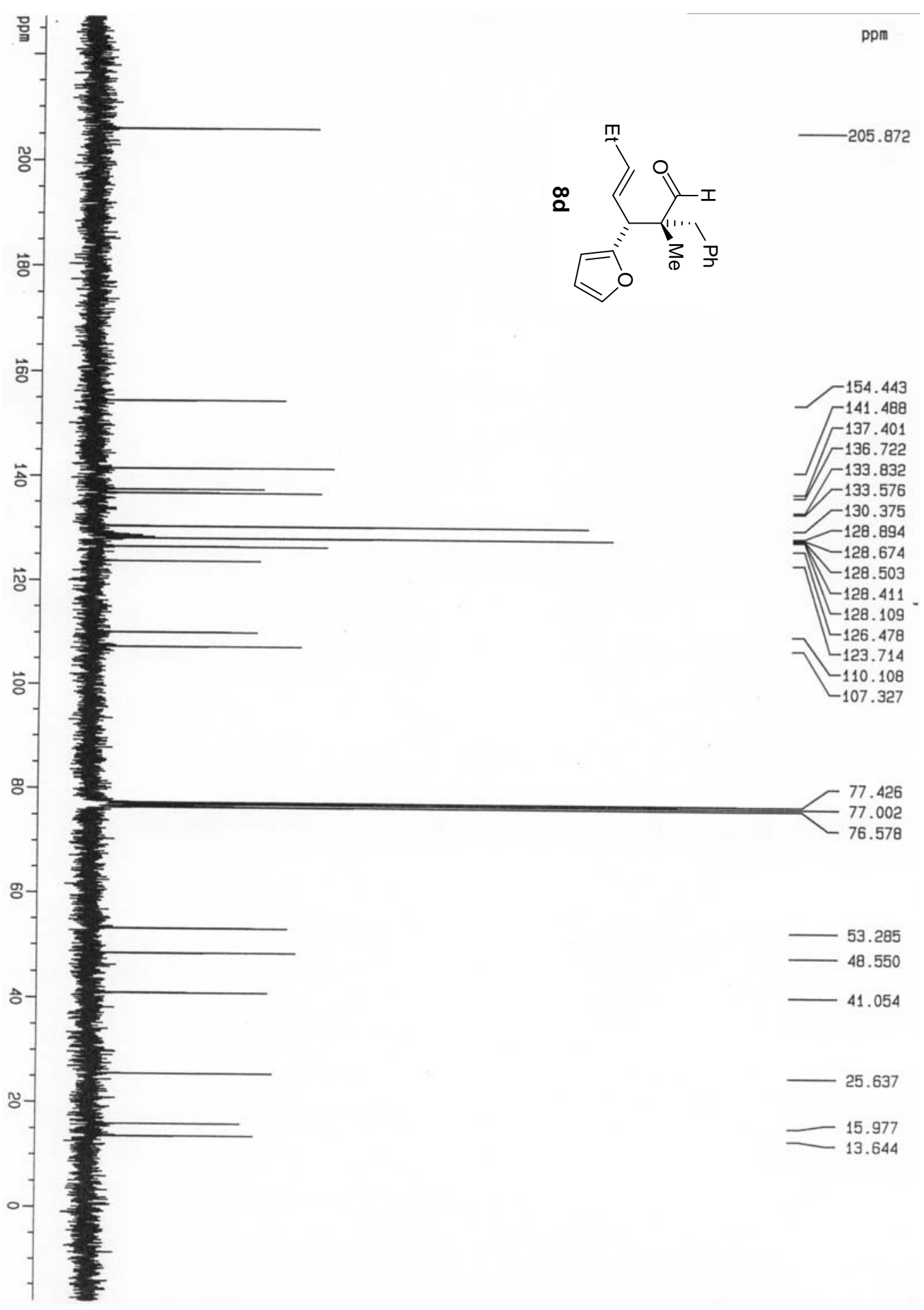




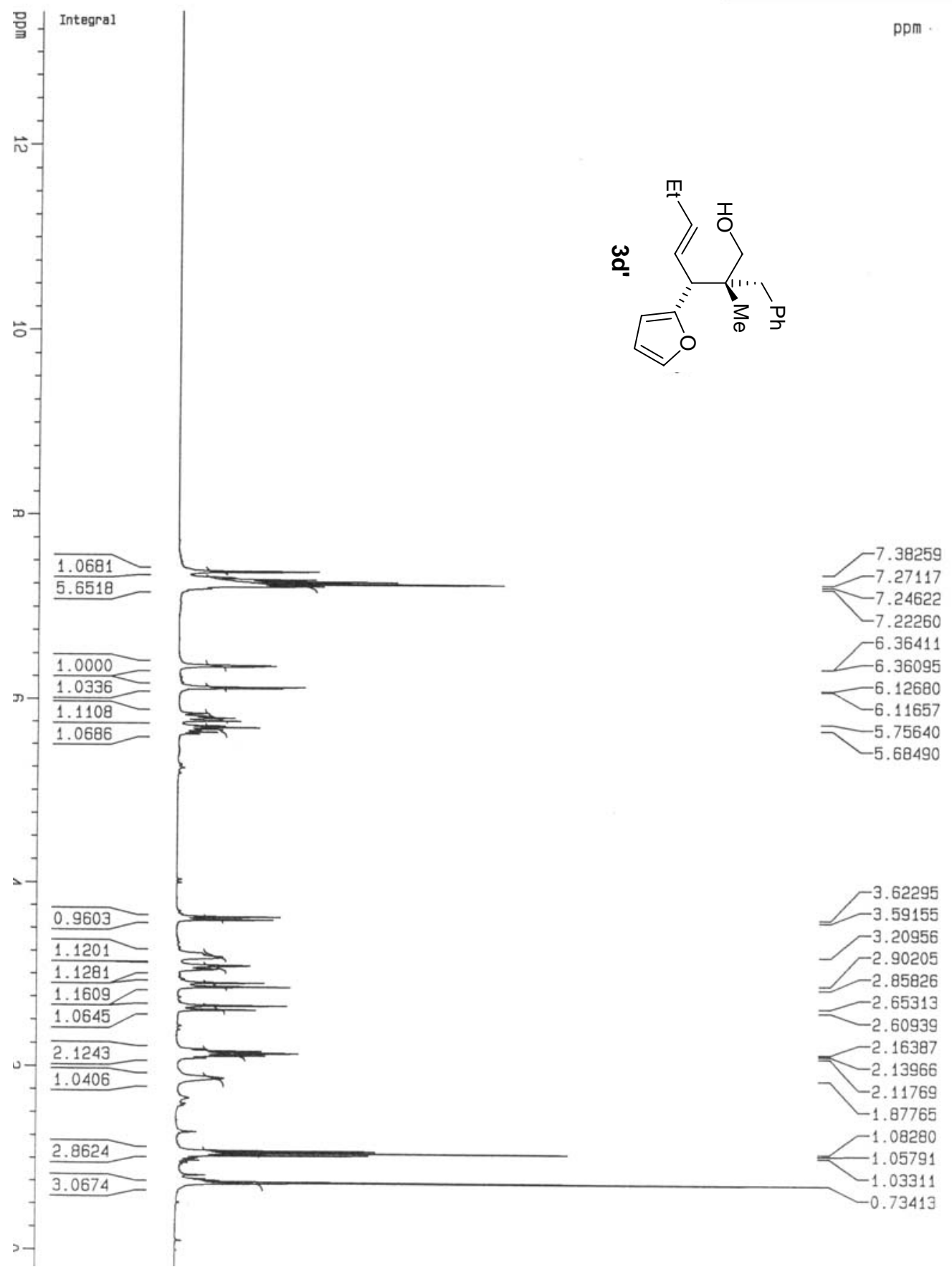




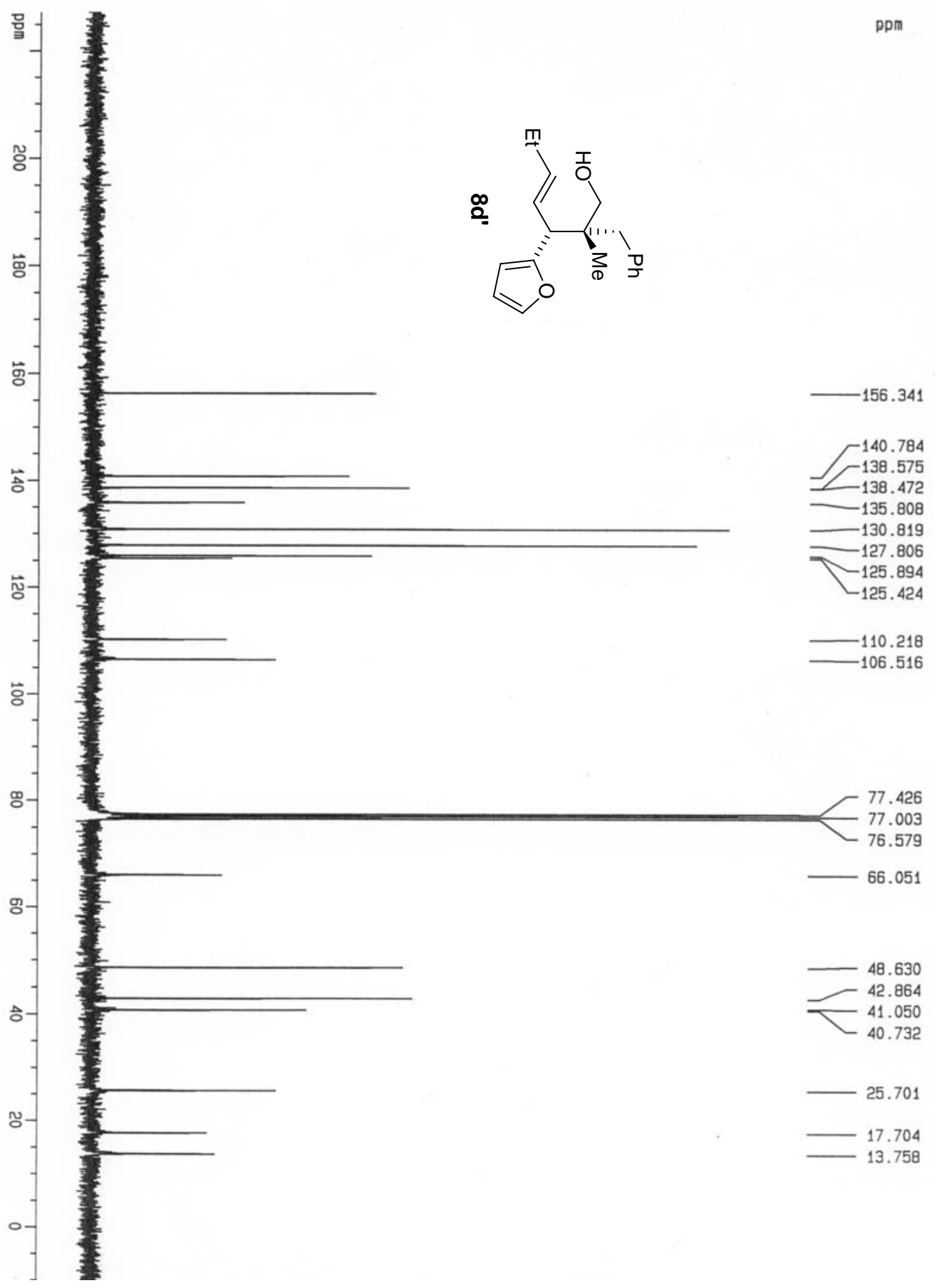




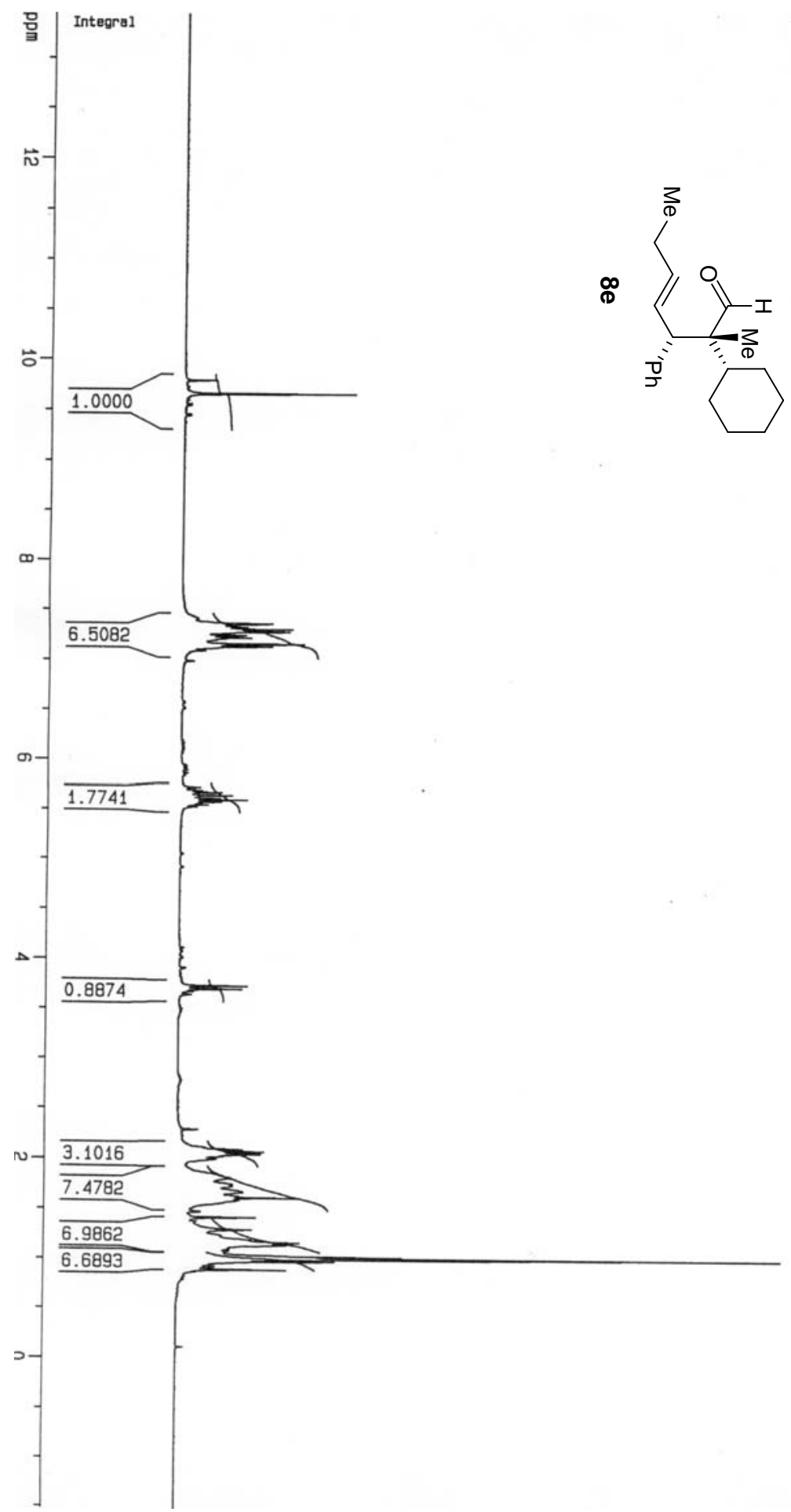

$-9.79762$

$-9.73455$

$-9.66805$

9.60060

$-9.56452$

$-9.54940$

9.49709
$\quad 9.46097$

$-9.44754$

7.41952

$-7.36481$

-7.36200

$-7.33177$

-7.32650

$-7.30867$

$-7.30390$

$-7.29512$

$-7.28958$

$-7.24875$

$-7.24443$

$-7.22475$

$-7.16583$

$-7.16096$

$-7.13791$

$-5.67091$

$-5.63925$

$-5.61338$

$-5.59419$

$-5.57392$

$\begin{array}{r}3.72957 \\ -3.69823 \\ \hline\end{array}$

2.08816

$-2.06479$

$-2.04143$

1.67241

${ }^{-1.66509}$

$-1.61002$

$-1.59090$

$-1.41514$

$-1.29178$

$-1.18824$

$-1.16138$

$-1.13724$

$-1.03152$

$-1.02227$

$-1.00874$

L 0.99765

$-0.98248$

$-0.97271$

$-0.88889$

$-0.62074$

$\leftarrow_{0.10774}$ 


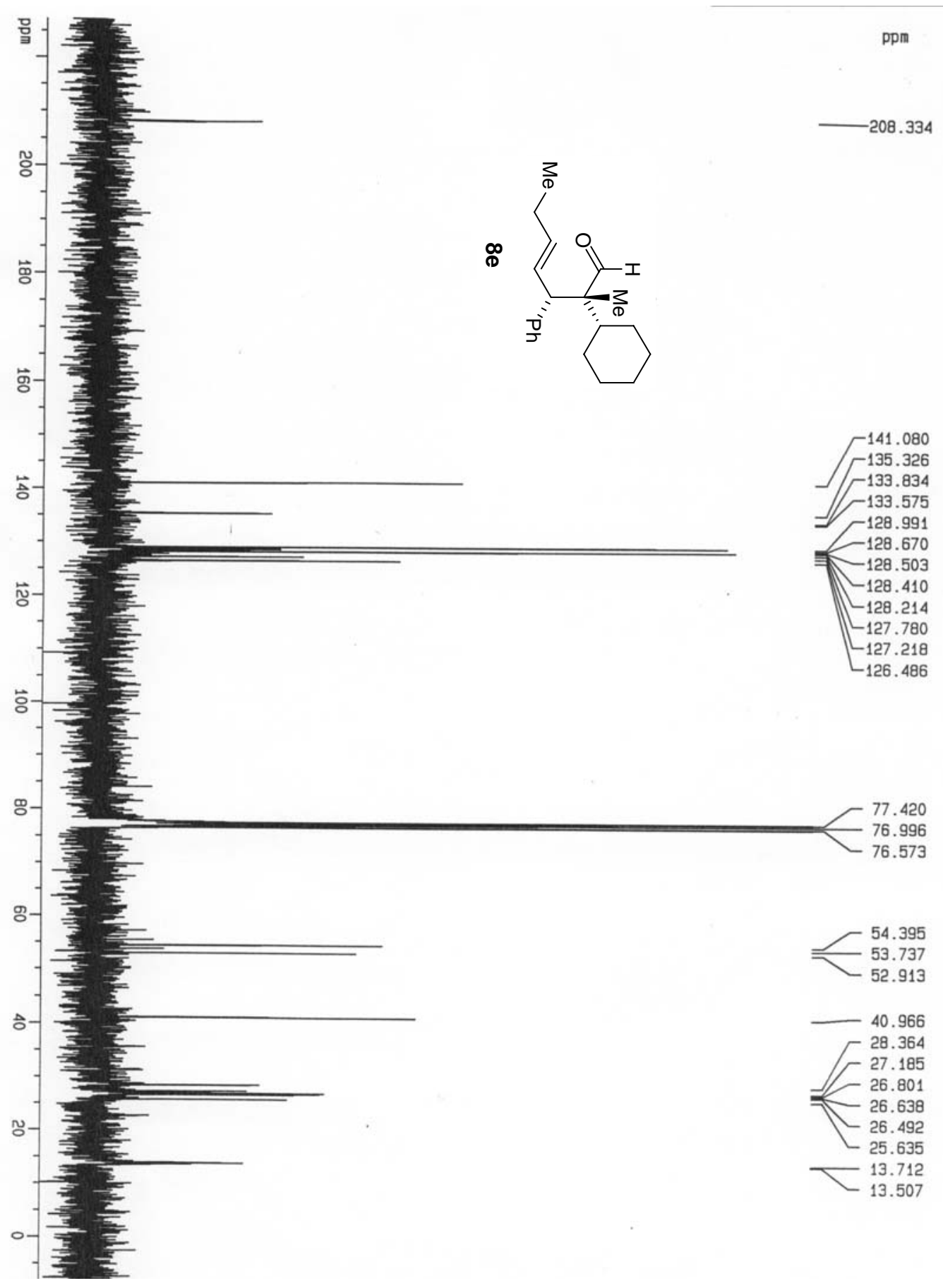




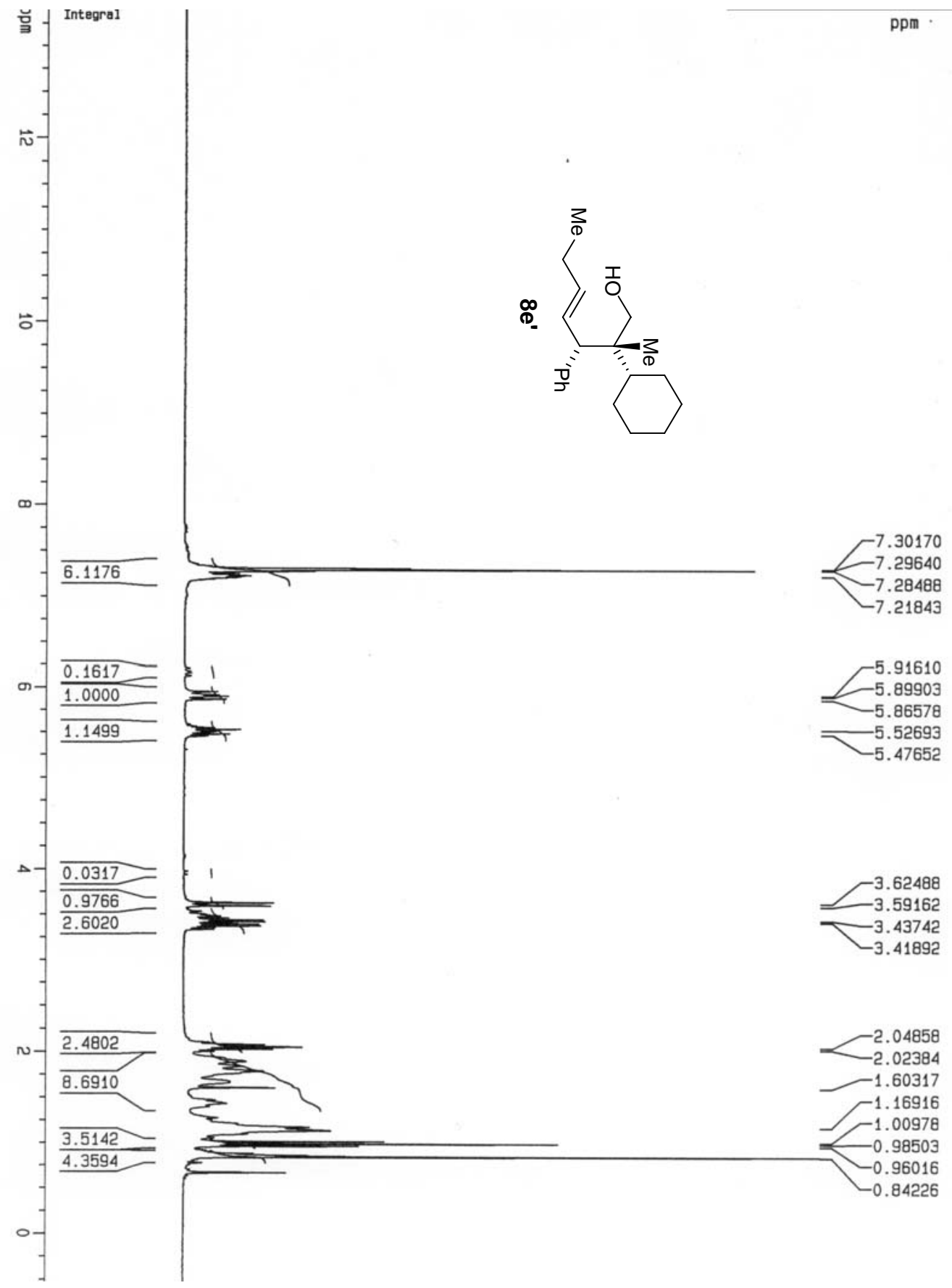




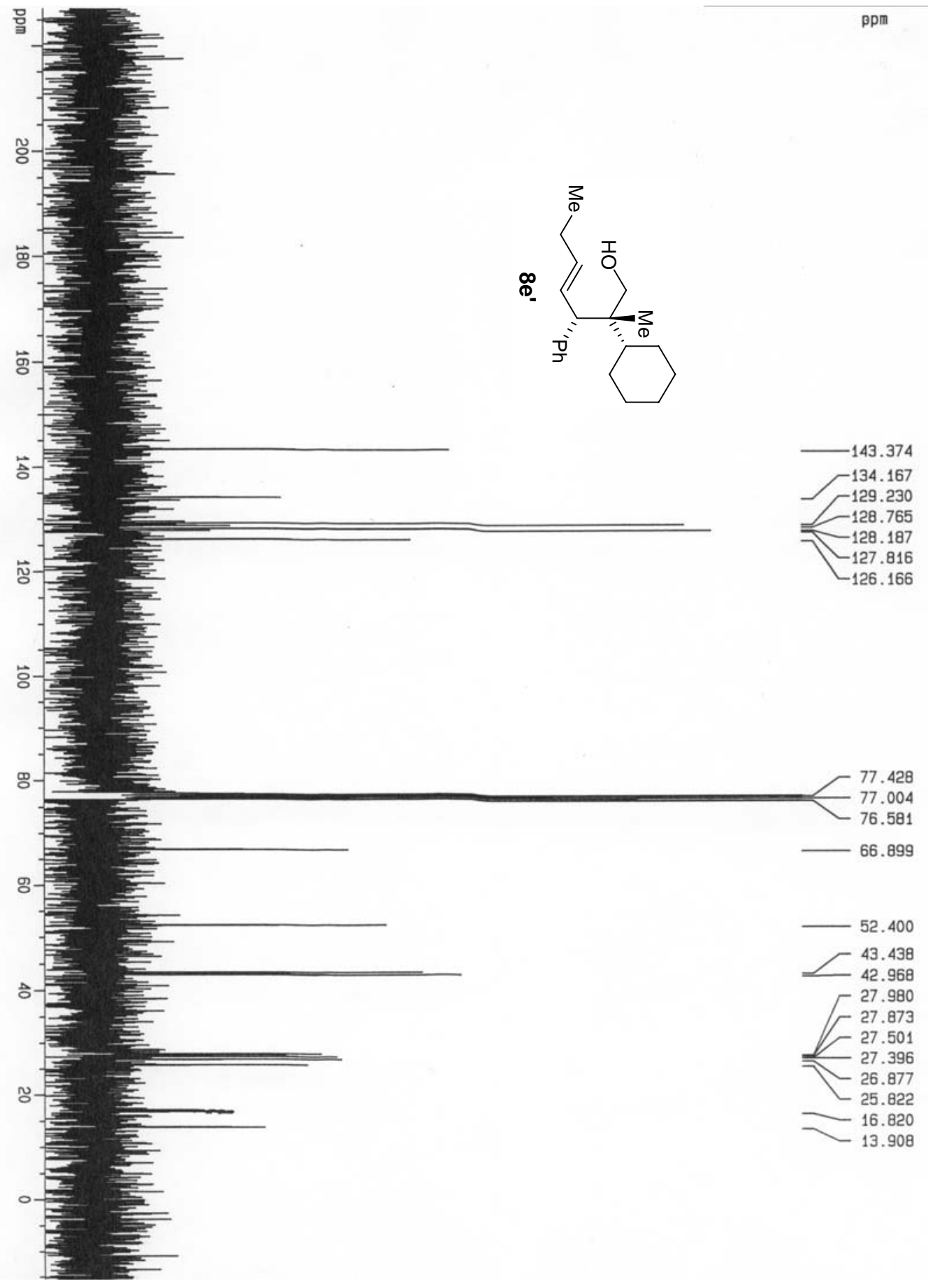




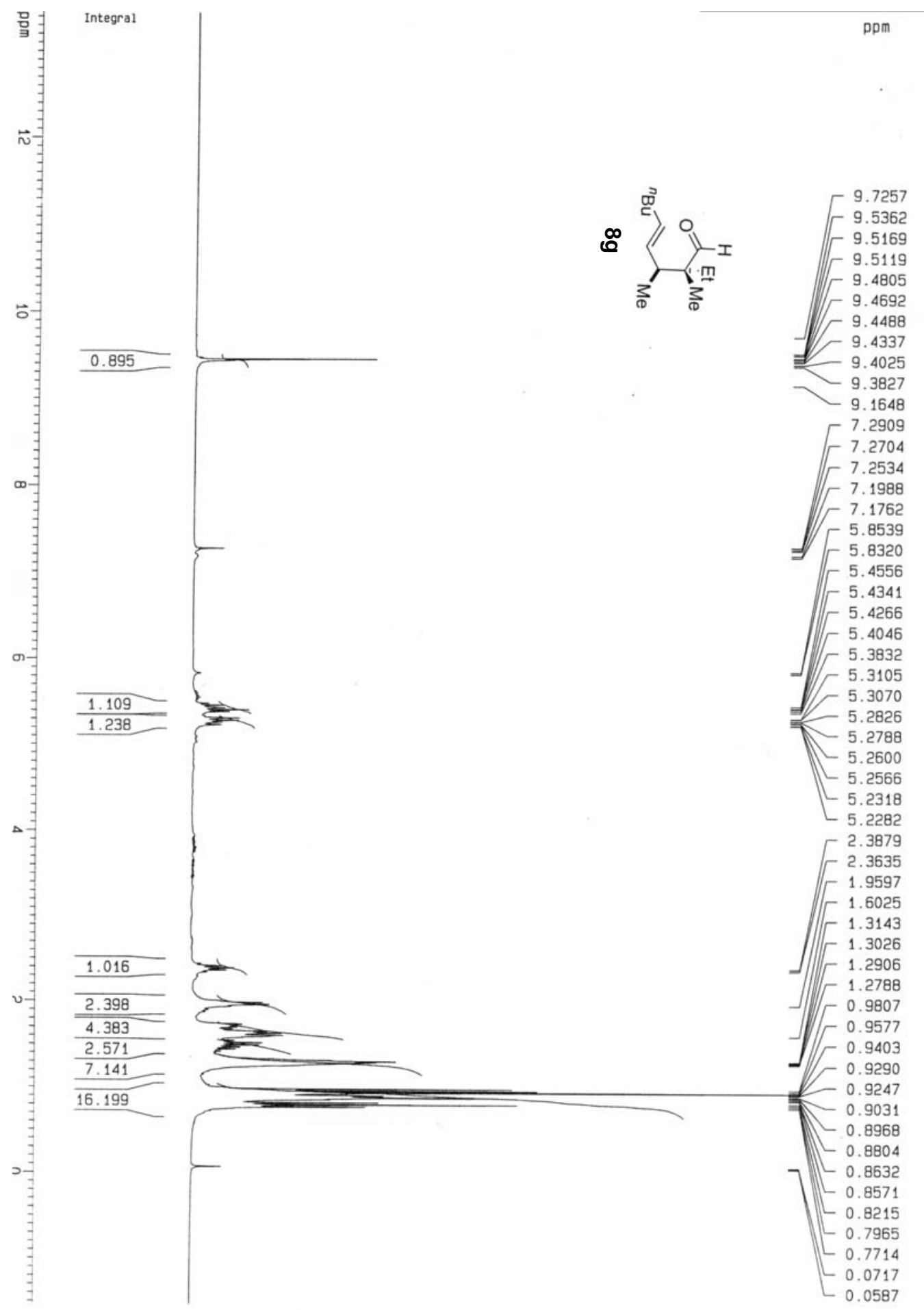



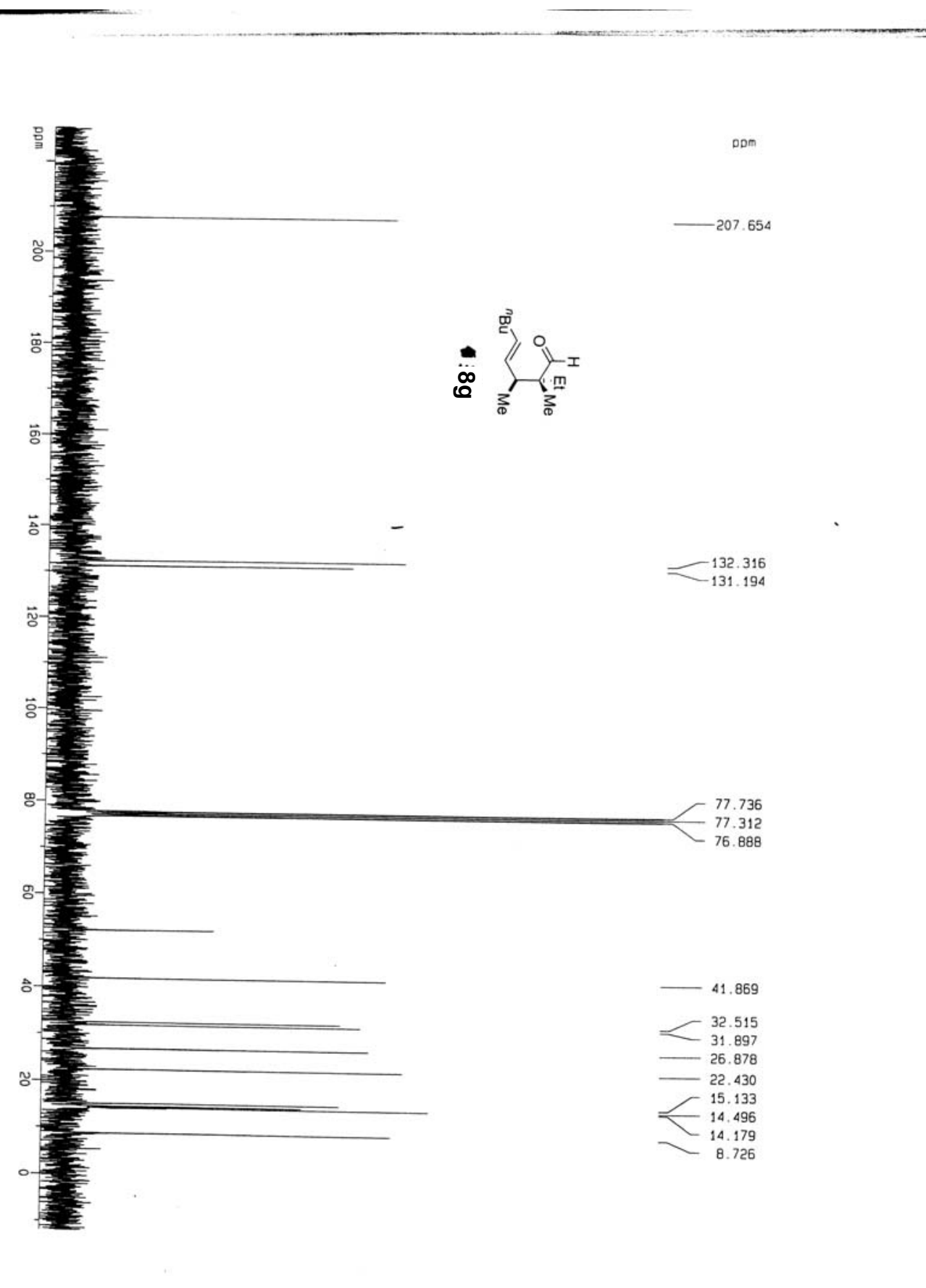


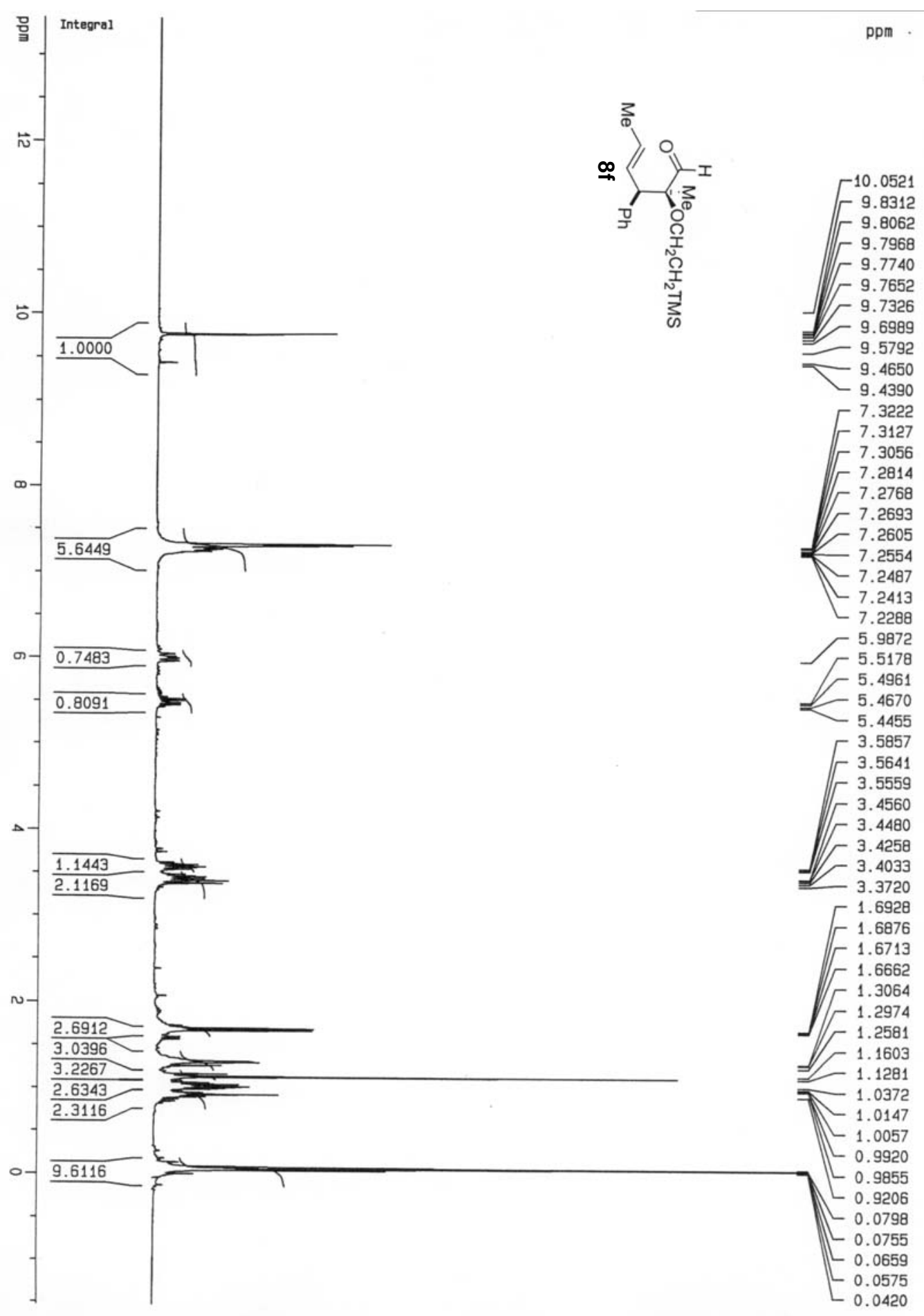



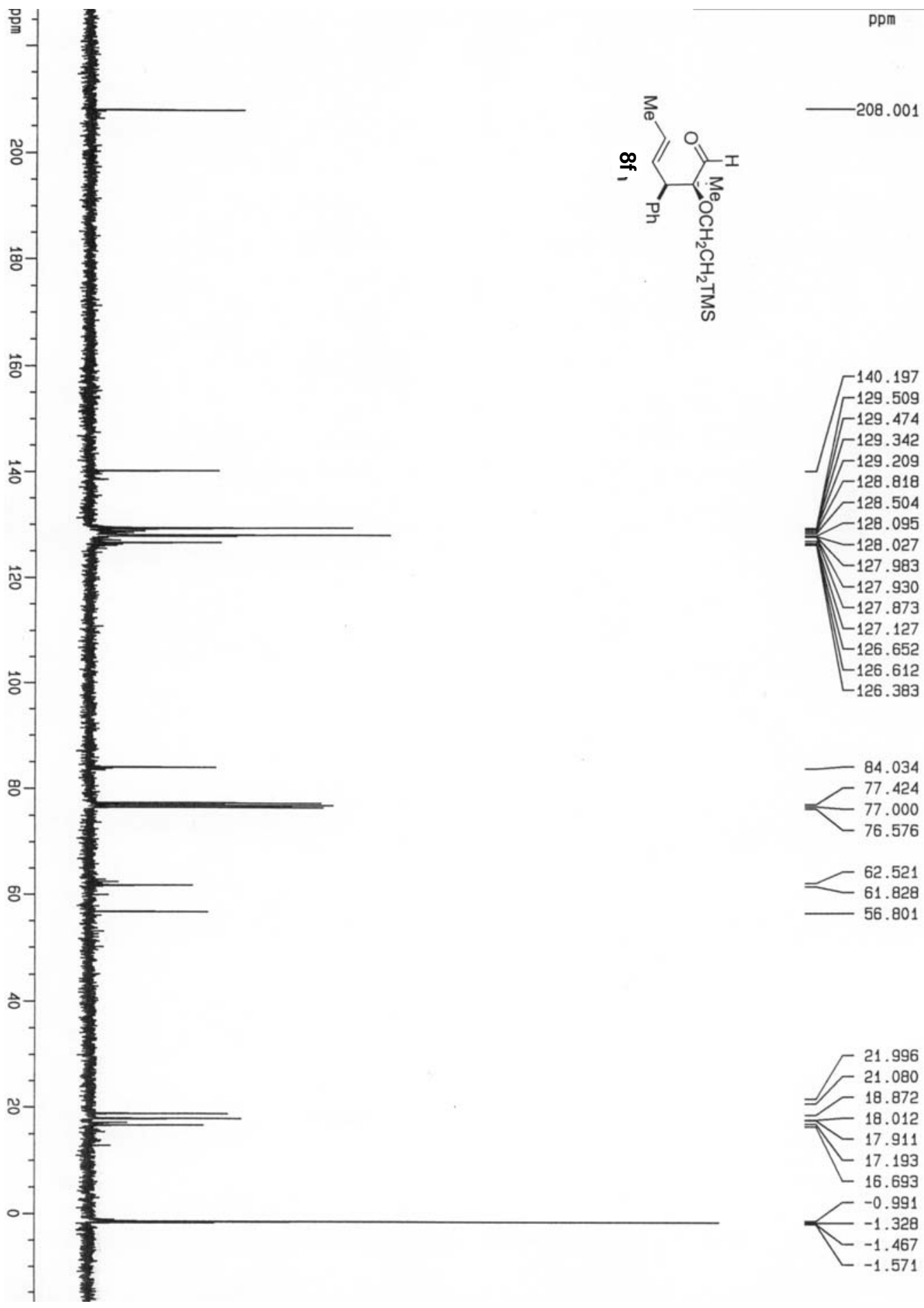


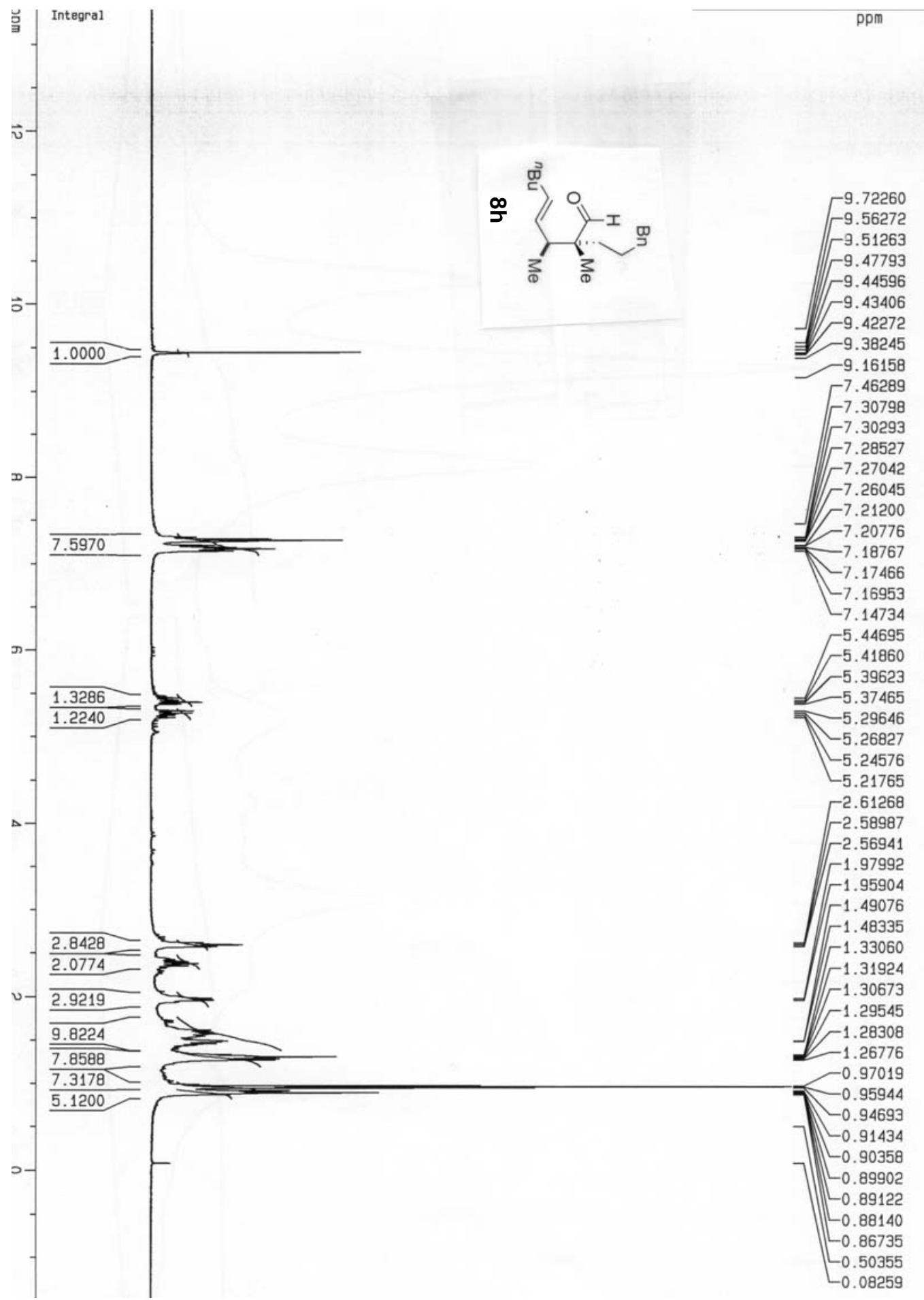




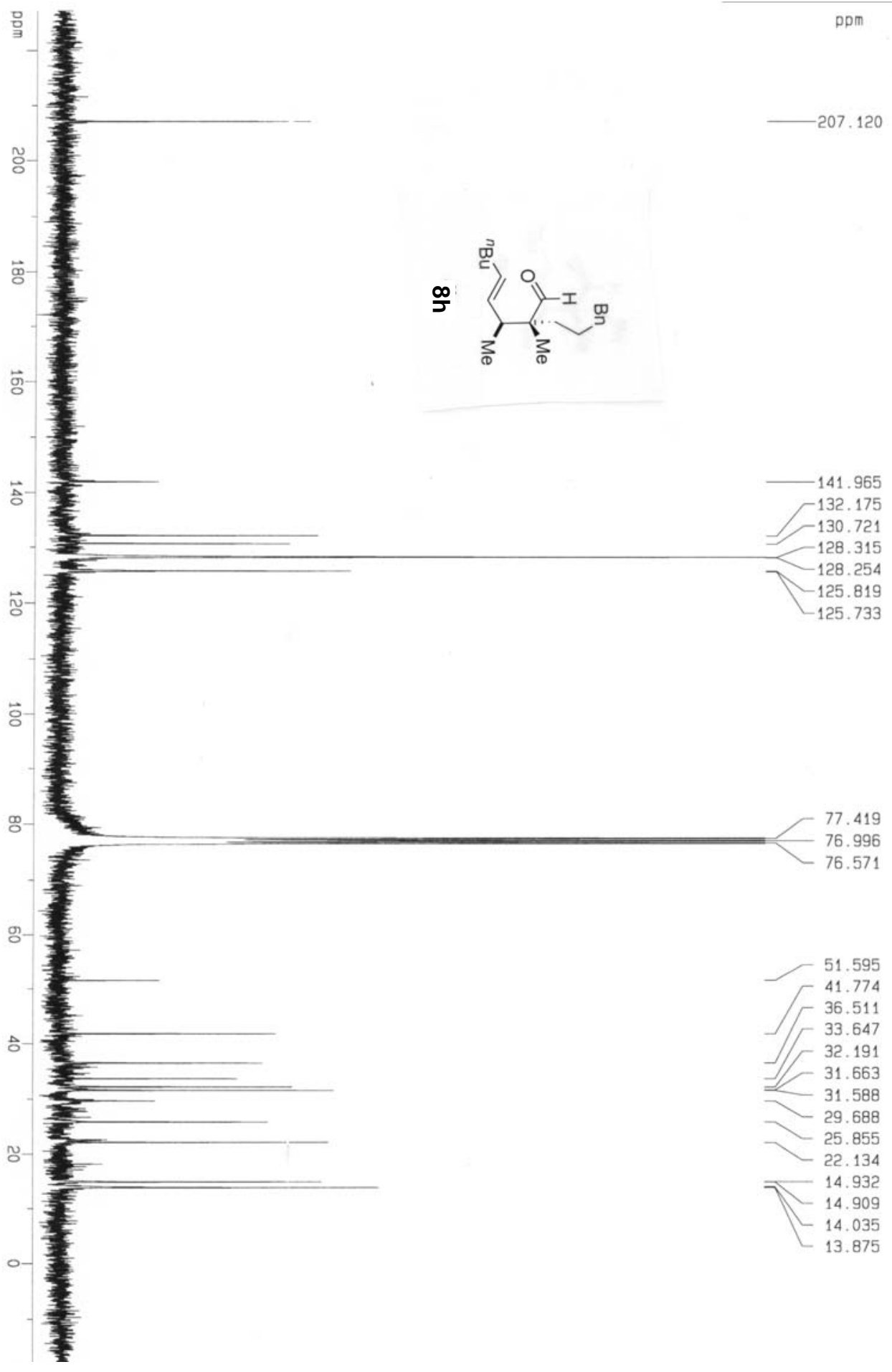




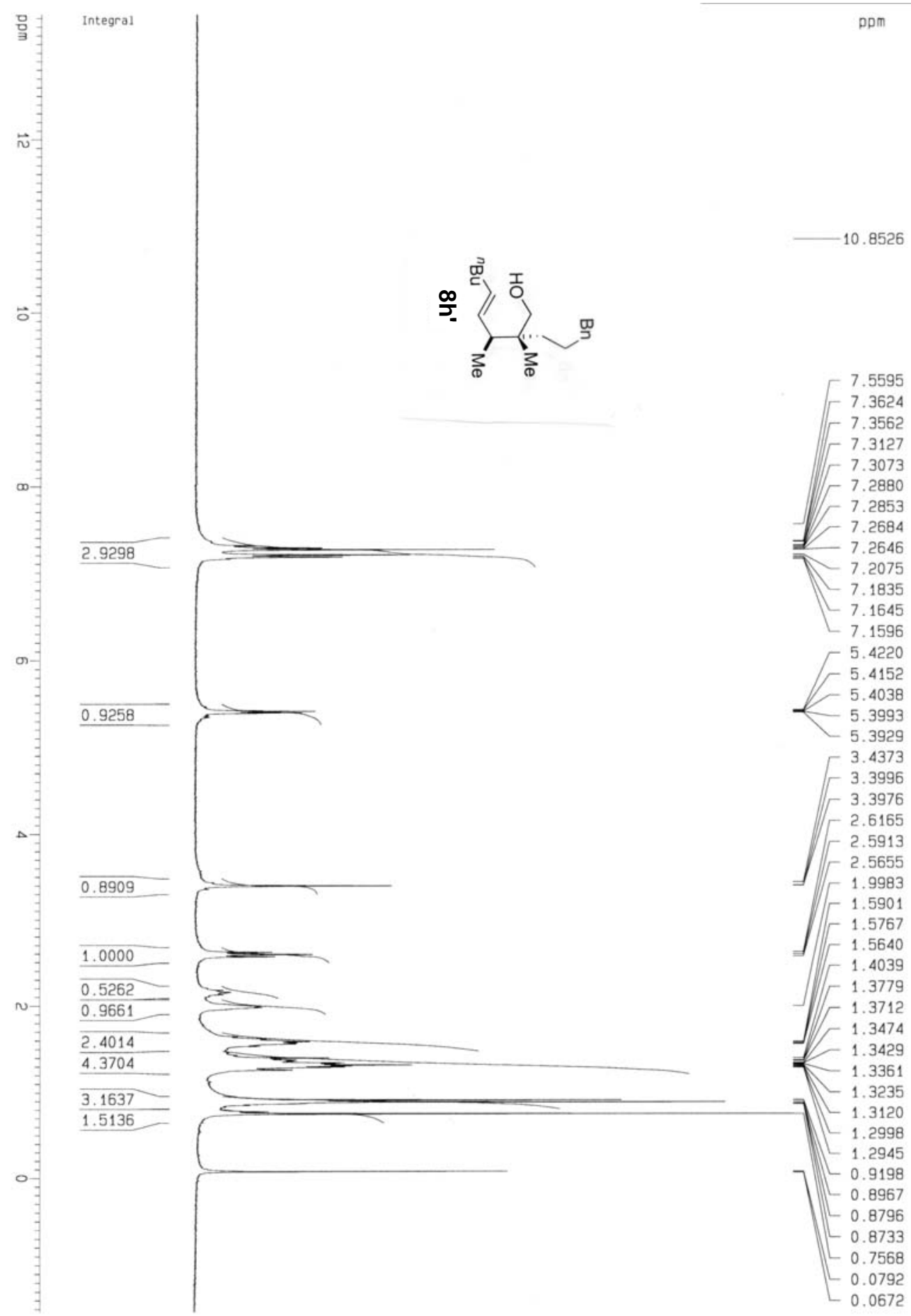




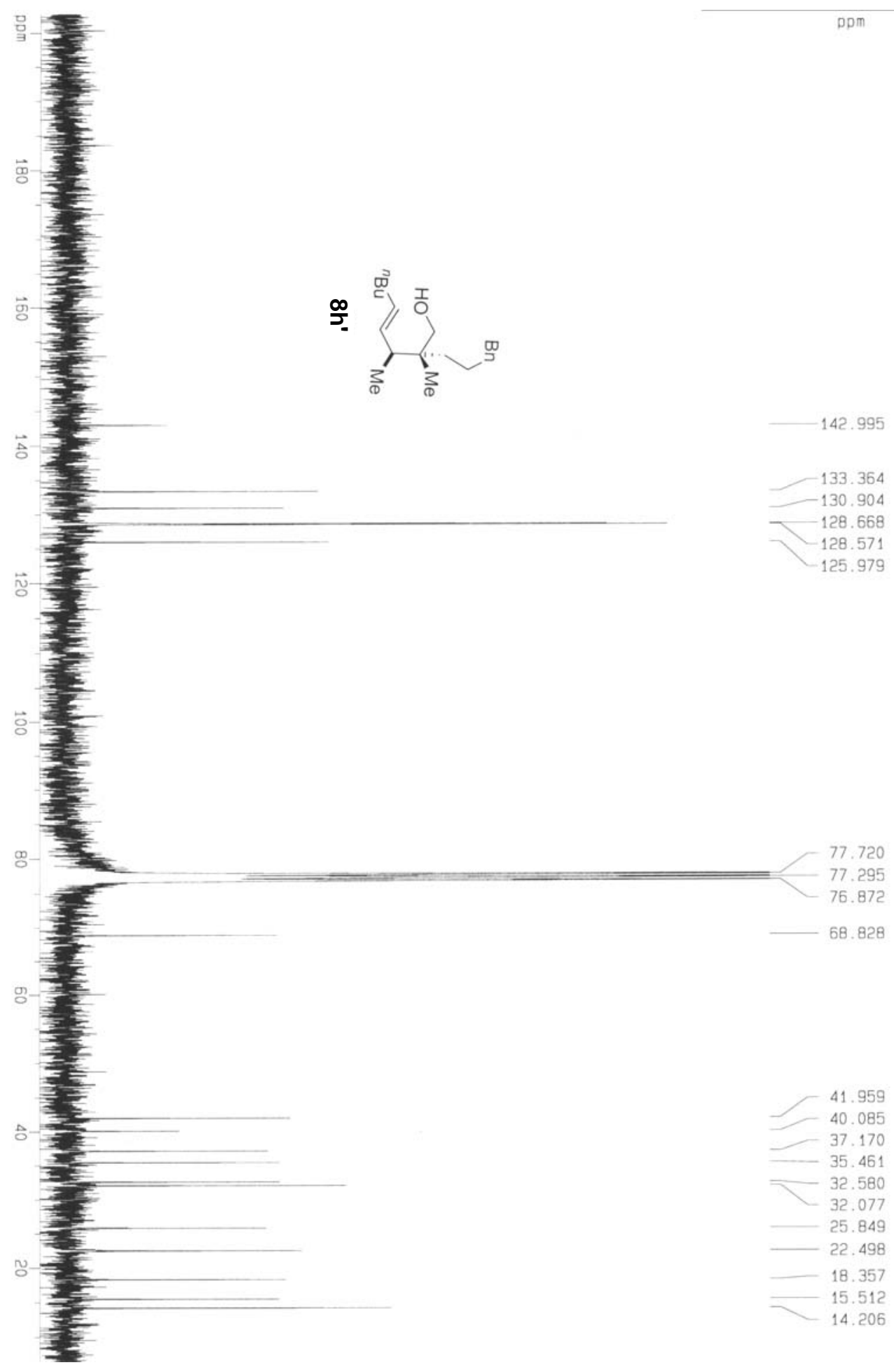




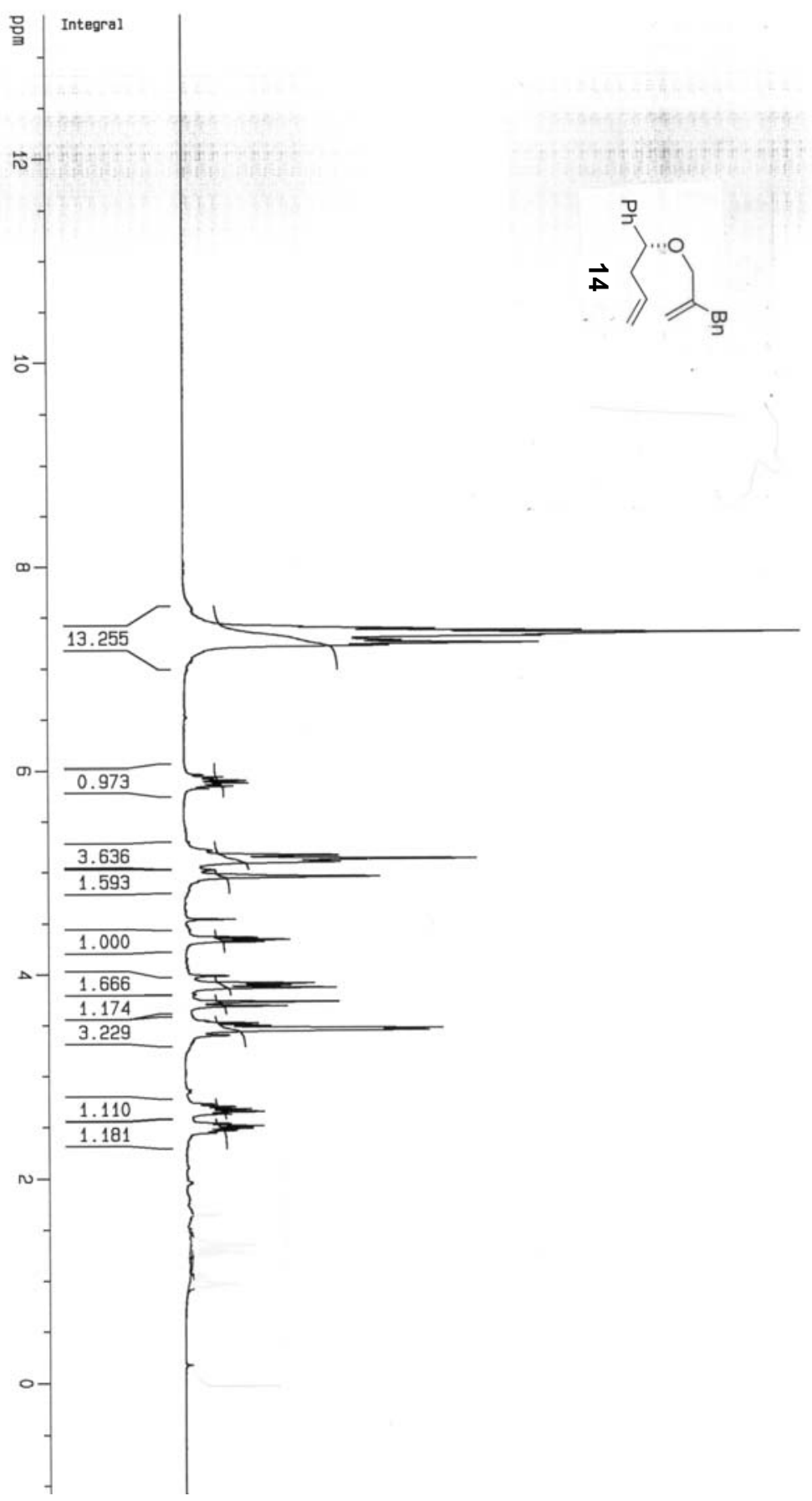




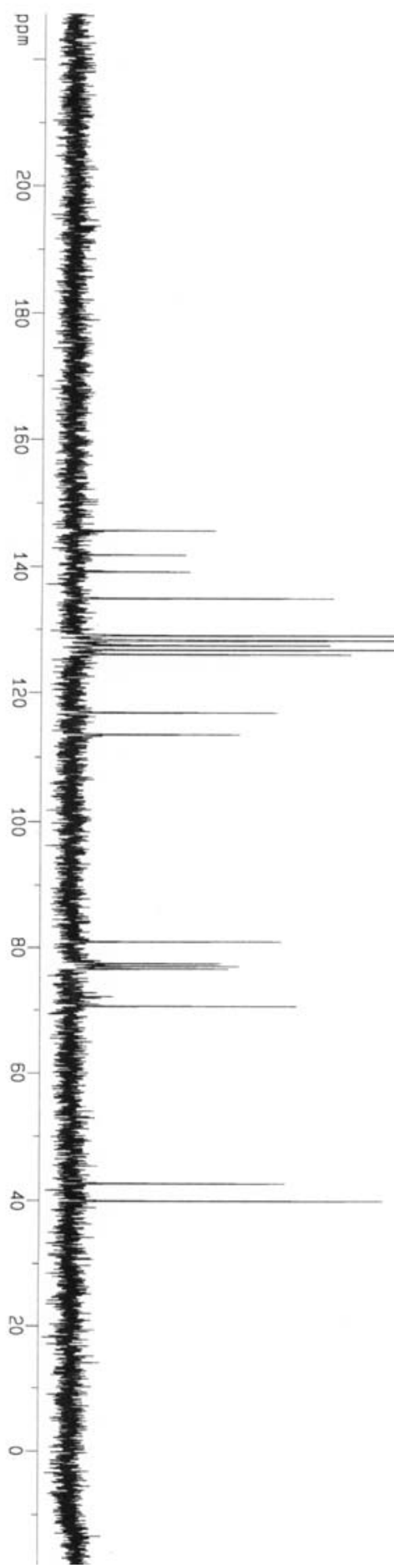

ppm
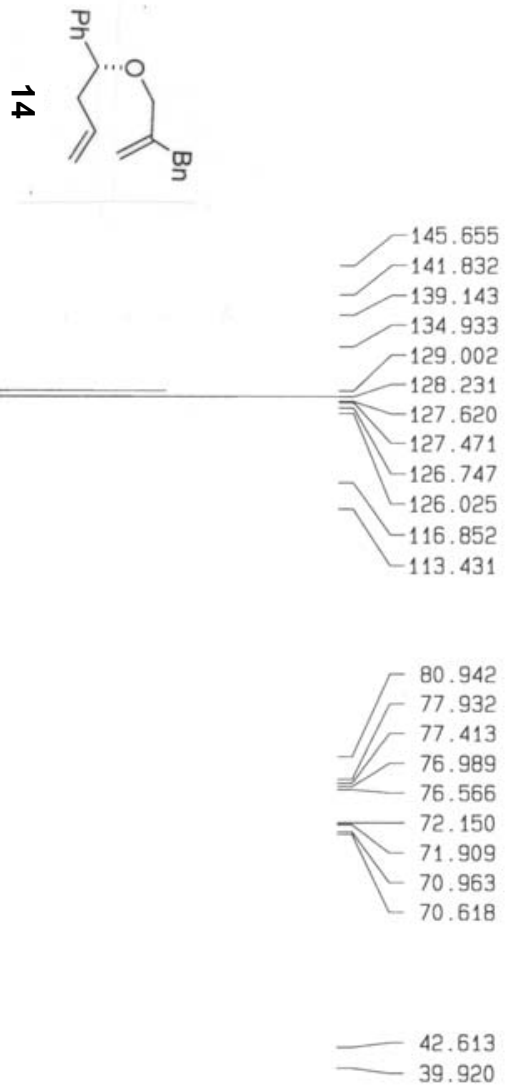

14.069

$-13.403$ 


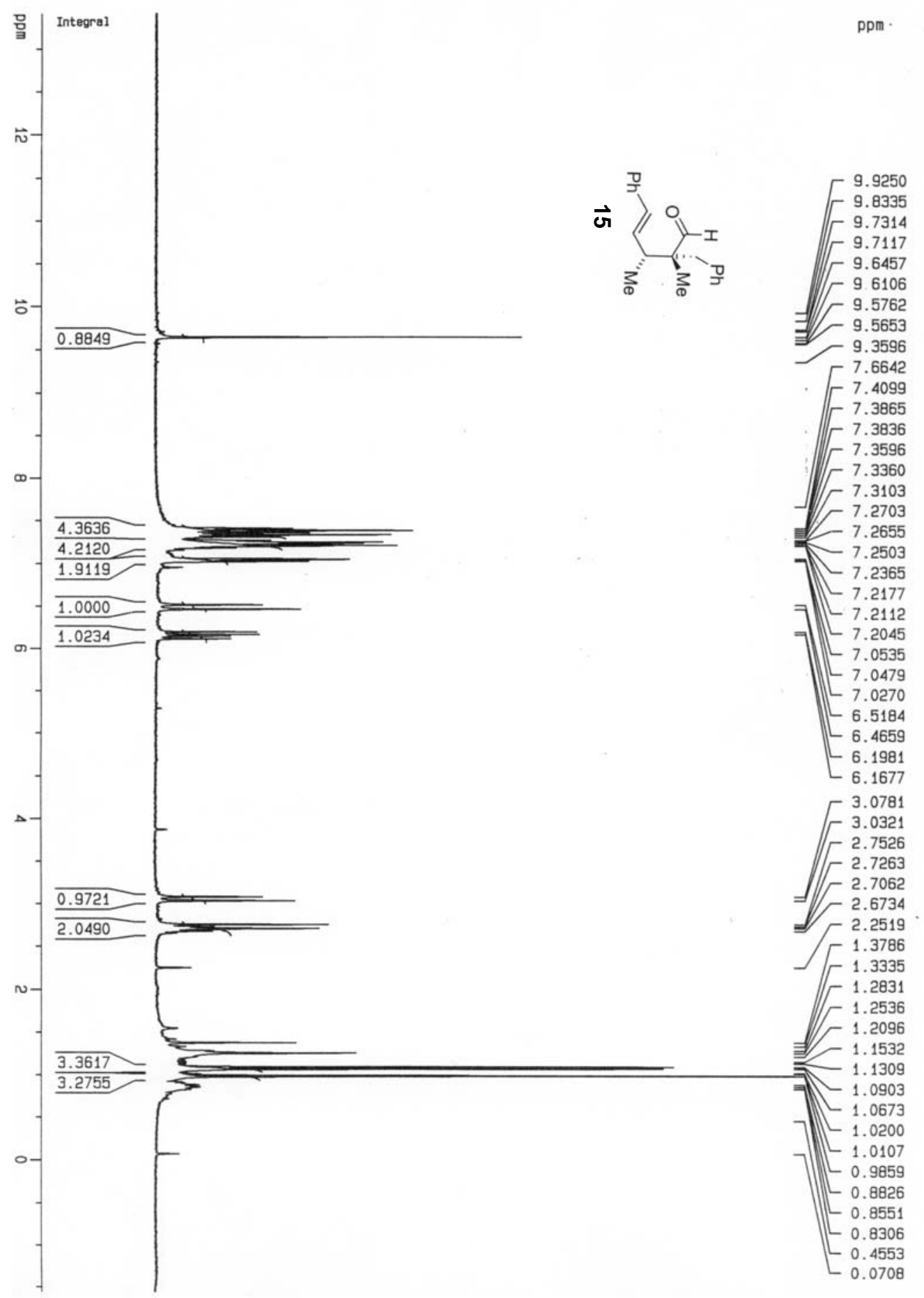




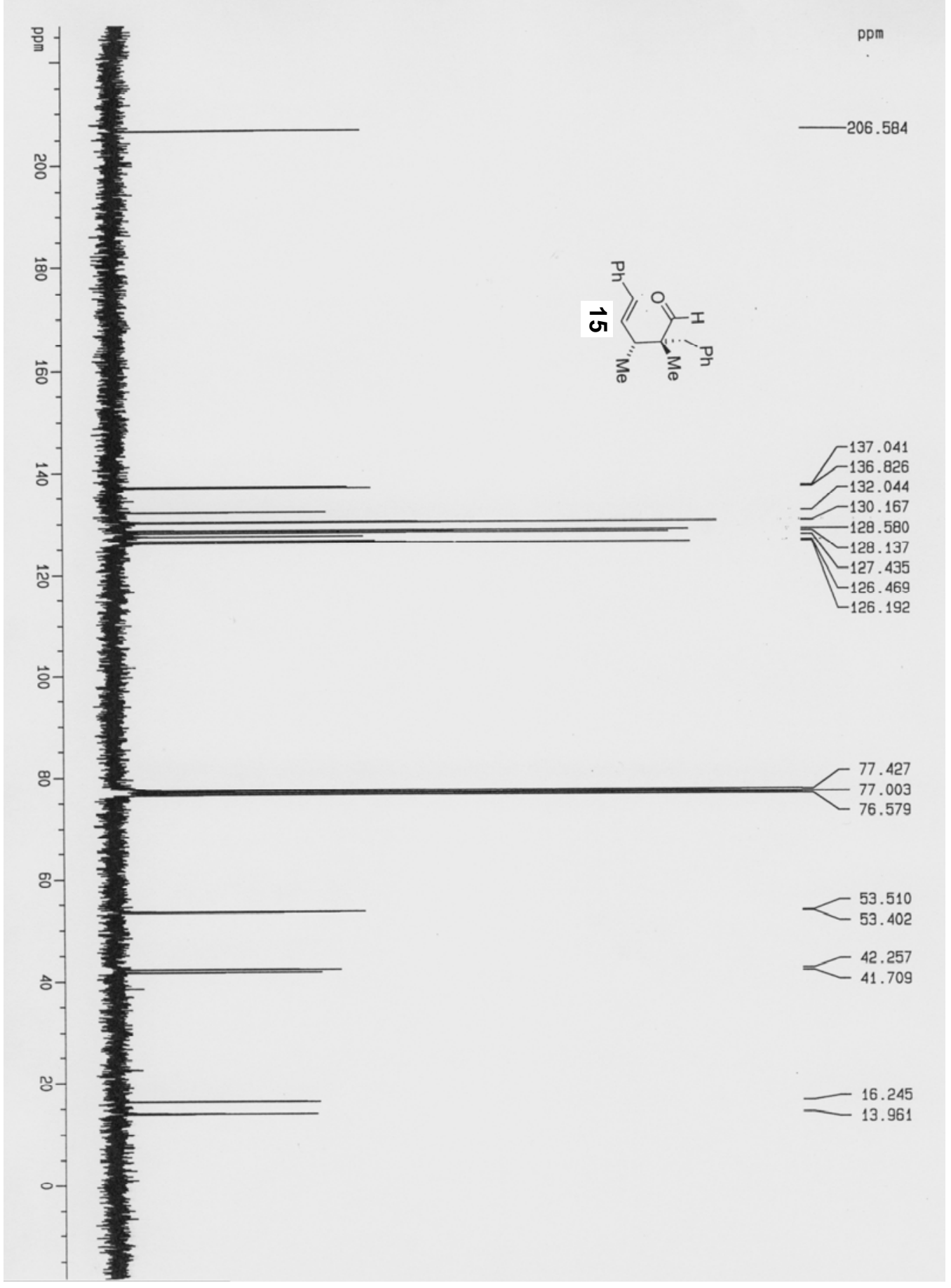



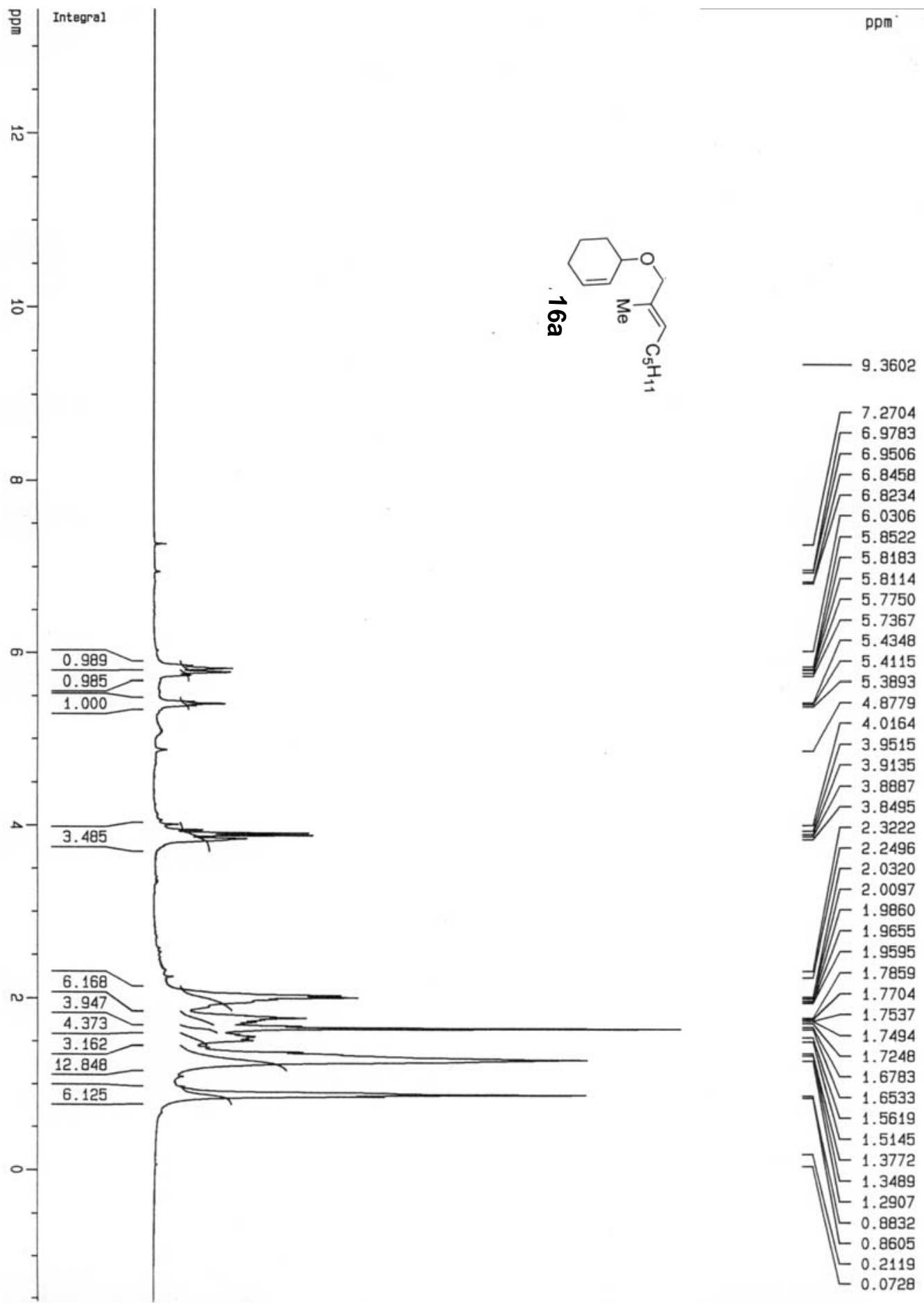


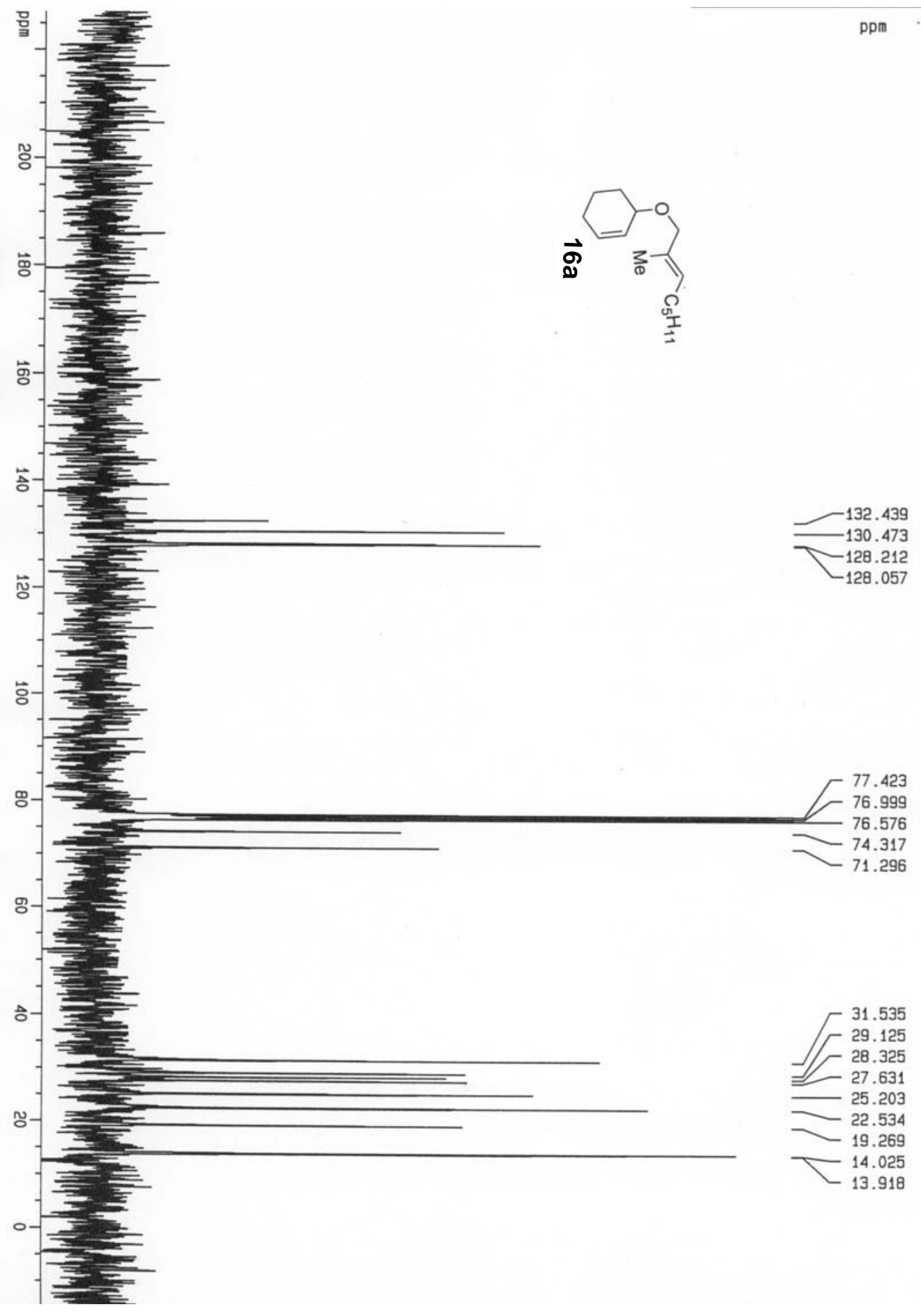




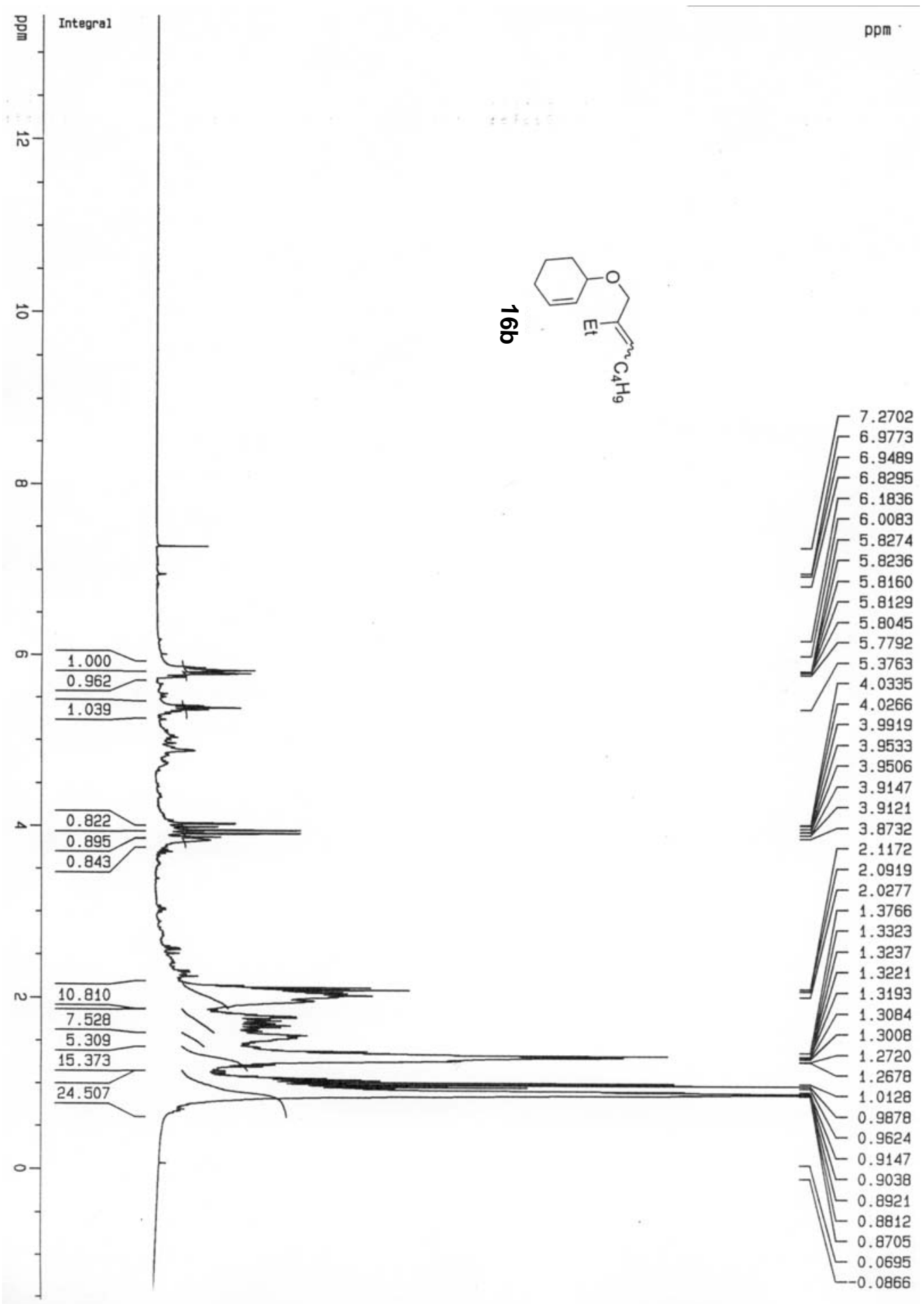




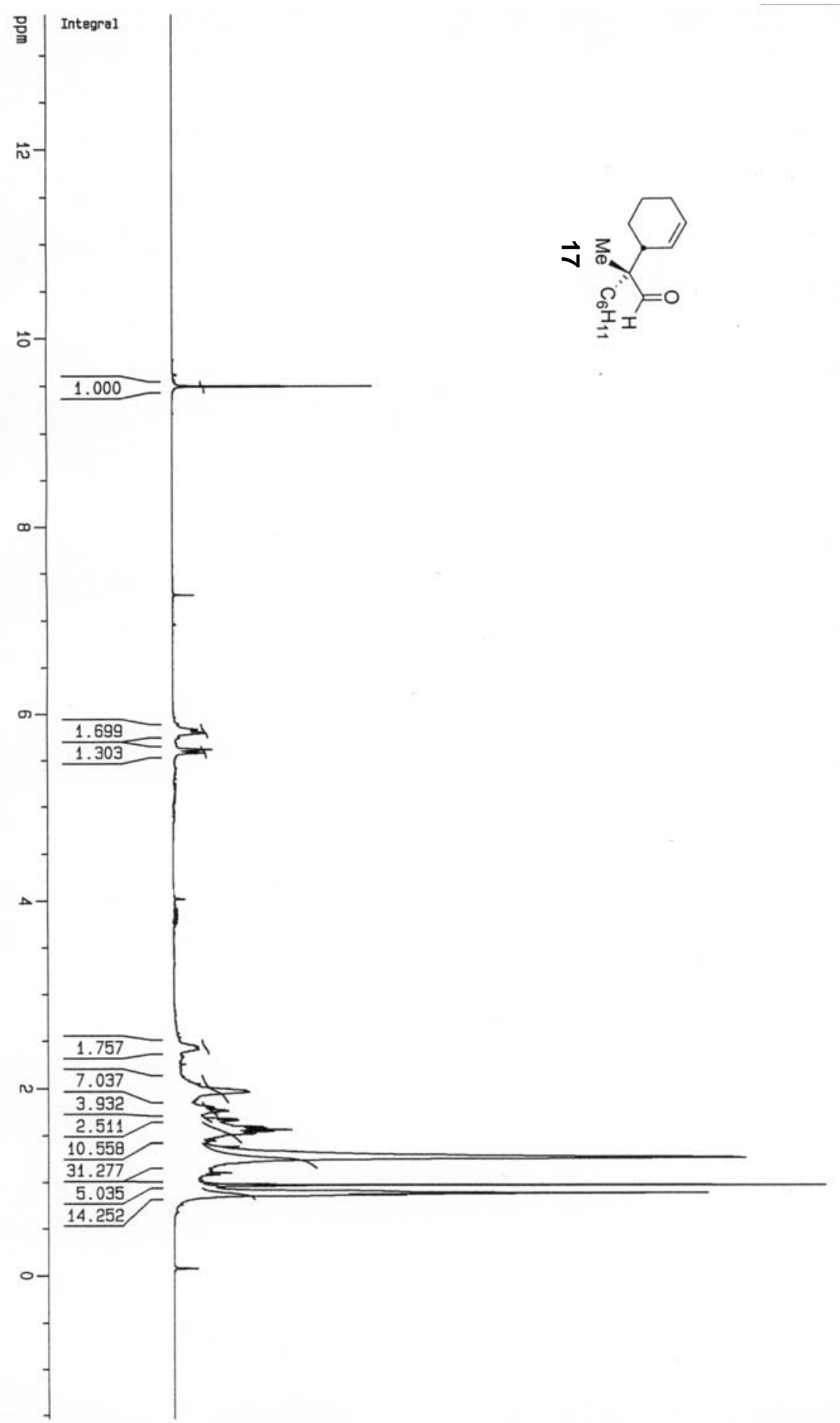

ppm

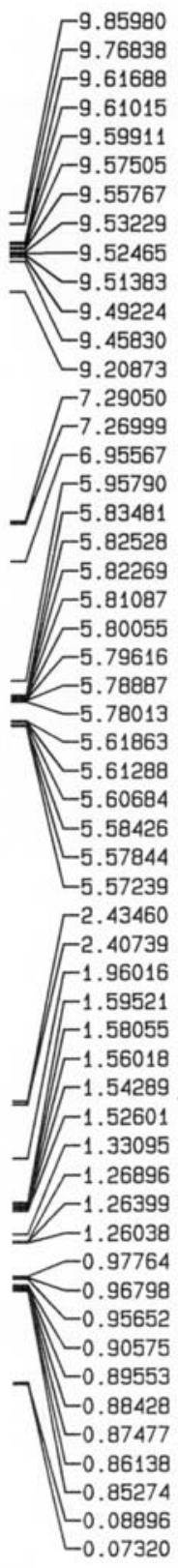




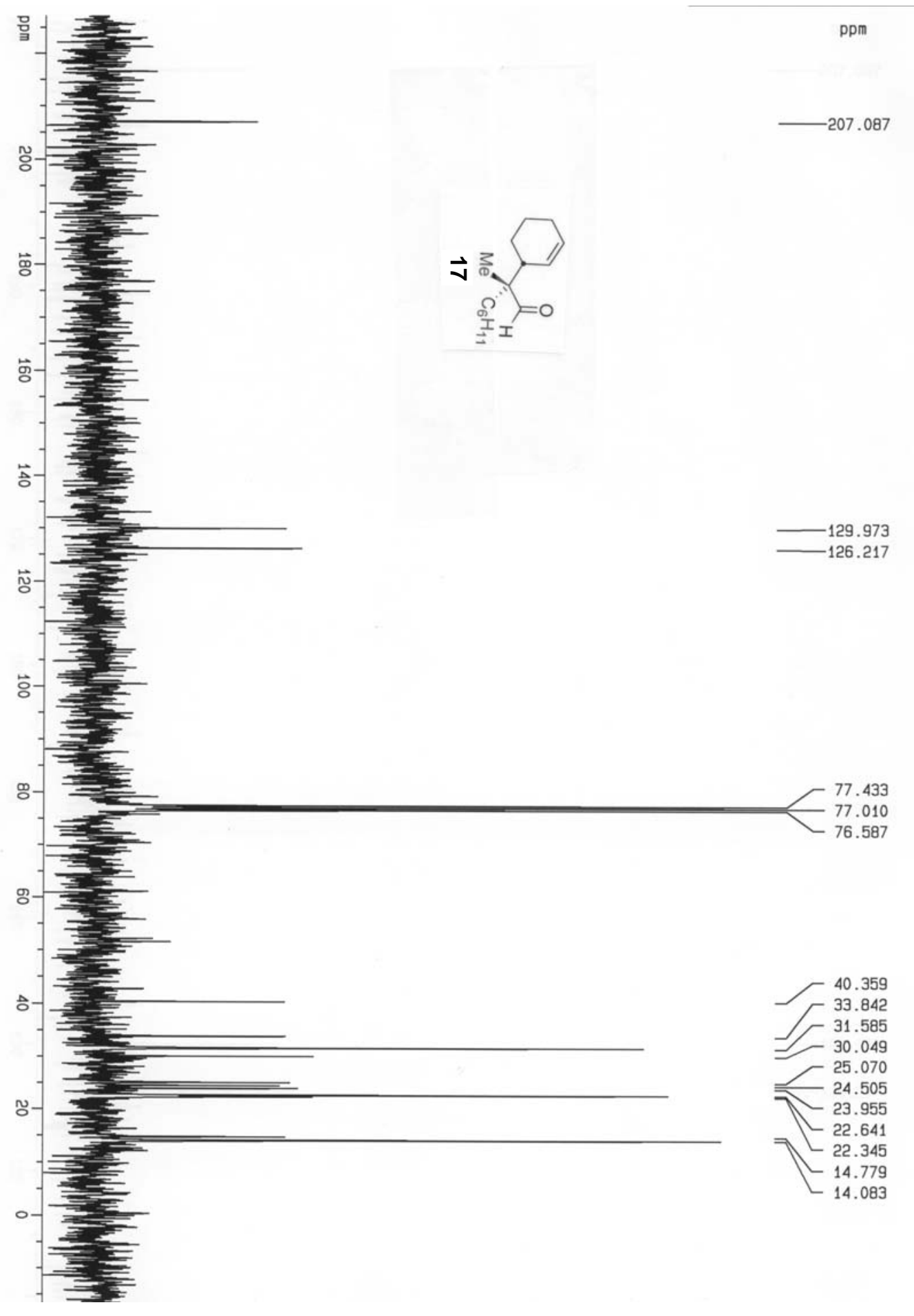




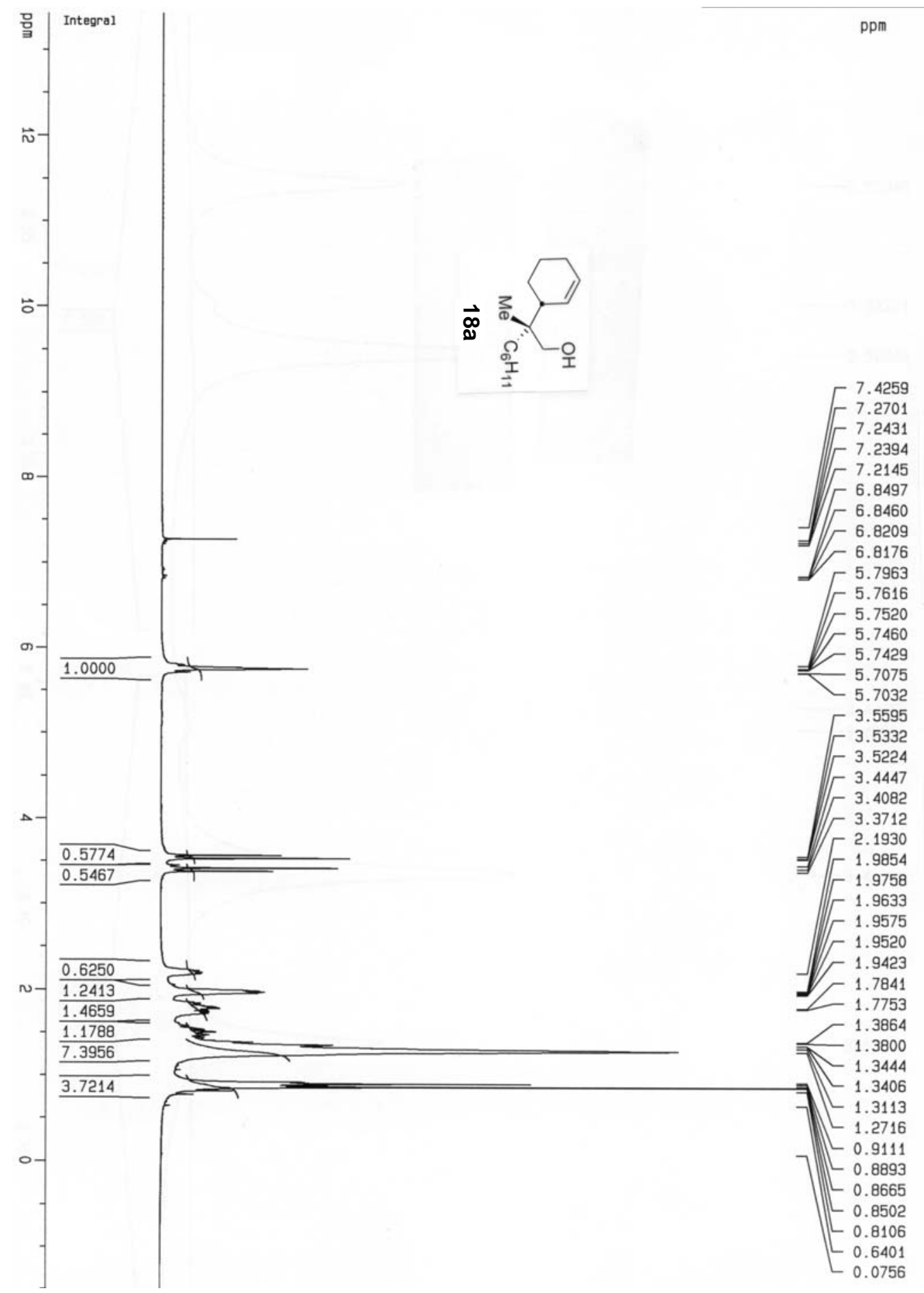




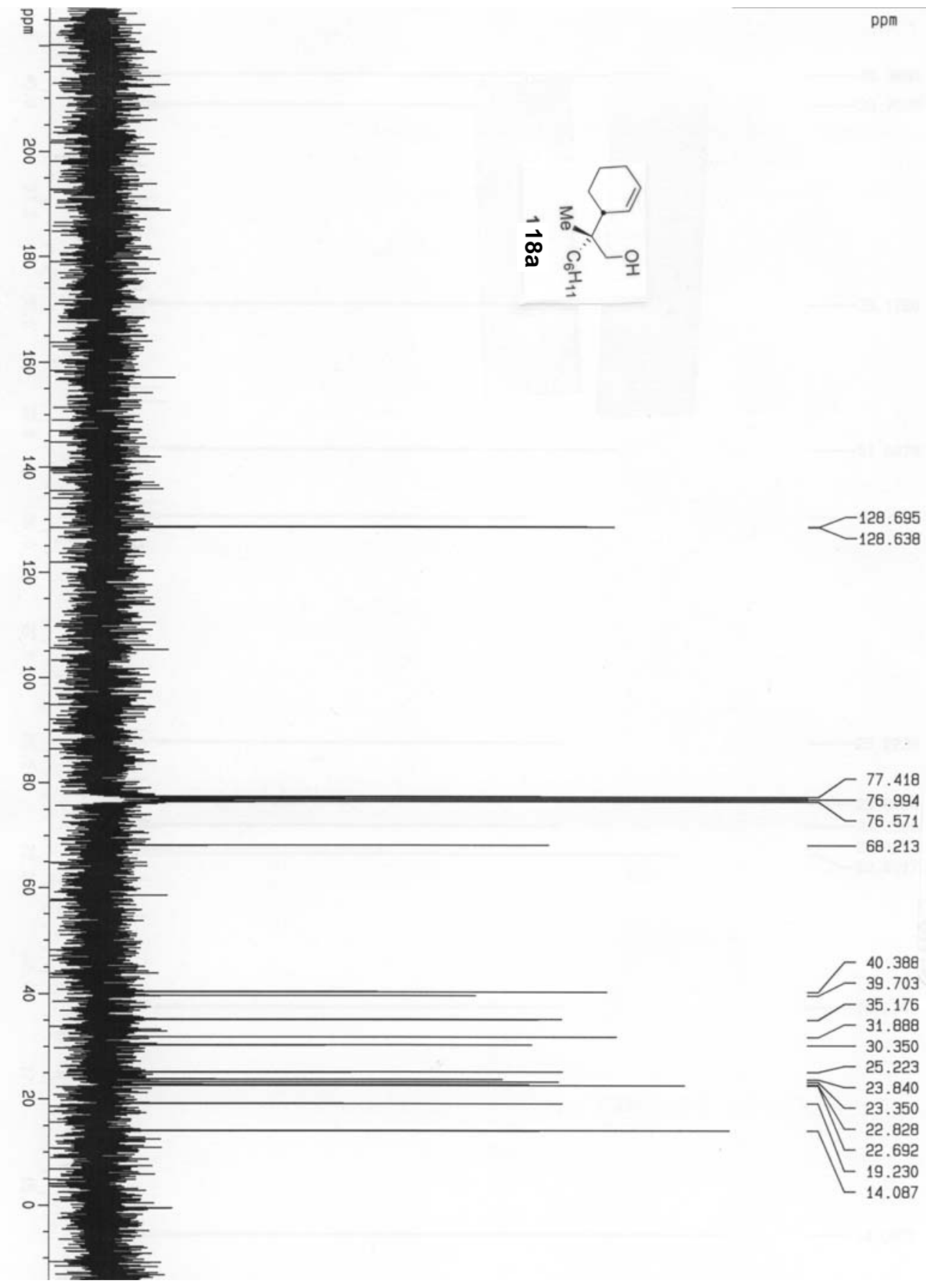




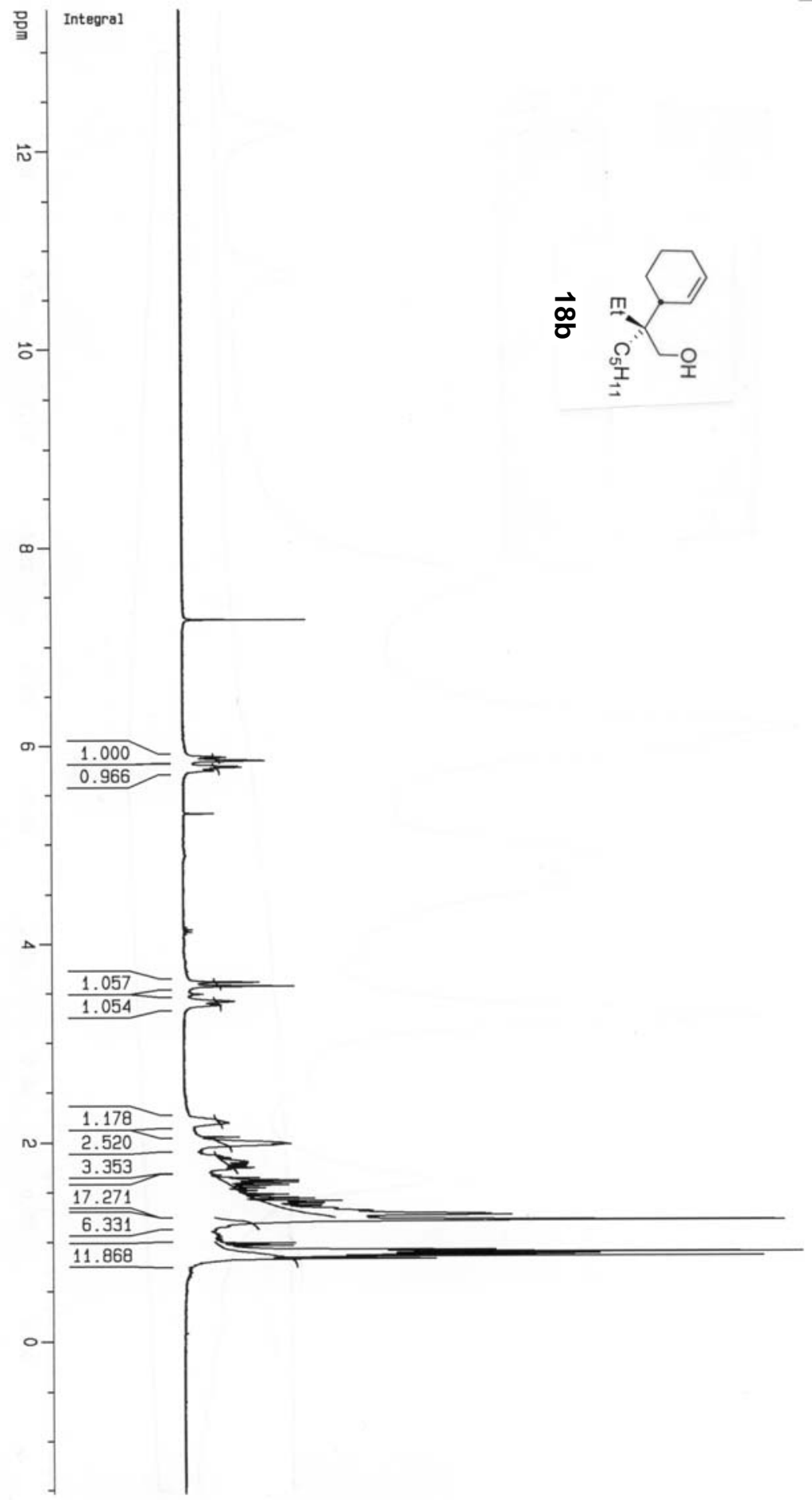

$-1.98739$

$-1.62007$

$-1.41240$

$-1.33574$

$-1.31209$

$-1.27536$

$-1.26599$

$-1.24627$

$-1.24238$

$-1.21128$

$-0.91644$

$\overbrace{0.89109}^{0.89367}$

$-0.88070$

0.87441

L 0.87020

L 0.85926

$-0.84959$

0.82456

-0.50484
-0.07653 


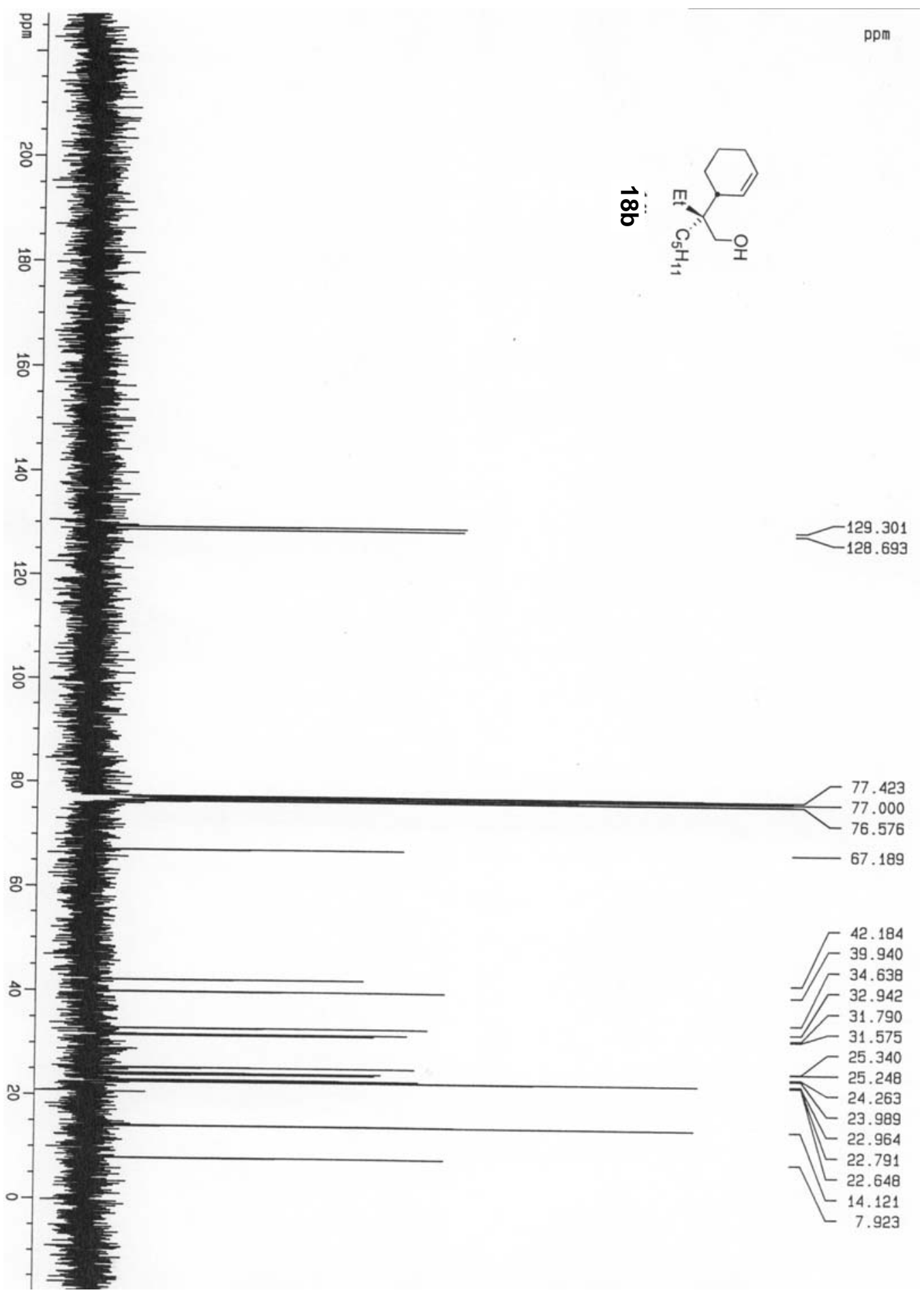

